\title{
Intramolecular, $\mathrm{Pd} / \mathrm{Cu}$-Cocatalyzed P-C Bond Cleavage and Addition onto an
} Alkyne: a Route to Benzophospholes

\author{
Yang Zhou ${ }^{a}$, Zhenjie Gan ${ }^{a}, \mathrm{Bo} \mathrm{Su}^{a}, \mathrm{Jun} \mathrm{Li}^{a}$, Zheng Duan* ${ }^{a}$, Francois Mathey* ${ }^{a, b}$ \\ a) College of Chemistry and Molecular Engineering, International Phosphorus Laboratory, \\ International Joint Research Laboratory for Functional Organophosphorus Materials of Henan \\ Province, Zhengzhou University, Zhengzhou 450001, P. R. China; \\ b) Division of Chemistry \& Biological Chemistry, Nanyang Technological University, 21 \\ Nanyang Link, Singapore 637371 \\ duanzheng@zzu.edu.cn; fmathey@ntu.edu.sg
}

Table of Contents

Page

General Experimental Details

S-2

General procedure and characterization data for 1

S-2

General procedure for the synthesis of benzophospholes

Synthesis of 4 .

S-4

General procedure for the synthesis of benzophosphole oxides.

S-5

Synthesis of $5 a$ and $5 b$

S-7

X-ray Crystallographic Studies of Compound 4

S-9

NMR date

S-13 


\section{Experimental Section}

Measurements. ${ }^{1} \mathrm{H},{ }^{13} \mathrm{C}$ and ${ }^{31} \mathrm{P}$ NMR spectra were recorded on Bruker $300 \mathrm{M}$ spectrometer. Chemical shifts are expressed in ppm from internal TMS $\left({ }^{1} \mathrm{H}\right.$ and $\left.{ }^{13} \mathrm{C}\right)$. All coupling constants ( $J$ values) are reported in Hertz $(\mathrm{Hz})$. HRMS were obtained on an Agilent 1290-6540 Q-Tof spectrometer by electrospray ionization (ESI). Element analytic data were obtained on a Thermo Electron Corporation flash EA 1112 element spectrometer. Melting points (uncorrected) were obtained on an X-4 micro-melting point apparatus. X-ray crystallographic analyses were performed on an Oxford diffraction Gemini E diffractometer.

Materials. All air- and moisture-sensitive manipulations were carried out routinely performed under an inert atmosphere of nitrogen by using standard Schlenk techniques and dry deoxygenated solvents. Dry toluene was obtained by distillation from $\mathrm{P}_{2} \mathrm{O}_{5}$ under atmosphere of dry nitrogen. Dry THF was distilled over sodium benzophenone ketyl under atmosphere of dry nitrogen. $\mathrm{Pd}\left(\mathrm{PPh}_{3}\right)_{2} \mathrm{Cl}_{2}$ was synthesized following the literature procedures. ${ }^{1}$ Ortho-bromophenylalkynes were synthesized by Sonogashira coupling ${ }^{2} . n$-butyl lithium (1.6 M in hexane) was purchased from Alfa Aesar, $\mathrm{Pd}(\mathrm{OAc})_{2}$ was purchased from Puyang Huicheng Electronic Material Co. Ltd. $\mathrm{CuI}$, and $\mathrm{CuBr}$ were purchased from Aladdin. Silica gel (200-300 mesh) purchased from Qingdao Hai Yang Chemical Industry Co. Ltd. was used for chromatographic separations. Other chemicals and solvents were purchased from commercial company and used as received.

\section{General procedure and characterization data of all new compounds for 1.}

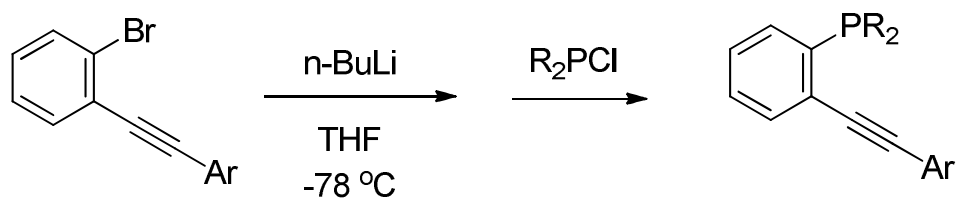

1a-1l were synthesized according to the literature procedures ${ }^{3}$. To a solution of alkyne $(5 \mathrm{mmol})$ in THF $(15 \mathrm{~mL})$ was added dropwise $n$-butyllithium in $n$-hexane $(3.4 \mathrm{~mL}$, $1.6 \mathrm{~mol} / \mathrm{L}, 5.5 \mathrm{mmol})$ at $-78{ }^{\circ} \mathrm{C}$ over $5 \mathrm{~min}$. under $\mathrm{N}_{2}$ atmosphere. The reaction mixture was stirred for $50 \mathrm{~min}$, then $\mathrm{R}_{2} \mathrm{PCl}(6 \mathrm{mmol})$ was added at $-78{ }^{\circ} \mathrm{C}$, then the temperature was slowly raised to room temperature and stirred for another $2 \mathrm{~h}$. After removal of the solvent under reduced pressure, the residue was chromatographed over silica gel to give pure products. For $\mathbf{1 k}$ and $\mathbf{1 l}$ because they are especially sensitive to oxygen, they were chromatographed over silica gel (dry deoxygenated $\mathrm{CH}_{2} \mathrm{Cl}_{2}$ as eluent) under atmosphere of dry nitrogen quickly. After evaporation of the solvent under reduced pressure, the crude product was obtained and used without further 
purification, and their structures were determined by the spectra of their oxides.

1d: white solid (1.03 g, $2.35 \mathrm{mmol}, 47 \%$ yield), ${ }^{1} \mathrm{H}$ NMR $\left(300 \mathrm{MHz}, \mathrm{CDCl}_{3}\right) \delta=$ 6.87-6.91 (m, 1H), 7.26-7.55 (m, 19H), 7.60-7.63 (m, 3H); ${ }^{13} \mathrm{C}$ NMR (75 MHz, $\left.\mathrm{CDCl}_{3}\right) \delta=89.38\left(\mathrm{~d}, J_{\mathrm{CP}}=7.2 \mathrm{~Hz}\right), 96.32\left(\mathrm{~d}, J_{\mathrm{CP}}=2.7 \mathrm{~Hz}\right), 121.96(\mathrm{C}), 126.82(2 \mathrm{CH})$, $127.02(2 \mathrm{CH}), 127.67(\mathrm{CH}), 128.00(\mathrm{C}), 128.32(2 \mathrm{CH}), 128.62\left(\mathrm{~d}, J_{\mathrm{CP}}=7.2 \mathrm{~Hz}, 4 \mathrm{CH}\right)$, $128.87(2 \mathrm{CH}), 128.89(2 \mathrm{CH}), 131.91(2 \mathrm{CH}), 132.29\left(\mathrm{~d}, J_{\mathrm{CP}}=3.6 \mathrm{~Hz}, \mathrm{CH}\right), 132.41$ $(\mathrm{CH}), 134.20\left(\mathrm{~d}, J_{\mathrm{CP}}=20.0 \mathrm{~Hz}, 4 \mathrm{CH}\right), 136.42\left(\mathrm{~d}, J_{\mathrm{CP}}=10.5 \mathrm{~Hz}, \mathrm{C}\right), 140.40(\mathrm{C})$, $140.77\left(\mathrm{~d}, J_{\mathrm{CP}}=12.5 \mathrm{~Hz}, \mathrm{C}\right), 140.97(\mathrm{C}) ;{ }^{31} \mathrm{P}\{1 \mathrm{H}\} \mathrm{NMR}\left(121 \mathrm{MHz}, \mathrm{CDCl}_{3}\right) \delta=-8.4$; HRMS Calcd. for $\mathrm{C}_{32} \mathrm{H}_{23} \mathrm{P}\left[\mathrm{M}+\mathrm{H}^{+}\right]$439.1610, Found: 439.1616 .

1f: white solid (1.14 g, $2.15 \mathrm{mmol}, 43 \%$ yield), ${ }^{1} \mathrm{H}$ NMR $\left(300 \mathrm{MHz}, \mathrm{CDCl}_{3}\right), \delta=$ 6.84-6.88 (m, 1H), 6.95-6.98 (m, 2H), 7.07-7.15 (m, 8H), 7.20-7.25 (m, 1H), 7.27-7.41 (m, 15H), 7.59-7.62 (m, 1H) ${ }^{13} \mathrm{C} \mathrm{NMR}(75 \mathrm{MHz}, \mathrm{CDCl} 3) \delta=88.04\left(\mathrm{~d}, J_{\mathrm{CP}}\right.$ $=7.4 \mathrm{~Hz}), 96.80\left(\mathrm{~d}, J_{\mathrm{CP}}=2.6 \mathrm{~Hz}\right), 115.97(\mathrm{C}), 122.21(2 \mathrm{CH}), 123.54(2 \mathrm{CH}), 124.99$ $(4 \mathrm{CH}), 127.98(\mathrm{CH}), 128.31(\mathrm{CH}), 128.38(\mathrm{C}), 128.59\left(\mathrm{~d}, J_{\mathrm{CP}}=7.1 \mathrm{~Hz}, 4 \mathrm{CH}\right), 128.84$ $(2 \mathrm{CH}), 129.43(4 \mathrm{CH}), 132.09\left(\mathrm{~d}, J_{\mathrm{CP}}=3.5 \mathrm{~Hz}, \mathrm{CH}\right), 132.39(\mathrm{CH}), 132.47(2 \mathrm{CH})$, $134.18\left(\mathrm{~d}, J_{\mathrm{CP}}=20.0 \mathrm{~Hz}, 4 \mathrm{CH}\right), 136.54\left(\mathrm{~d}, J_{\mathrm{CP}}=10.5 \mathrm{~Hz}, \mathrm{C}\right), 140.40\left(\mathrm{~d}, J_{\mathrm{CP}}=12.0 \mathrm{~Hz}\right.$, C), $147.25(\mathrm{C}), 147.95(\mathrm{C}) ;{ }^{31} \mathrm{P}\{1 \mathrm{H}\}$ NMR (121 MHz, $\left.\mathrm{CDCl}_{3}\right), \delta=-8.6$; HRMS Calcd. for $\mathrm{C}_{38} \mathrm{H}_{28} \mathrm{NP}\left[\mathrm{M}+\mathrm{H}^{+}\right]$530.2032, Found: 530.2033 .

1g: white solid (798 mg, $2.10 \mathrm{mmol}, 42 \%$ yield), ${ }^{1} \mathrm{H}$ NMR $\left(300 \mathrm{MHz}, \mathrm{CDCl}_{3}\right) \delta=$ 6.87-6.91 (m, 1H), $6.98(\mathrm{t}, J=8.7 \mathrm{~Hz}, 2 \mathrm{H}), 7.20-7.28(\mathrm{~m}, 3 \mathrm{H}), 7.32-7.43(\mathrm{~m}, 11 \mathrm{H})$, 7.60-7.64 (m, 1H); ${ }^{13} \mathrm{C}$ NMR $\left(75 \mathrm{MHz}, \mathrm{CDCl}_{3}\right) \delta=88.42\left(\mathrm{dd}, J_{1}=7.3 \mathrm{~Hz}, J_{2}=1.2\right.$ $\mathrm{Hz}), 95.36\left(\mathrm{~d}, J_{\mathrm{CP}}=2.7 \mathrm{~Hz}\right), 115.47\left(\mathrm{~d}, J_{\mathrm{CF}}=21.9 \mathrm{~Hz}, 2 \mathrm{CH}\right), 119.14\left(\mathrm{~d}, J_{\mathrm{CP}}=3.2 \mathrm{~Hz}\right.$, C), $127.59(\mathrm{~d}, J=28.2 \mathrm{~Hz}, \mathrm{C}), 128.37(\mathrm{CH}), 128.41(\mathrm{CH}), 128.65\left(\mathrm{~d}, J_{\mathrm{CP}}=7.3 \mathrm{~Hz}\right.$, $4 \mathrm{CH}), 128.95(2 \mathrm{CH}), 132.22\left(\mathrm{~d}, J_{C P}=3.5 \mathrm{~Hz}, \mathrm{CH}\right), 132.43(\mathrm{CH}), 133.38\left(\mathrm{~d}, J_{\mathrm{CF}}=8.3\right.$ $\mathrm{Hz}, 2 \mathrm{CH}), 134.18$ (d, $\left.J_{C P}=20.0 \mathrm{~Hz}, 4 \mathrm{CH}\right), 136.35$ (d, $\left.J=10.3 \mathrm{~Hz}, \mathrm{C}\right), 140.75$ (d, $J=$ $12.5 \mathrm{~Hz}, \mathrm{C}), 162.56\left(\mathrm{~d}, J_{\mathrm{CF}}=248.1 \mathrm{~Hz}, \mathrm{C}\right) ;{ }^{31} \mathrm{P}\{1 \mathrm{H}\} \mathrm{NMR}\left(121 \mathrm{MHz}, \mathrm{CDCl}_{3}\right) \delta=$ -8.3; HRMS Calcd. for $\mathrm{C}_{26} \mathrm{H}_{18} \mathrm{FP}\left[\mathrm{M}+\mathrm{H}^{+}\right]$381.1203, Found: 381.1209 .

1h: white solid (1.05 g, $2.44 \mathrm{mmol}, 49 \%$ yield), ${ }^{1} \mathrm{H}$ NMR $\left(300 \mathrm{MHz}, \mathrm{CDCl}_{3}\right) \delta=$ 6.86-6.91 (m, 1H), 7.25-7.74 (m, 14H), $7.53\left(\mathrm{~d}, J_{\mathrm{HH}}=8.1 \mathrm{~Hz}, 2 \mathrm{H}\right), 7.61-7.65(\mathrm{~m}, 1 \mathrm{H})$; ${ }^{13} \mathrm{C} \mathrm{NMR}\left(75 \mathrm{MHz}, \mathrm{CDCl}_{3}\right) \delta=90.96\left(\mathrm{~d}, J_{\mathrm{CP}}=7.2 \mathrm{~Hz}\right), 94.75,123.96\left(\mathrm{q}, J_{\mathrm{CF}}=270.5\right.$ $\mathrm{Hz}, \mathrm{C}), 125.05\left(\mathrm{q}, J_{\mathrm{CP}}=3.8 \mathrm{~Hz}, 2 \mathrm{CH}\right), 126.83(\mathrm{C}), 126.96\left(\mathrm{~d}, J_{\mathrm{CP}}=28.0 \mathrm{~Hz}, \mathrm{C}\right)$, $128.36(\mathrm{CH}), 128.66\left(\mathrm{~d}, J_{\mathrm{CP}}=7.3 \mathrm{~Hz}, 4 \mathrm{CH}\right), 128.86(\mathrm{CH}), 129.00(2 \mathrm{CH}), 129.84(\mathrm{q}$, $\left.J_{\mathrm{CF}}=32.4 \mathrm{~Hz}, \mathrm{C}\right), 131.62(2 \mathrm{CH}), 132.44\left(\mathrm{~d}, J_{\mathrm{CP}}=4.7 \mathrm{~Hz}, \mathrm{CH}\right), 132.47(\mathrm{CH}), 134.14$ $\left(\mathrm{d}, J_{\mathrm{CP}}=20.0 \mathrm{~Hz}, 4 \mathrm{CH}\right), 136.13\left(\mathrm{~d}, J_{\mathrm{CP}}=10.2 \mathrm{~Hz}, \mathrm{C}\right), 141.11\left(\mathrm{~d}, J_{\mathrm{CP}}=12.9 \mathrm{~Hz}, \mathrm{C}\right)$; ${ }^{31} \mathrm{P}\{1 \mathrm{H}\}$ NMR $\left(121 \mathrm{MHz}, \mathrm{CDCl}_{3}\right) \delta=-8.3$; HRMS Calcd. for $\mathrm{C}_{27} \mathrm{H}_{18} \mathrm{~F}_{3} \mathrm{P}\left[\mathrm{M}+\mathrm{H}^{+}\right]$ 431.1171, Found: 431.1179.

1k $P$-oxide: white solid (1.07 g, $3.16 \mathrm{mmol}, 63 \%$ yield), ${ }^{1} \mathrm{H}$ NMR (300 MHz, CDCl3) $\delta=0.90-0.97(\mathrm{~m}, 6 \mathrm{H}), 1.20-1.27(\mathrm{~m}, 6 \mathrm{H}), 2.60-2.74(\mathrm{~m}, 2 \mathrm{H}), 3.70(\mathrm{~s}, 3 \mathrm{H}), 6.81(\mathrm{~d}$, $\left.J_{\mathrm{HH}}=8.7 \mathrm{~Hz}, 2 \mathrm{H}\right), 7.27-7.35(\mathrm{~m}, 4 \mathrm{H}), 7.46-7.50(\mathrm{~m}, 1 \mathrm{H}), 7.80-8.08(\mathrm{~m}, 1 \mathrm{H}),{ }^{13} \mathrm{C}$ 
NMR $\left(75 \mathrm{MHz}, \mathrm{CDCl}_{3}\right) \delta=16.08\left(\mathrm{~d}, J_{\mathrm{CP}}=3.6 \mathrm{~Hz}, 2 \mathrm{CH}_{3}\right), 16.91\left(\mathrm{~d}, J_{\mathrm{CP}}=3.0 \mathrm{~Hz}\right.$, $\left.2 \mathrm{CH}_{3}\right), 26.71\left(\mathrm{~d}, J_{\mathrm{CP}}=65.9 \mathrm{~Hz}, 2 \mathrm{CH}\right), 55.24\left(\mathrm{CH}_{3}\right), 87.79\left(\mathrm{~d}, J_{\mathrm{CP}}=3.3 \mathrm{~Hz}\right), 94.34$, $114.35(2 \mathrm{CH}), 114.42(\mathrm{C}), 123.82\left(\mathrm{~d}, J_{\mathrm{CP}}=7.4 \mathrm{~Hz}, \mathrm{C}\right), 127.89\left(\mathrm{~d}, J_{\mathrm{CP}}=9.3 \mathrm{~Hz}, \mathrm{CH}\right)$, $130.86\left(\mathrm{~d}, J_{\mathrm{CP}}=2.1 \mathrm{~Hz}, \mathrm{CH}\right), 132.58(2 \mathrm{CH}), 133.03\left(\mathrm{~d}, J_{\mathrm{CP}}=80.6 \mathrm{~Hz}, \mathrm{C}\right), 133.31(\mathrm{~d}$, $\left.J_{\mathrm{PC}}=8.7 \mathrm{~Hz}, \mathrm{CH}\right), 134.51\left(\mathrm{~d}, J_{\mathrm{CP}}=4.8 \mathrm{~Hz}, \mathrm{CH}\right), 160.14(\mathrm{C}) ;{ }^{31} \mathrm{P}\{1 \mathrm{H}\}$ NMR $(121$ $\left.\mathrm{MHz}, \mathrm{CDCl}_{3}\right) \delta=51.2$; HRMS Calcd. for $\mathrm{C}_{21} \mathrm{H}_{25} \mathrm{O}_{2} \mathrm{P}\left[\mathrm{M}+\mathrm{H}^{+}\right]$341.1665, Found: 341.1674 .

11 -oxide: light yellow oil. (816 mg, $2.05 \mathrm{mmol}, 41 \%$ yield), ${ }^{1} \mathrm{H}$ NMR (300 MHz, $\left.\mathrm{CDCl}_{3}\right) \delta=1.05\left(\mathrm{t}, J_{\mathrm{HH}}=7.2 \mathrm{~Hz}, 12 \mathrm{H}\right), 3.06-3.22(\mathrm{~m}, 8 \mathrm{H}), 3.79(\mathrm{~s}, 3 \mathrm{H}), 6.82-6.87(\mathrm{~m}$, 2H), 7.28-7.42 (m, 2H), 7.47-7.52 (m, 2H), 7.56-7.61 (m, 1H), 7.76-7.83 (m, $1 \mathrm{H}) ;{ }^{13} \mathrm{C}$ NMR $\left(75 \mathrm{MHz} \mathrm{CDCl}_{3}\right) \delta=13.81\left(\mathrm{~d}, J_{\mathrm{CP}}=2.5 \mathrm{~Hz}, 4 \mathrm{CH}_{3}\right), 38.74\left(\mathrm{~d}, J_{\mathrm{CP}}=4.7 \mathrm{~Hz}\right.$, $\left.4 \mathrm{CH}_{2}\right), 55.25\left(\mathrm{CH}_{3}\right), 88.13\left(\mathrm{~d}, J_{\mathrm{CP}}=5.4 \mathrm{~Hz}\right), 94.41,113.93(2 \mathrm{CH}), 115.59(\mathrm{C}), 126.70$ $\left(\mathrm{d}, J_{\mathrm{CP}}=6.5 \mathrm{~Hz}, \mathrm{C}\right), 127.30\left(\mathrm{~d}, J_{\mathrm{CP}}=12.7 \mathrm{~Hz}, \mathrm{CH}\right), 130.51\left(\mathrm{~d}, J_{\mathrm{CP}}=2.6 \mathrm{~Hz}, \mathrm{CH}\right)$, $133.00(2 \mathrm{CH}), 133.48\left(\mathrm{~d}, J_{\mathrm{CP}}=8.7 \mathrm{~Hz}, \mathrm{CH}\right), 134.14\left(\mathrm{~d}, J_{\mathrm{CP}}=11.0 \mathrm{~Hz}, \mathrm{CH}\right), 134.41(\mathrm{~d}$, $\left.J_{\mathrm{CP}}=152.3 \mathrm{~Hz}, \mathrm{C}\right), 159.66(\mathrm{C}) ;{ }^{31} \mathrm{P}\{1 \mathrm{H}\} \mathrm{NMR}\left(121 \mathrm{MHz}, \mathrm{CDCl}_{3}\right) \delta=27.9$; HRMS Calcd. for $\mathrm{C}_{23} \mathrm{H}_{31} \mathrm{~N}_{2} \mathrm{O}_{2} \mathrm{P}\left[\mathrm{M}+\mathrm{H}^{+}\right]$399.2196, Found: 399.2202.

\section{General procedure for the synthesis of benzophospholes.}

A reaction vessel was charged with $1(2.0 \mathrm{mmol}), \mathrm{Pd}(\mathrm{OAc})_{2}(45 \mathrm{mg}, 0.20 \mathrm{mmol})$ or $\mathrm{Pd}\left(\mathrm{PPh}_{3}\right)_{2} \mathrm{Cl}_{2}(140 \mathrm{mg}, 0.20 \mathrm{mmol})$, and $\mathrm{CuI}(38 \mathrm{mg}, 0.2 \mathrm{mmol})$ toluene $(10 \mathrm{~mL})$ under a nitrogen atmosphere. The vessel was heated at $120^{\circ} \mathrm{C}$ until total consumption of the starting material as monitored by ${ }^{31} \mathrm{P}$ NMR . The reaction mixture was evaporated in vacuo. The crude product was chromatographed with Petroleum ether: $\mathrm{CH}_{2} \mathrm{Cl}_{2}$ (10:1), the benzophosphole was not pure completely $\left({ }^{31} \mathrm{P}\right.$ NMR: $\delta=0$ 4 ppm in $\mathrm{CH}_{2} \mathrm{Cl}_{2}$ ).

\section{Synthesis of 4 .}

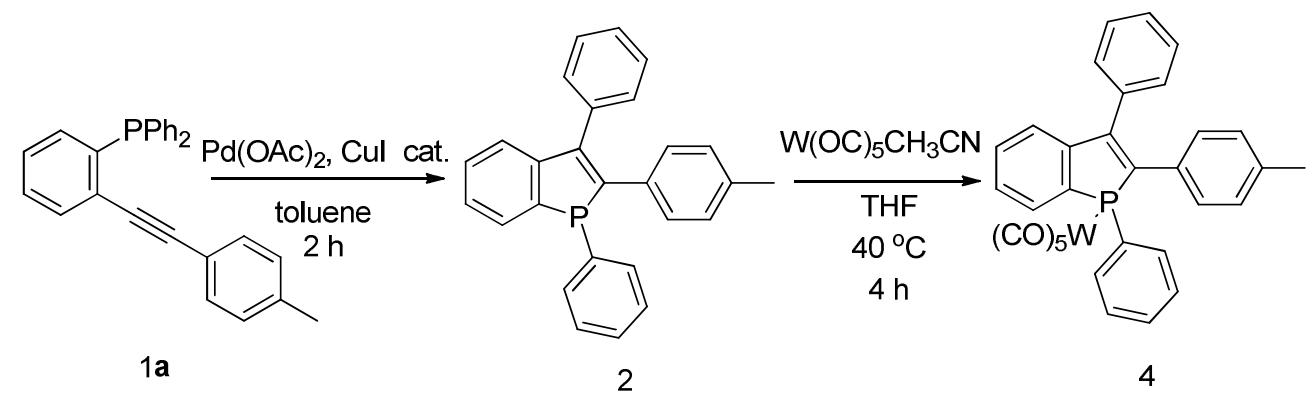

To a stirred solution of crude phosphole 2 (which was chromatographed with petroleum ether: $\left.\mathrm{CH}_{2} \mathrm{Cl}_{2}(10: 1)\right)(451 \mathrm{mg}, 1.20 \mathrm{mmol})$ in dry THF $(40 \mathrm{~mL})$, a freshly prepared THF $(20 \mathrm{~mL})$ solution of $\mathrm{W}(\mathrm{CO})_{5}\left(\mathrm{CH}_{3} \mathrm{CN}\right)(510 \mathrm{mg}, 1.40 \mathrm{mmol})$ was added. The reaction mixture was stirred at $40{ }^{\circ} \mathrm{C}$ for $4 \mathrm{~h}$. After evaporation of the solvent, the residue was chromatographed on silica gel with petroleum ether: $\mathrm{CH}_{2} \mathrm{Cl}_{2}$ (5:1) as eluent to give pure $\mathrm{P}-\mathrm{W}(\mathrm{CO})_{5}$ complex. 
4: light yellow solid (560 mg, $0.8 \mathrm{mmol}, 40 \%$ yield from 1a ); mp:199-201 ${ }^{\circ} \mathrm{C} ;{ }^{1} \mathrm{H}$ NMR $\left(300 \mathrm{MHz}, \mathrm{CDCl}_{3}\right) \delta=2.23(\mathrm{~s}, 3 \mathrm{H}), 6.77(\mathrm{~d}, J=8.1 \mathrm{~Hz}, 2 \mathrm{H}), 6.90(\mathrm{~d}, J=8.1 \mathrm{~Hz}$, $2 \mathrm{H}), 7.36-7.50(\mathrm{~m}, 10 \mathrm{H}), 7.61-7.71(\mathrm{~m}, 4 \mathrm{H}),{ }^{13} \mathrm{C} \mathrm{NMR}\left(75 \mathrm{MHz}, \mathrm{CDCl}_{3}\right) \delta=21.21$ $\left(\mathrm{CH}_{3}\right), 124.64\left(\mathrm{~d}, J_{\mathrm{CP}}=5.1 \mathrm{~Hz}, \mathrm{CH}\right), 127.86\left(\mathrm{~d}, J_{\mathrm{PC}}=9.6 \mathrm{~Hz}, \mathrm{CH}\right), 128.23(\mathrm{CH})$, $128.81(2 \mathrm{CH}), 128.89(2 \mathrm{CH}), 129.07\left(\mathrm{~d}, J_{\mathrm{CP}}=15.3 \mathrm{~Hz}, \mathrm{CH}\right), 129.24\left(\mathrm{~d}, J_{\mathrm{CP}}=10.3 \mathrm{~Hz}\right.$, $2 \mathrm{CH}), 129.61\left(\mathrm{~d}, J_{\mathrm{CP}}=5.5 \mathrm{~Hz}, 2 \mathrm{CH}\right), 129.75(2 \mathrm{CH}), 130.42(\mathrm{CH}), 131.22\left(\mathrm{~d}, J_{\mathrm{CP}}=\right.$ $12.6 \mathrm{~Hz}, \mathrm{C}), 131.28\left(\mathrm{~d}, J_{\mathrm{CP}}=34.3 \mathrm{~Hz}, \mathrm{C}\right), 131.31\left(\mathrm{~d}, J_{\mathrm{CP}}=2.1 \mathrm{~Hz}, \mathrm{CH}\right), 132.74\left(\mathrm{~d}, J_{\mathrm{CP}}\right.$ $=13.1 \mathrm{~Hz}, 2 \mathrm{CH}), 135.31\left(\mathrm{~d}, J_{\mathrm{CP}}=8.9 \mathrm{~Hz}, \mathrm{C}\right), 137.81(\mathrm{C}), 141.97\left(\mathrm{~d}, J_{\mathrm{CP}}=47.7 \mathrm{~Hz}, \mathrm{C}\right)$, $142.93\left(\mathrm{~d}, J_{\mathrm{CP}}=37.6 \mathrm{~Hz}, \mathrm{C}\right), 144.88\left(\mathrm{~d}, J_{\mathrm{CP}}=11.9 \mathrm{~Hz}, \mathrm{C}\right), 146.39\left(\mathrm{~d}, J_{\mathrm{CP}}=10.2 \mathrm{~Hz}, \mathrm{C}\right)$, $196.03\left(\mathrm{~d}, J_{\mathrm{CP}}=6.6 \mathrm{~Hz}\right.$, cis-CO), $198.14\left(\mathrm{~d}, J_{\mathrm{CP}}=20.6 \mathrm{~Hz}\right.$, trans $\left.-\mathrm{CO}\right) ;{ }^{31} \mathrm{P}\{1 \mathrm{H}\} \mathrm{NMR}$ $\left(121 \mathrm{MHz}, \mathrm{CDCl}_{3}\right) \delta=24.2\left(J_{\mathrm{PW}}=233 \mathrm{~Hz}\right)$; HRMS Calcd. for $\mathrm{C}_{32} \mathrm{H}_{21} \mathrm{O}_{5} \mathrm{PW}\left[\mathrm{M}+\mathrm{H}^{+}\right]$ 701.0709, Found: 701.0703; Anal. Calcd. for $\mathrm{C}_{32} \mathrm{H}_{21} \mathrm{O}_{5} \mathrm{PW}: \mathrm{C}, 54.88$; H, 3.02. Found: C, 54.51; H, 2.91.

\section{General procedure for the synthesis of benzophosphole oxides.}
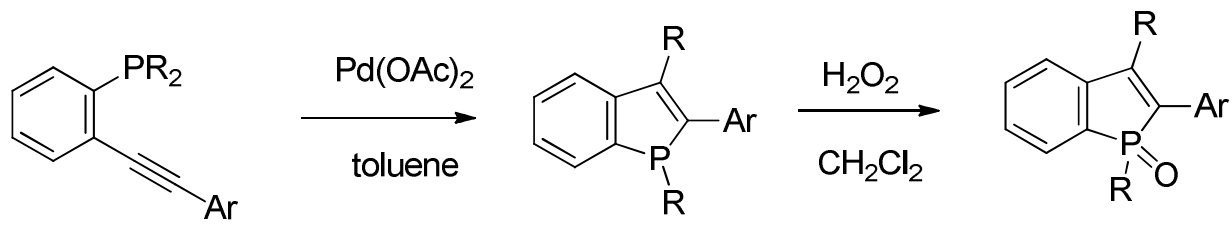

The benzophosphole 1a-f (which was chromatographed with Petroleum ether: $\mathrm{CH}_{2} \mathrm{Cl}_{2}$ (10:1)) was dissolved in $\mathrm{CH}_{2} \mathrm{Cl}_{2}(10 \mathrm{ml})$, was added an aqueous solution of $\mathrm{H}_{2} \mathrm{O}_{2}$ (ca. $30 \%$, a few drops), the reaction mixture was stirred at room temperature for $10 \mathrm{~min}$. The mixture was filtered through a short pad of silica gel with EtOAc, the filtrate was evaporated, and the residue was chromatographed on silica gel with $\mathrm{CH}_{2} \mathrm{Cl}_{2}$ :EtOAc $(5: 1)$ as eluent to give pure benzophosphole oxide.

3a: white solid (310 mg, $0.82 \mathrm{mmol}, 41 \%$ yield from 1a); ${ }^{1} \mathrm{H}$ NMR (300 MHz, $\left.\mathrm{CDCl}_{3}\right) \delta=7.08-7.11(\mathrm{~m}, 3 \mathrm{H}), 7.20-7.23(\mathrm{~m}, 3 \mathrm{H}), 7.32-7.49(\mathrm{~m}, 10 \mathrm{H}), 7.68-7.81(\mathrm{~m}$, $3 \mathrm{H}) ;{ }^{13} \mathrm{C}$ NMR $\left(75 \mathrm{MHz}, \mathrm{CDCl}_{3}\right) \delta=123.96\left(\mathrm{~d}, J_{\mathrm{CP}}=10.6 \mathrm{~Hz}, \mathrm{CH}\right), 127.79(\mathrm{CH})$, $128.16(2 \mathrm{CH}), 128.68(\mathrm{CH}), 128.72(2 \mathrm{CH}), 128.82(2 \mathrm{CH}), 128.82(\mathrm{CH}), 128.89$ $(2 \mathrm{CH}), 128.94(2 \mathrm{CH}), 129.09\left(\mathrm{~d}, J_{\mathrm{CP}}=10.5 \mathrm{~Hz}, \mathrm{CH}\right), 129.79\left(\mathrm{~d}, J_{\mathrm{CP}}=94.9 \mathrm{~Hz}, \mathrm{C}\right)$, $130.70\left(\mathrm{~d}, J_{\mathrm{CP}}=10.5 \mathrm{~Hz}, 2 \mathrm{CH}\right), 131.90\left(\mathrm{~d}, J_{\mathrm{CP}}=104.9 \mathrm{~Hz}, \mathrm{C}\right), 132.16(\mathrm{CH}), 132.61(\mathrm{~d}$, $\left.J_{\mathrm{CP}}=9.7 \mathrm{~Hz}, \mathrm{C}\right), 132.91(\mathrm{CH}), 133.95\left(\mathrm{~d}, J_{\mathrm{CP}}=14.7 \mathrm{~Hz}, \mathrm{C}\right), 134.09\left(\mathrm{~d}, J_{\mathrm{CP}}=94.8 \mathrm{~Hz}\right.$, C), $143.46\left(\mathrm{~d}, J_{\mathrm{CP}}=26.8 \mathrm{~Hz}, \mathrm{C}\right), 149.89\left(\mathrm{~d}, J_{\mathrm{CP}}=21 \mathrm{~Hz}, \mathrm{C}\right) ;{ }^{31} \mathrm{P}\{1 \mathrm{H}\} \mathrm{NMR}(121 \mathrm{MHz}$, $\left.\mathrm{CDCl}_{3}\right) \delta=38.7$; HRMS (ESI) Calcd for $\mathrm{C}_{26} \mathrm{H}_{20} \mathrm{OP}[\mathrm{M}+\mathrm{H}]+379.1246$, found 379.1248 .

3b: yellow solid (408 mg, $1.04 \mathrm{mmol}, 52 \%$ yield from 1b); mp:161-163 ${ }^{\circ} \mathrm{C} ;{ }^{1} \mathrm{H}$ NMR $\left(300 \mathrm{MHz}, \mathrm{CDCl}_{3}\right) \delta=2.07(\mathrm{~s}, 3 \mathrm{H}), 6.82\left(\mathrm{~d}, J_{\mathrm{HH}}=7.8 \mathrm{~Hz}, 2 \mathrm{H}\right), 7.14-7.17(\mathrm{~m}, 3 \mathrm{H})$, 7.28-7.36 (m, 10H), 7.60-7.66 (m, 1H), 7.73-7.79 (m, 2H); ${ }^{13} \mathrm{C}$ NMR $(75 \mathrm{MHz}$, 
$\left.\mathrm{CDCl}_{3}\right) \delta=21.20\left(\mathrm{CH}_{3}\right), 123.89\left(\mathrm{~d}, J_{\mathrm{CP}}=10.8 \mathrm{~Hz}, \mathrm{CH}\right), 128.65(\mathrm{CH}), 128.85(2 \mathrm{CH})$, $128.86\left(\mathrm{~d}, J_{\mathrm{CP}}=12 \mathrm{~Hz}, 2 \mathrm{CH}\right), 128.94(2 \mathrm{CH}), 128.98(2 \mathrm{CH}), 129.04$ (overlap, 4CH), $129.73\left(\mathrm{~d}, J_{\mathrm{CP}}=9.8 \mathrm{~Hz}, \mathrm{C}\right), 130.04\left(\mathrm{~d}, J_{\mathrm{CP}}=98.9 \mathrm{~Hz}, \mathrm{C}\right), 130.88\left(\mathrm{~d}, J_{\mathrm{PC}}=10.5 \mathrm{~Hz}\right.$, $2 \mathrm{CH}), 132.00\left(\mathrm{~d}, J_{\mathrm{CP}}=105.0 \mathrm{~Hz}, \mathrm{C}\right), 132.15\left(\mathrm{~d}, J_{\mathrm{CP}}=2.5 \mathrm{~Hz}, \mathrm{CH}\right), 132.92(\mathrm{CH})$, $134.07\left(\mathrm{~d}, J_{\mathrm{CP}}=95.1 \mathrm{~Hz}, \mathrm{C}\right), 134.43\left(\mathrm{~d}, J_{\mathrm{CP}}=15.1 \mathrm{~Hz}, \mathrm{C}\right), 137.76(\mathrm{C}), 143.84\left(\mathrm{~d}, J_{\mathrm{CP}}=\right.$ $26.9 \mathrm{~Hz}, \mathrm{C}), 149.26\left(\mathrm{~d}, J_{\mathrm{CP}}=21.5 \mathrm{~Hz}, \mathrm{C}\right) ;{ }^{31} \mathrm{P}\{1 \mathrm{H}\} \mathrm{NMR}\left(121 \mathrm{MHz}, \mathrm{CDCl}_{3}\right) \delta=39.2$; HRMS Calcd. for $\mathrm{C}_{27} \mathrm{H}_{21} \mathrm{OP}\left[\mathrm{M}+\mathrm{H}^{+}\right]$393.1403, Found: 393.1410; Anal. Calcd. for $\mathrm{C}_{27} \mathrm{H}_{21} \mathrm{OP}: \mathrm{C}, 82.64 ; \mathrm{H}, 5.39$. Found: C, 82.62; H, 5.60.

3c: colorless oil (373 mg, $0.86 \mathrm{mmol}, 43 \%$ yield from 1c), ${ }^{1} \mathrm{H}$ NMR (300 MHz, $\left.\mathrm{CDCl}_{3}\right) \delta=0.85(3 \mathrm{H}), 1.22(2 \mathrm{H}), 1.46(2 \mathrm{H}), 2.44(2 \mathrm{H}), 6.88-6.91\left(\mathrm{~d}, J_{\mathrm{HH}}=8.1 \mathrm{~Hz}\right.$, $2 \mathrm{H}), 7.145-7.17(\mathrm{~m}, 3 \mathrm{H}), 7.29-7.47(\mathrm{~m}, 10 \mathrm{H}), 7.65-7.71(\mathrm{~m}, 1 \mathrm{H}), 7.76-7.83(\mathrm{~m}, 2 \mathrm{H})$, ${ }^{13} \mathrm{C} \mathrm{NMR}\left(75 \mathrm{MHz}, \mathrm{CDCl}_{3}\right) \delta=13.91\left(\mathrm{CH}_{3}\right), 22.33\left(\mathrm{CH}_{2}\right), 33.12\left(\mathrm{CH}_{2}\right), 35.32\left(\mathrm{CH}_{2}\right)$, $123.88\left(\mathrm{~d}, J_{\mathrm{CP}}=10.8 \mathrm{~Hz}, \mathrm{CH}\right), 128.33(2 \mathrm{CH}), 128.61(\mathrm{CH}), 128.78(\mathrm{CH}), 128.87$ $(2 \mathrm{CH}), 128.95(2 \mathrm{CH}), 129.01$ (overlapped, $5 \mathrm{CH}), 129.84\left(\mathrm{~d}, J_{\mathrm{PC}}=9.9 \mathrm{~Hz}, \mathrm{C}\right), 130.21$ $\left(\mathrm{d}, J_{\mathrm{CP}}=98.9 \mathrm{~Hz}, \mathrm{C}\right), 130.94\left(\mathrm{~d}, J_{\mathrm{CP}}=10.6 \mathrm{~Hz}, 2 \mathrm{CH}\right), 132.00\left(\mathrm{~d}, J_{\mathrm{CP}}=105.08 \mathrm{~Hz}, \mathrm{C}\right)$, $132.12\left(\mathrm{~d}, J_{\mathrm{CP}}=2.8 \mathrm{~Hz}, \mathrm{CH}\right), 132.89\left(\mathrm{~d}, J_{\mathrm{CP}}=1.7 \mathrm{~Hz}, \mathrm{CH}\right), 134.08\left(\mathrm{~d}, J_{\mathrm{CP}}=95.2 \mathrm{~Hz}\right.$, C), $134.56\left(\mathrm{~d}, J_{\mathrm{CP}}=15.2 \mathrm{~Hz}, \mathrm{C}\right), 142.76(\mathrm{C}), 143.94\left(\mathrm{~d}, J_{\mathrm{CP}}=27.1 \mathrm{~Hz}, \mathrm{C}\right), 149.22(\mathrm{~d}$, $\left.J_{\mathrm{CP}}=21.6 \mathrm{~Hz}, \mathrm{C}\right) ;{ }^{31} \mathrm{P}\{1 \mathrm{H}\} \mathrm{NMR}\left(121 \mathrm{MHz}, \mathrm{CDCl}_{3}\right) \delta=39.2$; HRMS Calcd. for $\mathrm{C}_{30} \mathrm{H}_{27} \mathrm{OP}[\mathrm{M}+\mathrm{H}+]$ 435.1872, Found: 435.1883 .

3d: green solid (409 mg, $0.90 \mathrm{mmol}, 45 \%$ yield from 1d); mp:223-225 ${ }^{\circ} \mathrm{C} ;{ }^{1} \mathrm{H}$ NMR $\left(300 \mathrm{MHz}, \mathrm{CDCl}_{3}\right) \delta=7.21-7.29(\mathrm{~m}, 2 \mathrm{H}), 7.32-7.48(\mathrm{~m}, 18 \mathrm{H}), 7.71-7.76(\mathrm{~m}, 1 \mathrm{H})$, 7.82-7.88 (m, 2H); ${ }^{13} \mathrm{C}$ NMR $\left(75 \mathrm{MHz}, \mathrm{CDCl}_{3}\right) \delta=124.10\left(\mathrm{~d}, J_{\mathrm{PC}}=10.8 \mathrm{~Hz}, \mathrm{CH}\right)$, $126.84(2 \mathrm{CH}), 126.91(2 \mathrm{CH}), 127.44(\mathrm{CH}), 128.73(2 \mathrm{CH}), 128.82(\mathrm{CH}), 128.97(\mathrm{~d}$, $\left.J_{\mathrm{CP}}=11.7 \mathrm{~Hz}, 2 \mathrm{CH}\right), 129.05\left(\mathrm{~d}, J_{\mathrm{CP}}=9.3 \mathrm{~Hz}, \mathrm{CH}\right), 129.06(2 \mathrm{CH}), 129.16(2 \mathrm{CH})$, $129.18\left(\mathrm{~d}, J_{\mathrm{CP}}=10.2 \mathrm{~Hz}, \mathrm{CH}\right), 129.47\left(\mathrm{~d}, J_{\mathrm{CP}}=5.8 \mathrm{~Hz}, 2 \mathrm{CH}\right), 129.97\left(\mathrm{~d}, J_{\mathrm{CP}}=98.8 \mathrm{~Hz}\right.$, C), $130.99\left(\mathrm{~d}, J_{\mathrm{CP}}=10.5 \mathrm{~Hz}, 2 \mathrm{CH}\right), 131.73\left(\mathrm{~d}, J_{\mathrm{CP}}=9.98 \mathrm{~Hz}, \mathrm{C}\right), 132.08\left(\mathrm{~d}, J_{\mathrm{CP}}=\right.$ $105.0 \mathrm{~Hz}, \mathrm{C}), 132.28\left(\mathrm{~d}, J_{\mathrm{PC}}=2.7 \mathrm{~Hz}, \mathrm{CH}\right), 133.02\left(\mathrm{~d}, J_{\mathrm{CP}}=1.5 \mathrm{~Hz}, \mathrm{CH}\right), 133.75(\mathrm{~d}$, $\left.J_{\mathrm{CP}}=95.2 \mathrm{~Hz}, \mathrm{C}\right), 134.40\left(\mathrm{~d}, J_{\mathrm{CP}}=14.9 \mathrm{~Hz}, \mathrm{C}\right), 140.25(\mathrm{C}), 140.36(\mathrm{C}), 143.83\left(\mathrm{~d}, J_{\mathrm{CP}}\right.$ $=26.8 \mathrm{~Hz}, \mathrm{C}), 149.96\left(\mathrm{~d}, J_{\mathrm{CP}}=21.3 \mathrm{~Hz}, \mathrm{C}\right) ;{ }^{31} \mathrm{P}\{1 \mathrm{H}\} \mathrm{NMR}\left(121 \mathrm{MHz}, \mathrm{CDCl}_{3}\right) \delta=$ 39.2; HRMS Calcd. for $\mathrm{C}_{32} \mathrm{H}_{23} \mathrm{OP}\left[\mathrm{M}+\mathrm{H}^{+}\right]$455.1559, Found: 455.1562

3e: yellow solid (261 mg, $0.64 \mathrm{mmol}, 32 \%$ yield from 1e), ${ }^{1} \mathrm{H}$ NMR (300 MHz, $\left.\mathrm{CDCl}_{3}\right) \delta=3.67(\mathrm{~s}, 3 \mathrm{H}), 6.62\left(\mathrm{~d}, J_{\mathrm{HH}}=8.4 \mathrm{~Hz}, 2 \mathrm{H}\right), 7.15-7.18(\mathrm{~m}, 1 \mathrm{H}), 7.22\left(\mathrm{~d}, J_{\mathrm{HH}}=\right.$ $8.7 \mathrm{~Hz}, 2 \mathrm{H}), 7.34-7.46(\mathrm{~m}, 10 \mathrm{H}), 7.66-7.72(\mathrm{~m}, 1 \mathrm{H}), 7.75-7.82(\mathrm{~m}, 2 \mathrm{H}) ;{ }^{13} \mathrm{CNMR}$ $\left(75 \mathrm{MHz}, \mathrm{CDCl}_{3}\right) \delta=55.06\left(\mathrm{CH}_{3}\right), 113.79(2 \mathrm{CH}), 123.71\left(\mathrm{~d}, J_{\mathrm{CP}}=10.8 \mathrm{~Hz}, \mathrm{CH}\right)$, $125.08\left(\mathrm{~d}, J_{\mathrm{CP}}=10.1 \mathrm{~Hz}, \mathrm{C}\right), 128.59(\mathrm{CH}), 128.77\left(\mathrm{~d}, J_{\mathrm{CP}}=10.4 \mathrm{~Hz}, \mathrm{CH}\right), 128.86(\mathrm{~d}$, $\left.J_{\mathrm{CP}}=12.2 \mathrm{~Hz}, 2 \mathrm{CH}\right), 128.94\left(\mathrm{~d}, J_{\mathrm{CP}}=9.6 \mathrm{~Hz}, \mathrm{CH}\right), 129.05(2 \mathrm{CH}), 129.09(2 \mathrm{CH})$, $130.24\left(\mathrm{~d}, J_{\mathrm{CP}}=98.48 \mathrm{~Hz}, \mathrm{C}\right), 13042\left(\mathrm{~d}, J_{\mathrm{CP}}=6.0 \mathrm{~Hz}, 2 \mathrm{CH}\right), 130.92\left(\mathrm{~d}, J_{\mathrm{CP}}=10.5 \mathrm{~Hz}\right.$, $2 \mathrm{CH}), 131.83\left(\mathrm{~d}, J_{\mathrm{CP}}=105.1 \mathrm{~Hz}, \mathrm{C}\right), 132.11\left(\mathrm{~d}, J_{\mathrm{CP}}=2.8 \mathrm{~Hz}, \mathrm{CH}\right), 132.92\left(\mathrm{~d}, J_{\mathrm{CP}}=1.8\right.$ $\mathrm{Hz}, \mathrm{CH}), 133.61\left(\mathrm{~d}, J_{\mathrm{CP}}=95.5 \mathrm{~Hz}, \mathrm{C}\right), 134.65\left(\mathrm{~d}, J_{\mathrm{CP}}=15.2 \mathrm{~Hz}, \mathrm{C}\right), 144.05\left(\mathrm{~d}, J_{\mathrm{CP}}=\right.$ $27.1 \mathrm{~Hz}, \mathrm{C}), 148.24\left(\mathrm{~d}, J_{\mathrm{CP}}=21.7 \mathrm{~Hz}, \mathrm{C}\right), 159.16(\mathrm{C}) ;{ }^{31} \mathrm{P}\{1 \mathrm{H}\}$ NMR $(121 \mathrm{MHz}$, $\left.\mathrm{CDCl}_{3}\right) \delta=39.2$; HRMS Calcd. for $\mathrm{C}_{27} \mathrm{H}_{21} \mathrm{O}_{2} \mathrm{P}\left[\mathrm{M}+\mathrm{H}^{+}\right]$409.1352, Found: 409.1354. 
3f: dark yellow solid (76 mg, $0.14 \mathrm{mmol}, 7 \%$ with $\mathrm{Pd}(\mathrm{OAc})_{2}$ and $316 \mathrm{mg}, 0.58 \mathrm{mmol}$, $29 \%$ with $\mathrm{Pd}\left(\mathrm{PPh}_{3}\right)_{2} \mathrm{Cl}_{2}$ yields from 1f); ${ }^{1} \mathrm{H} \mathrm{NMR}\left(300 \mathrm{MHz}, \mathrm{CDCl}_{3}\right) \delta=6.74\left(\mathrm{~d}, J_{\mathrm{HH}}\right.$ $=8.7 \mathrm{~Hz}, 2 \mathrm{H}), 6.98-7.14(\mathrm{~m}, 9 \mathrm{H}), 7.18-7.24(\mathrm{~m}, 4 \mathrm{H}), 7.32-7.55(\mathrm{~m}, 10 \mathrm{H}), 7.65-7.70$ $(\mathrm{m}, 1 \mathrm{H}), 7.83-7.88(\mathrm{~m}, 2 \mathrm{H}) ;{ }^{13} \mathrm{C} \mathrm{NMR}\left(75 \mathrm{MHz}, \mathrm{CDCl}_{3}\right) \delta=121.56(2 \mathrm{CH}), 123.44$ $(2 \mathrm{CH}), 123.63\left(\mathrm{~d}, J_{\mathrm{CP}}=10.8 \mathrm{~Hz}, \mathrm{CH}\right), 125.09(4 \mathrm{CH}), 125.65\left(\mathrm{~d}, J_{\mathrm{CP}}=10.3 \mathrm{~Hz}, \mathrm{C}\right)$, $128.58(\mathrm{CH}), 128.68\left(\mathrm{~d}, J_{\mathrm{CP}}=7.0 \mathrm{~Hz}, \mathrm{CH}\right), 128.79(\mathrm{CH}), 128.85(\mathrm{CH}), 128.94(2 \mathrm{CH})$, 129.01(CH), 129.14(2CH), $129.25(4 \mathrm{CH}), 129.91\left(\mathrm{~d}, J_{\mathrm{CP}}=6.4 \mathrm{~Hz}, 2 \mathrm{CH}\right), 130.60(\mathrm{~d}$, $\left.J_{\mathrm{CP}}=98.2 \mathrm{~Hz}, \mathrm{C}\right), 130.95\left(\mathrm{~d}, J_{\mathrm{CP}}=10.6 \mathrm{~Hz}, 2 \mathrm{CH}\right), 131.77\left(\mathrm{~d}, J_{\mathrm{CP}}=105.6 \mathrm{~Hz}, \mathrm{C}\right)$, $132.10\left(\mathrm{~d}, J_{\mathrm{CP}}=2.8 \mathrm{~Hz}, \mathrm{CH}\right), 132.92(\mathrm{CH}), 133.43\left(\mathrm{~d}, J_{\mathrm{CP}}=95.2 \mathrm{~Hz}, \mathrm{C}\right), 134.99(\mathrm{~d}$, $\left.J_{\mathrm{CP}}=15.6 \mathrm{~Hz}, \mathrm{C}\right), 144.25\left(\mathrm{~d}, J_{\mathrm{CP}}=26.9 \mathrm{~Hz}, \mathrm{C}\right), 147.05(\mathrm{C}), 147.37(\mathrm{C}), 147.87\left(\mathrm{~d}, J_{\mathrm{CP}}\right.$ $=21.8 \mathrm{~Hz}, \mathrm{C}) ;{ }^{31} \mathrm{P}\{1 \mathrm{H}\} \mathrm{NMR}\left(121 \mathrm{MHz}, \mathrm{CDCl}_{3}\right) \delta=39.1$.

3g: white solid (570 mg, $1.44 \mathrm{mmol}, 72 \%$ yield from 1g); mp:97-99 ${ }^{\circ} \mathrm{C}$; ${ }^{1} \mathrm{H}$ NMR $\left(300 \mathrm{MHz}, \mathrm{CDCl}_{3}\right) \delta=6.78(\mathrm{t}, J=8.4 \mathrm{~Hz}, 2 \mathrm{H}), 7.19-7.24(\mathrm{~m}, 3 \mathrm{H}), 7.30-7.48(\mathrm{~m}$, $10 \mathrm{H}), 7.68-7.79(\mathrm{~m}, 3 \mathrm{H}) ;{ }^{13} \mathrm{C} \mathrm{NMR}\left(75 \mathrm{MHz}, \mathrm{CDCl}_{3}\right) \delta=115.42(J=21.4 \mathrm{~Hz}, 2 \mathrm{CH})$, $124.11(\mathrm{~d}, J=10.8 \mathrm{~Hz}, \mathrm{CH}), 128.71\left(J_{1}=10.2 \mathrm{~Hz}, J_{2}=3.4 \mathrm{~Hz}, \mathrm{C}\right), 128.85(\mathrm{CH})$, $128.95(\mathrm{~d}, J=12.4 \mathrm{~Hz}, 2 \mathrm{CH}), 128.97(2 \mathrm{CH}), 129.10(2 \mathrm{CH}), 129.11(\mathrm{~d}, J=9.7 \mathrm{~Hz}$, $\mathrm{CH}), 129.23(\mathrm{~d}, J=10.6 \mathrm{~Hz}, \mathrm{CH}), 129.58\left(\mathrm{~d}, J_{\mathrm{CP}}=99.2 \mathrm{~Hz}, \mathrm{C}\right), 130.83(\mathrm{~d}, J=13.6$ $\mathrm{Hz}, \mathrm{CH}), 130.84(\mathrm{CH}), 130.91(\mathrm{~d}, J=10.4 \mathrm{~Hz}, 2 \mathrm{CH}), 131.53\left(\mathrm{~d}, J_{\mathrm{CP}}=105.7 \mathrm{~Hz}, \mathrm{C}\right)$, $132.33\left(\mathrm{~d}, J_{\mathrm{CP}}=2.7 \mathrm{~Hz}, \mathrm{CH}\right), 133.04\left(\mathrm{~d}, J_{\mathrm{CP}}=1.8 \mathrm{~Hz}, \mathrm{CH}\right), 133.18\left(\mathrm{~d}, J_{\mathrm{CP}}=95.8 \mathrm{~Hz}\right.$, C), $133.95(\mathrm{~d}, J=14.8 \mathrm{~Hz}, \mathrm{C}), 143.63(\mathrm{~d}, J=26.8 \mathrm{~Hz}, \mathrm{C}), 150.00(\mathrm{~d}, J=21.3 \mathrm{~Hz}, \mathrm{C})$, $162.18\left(\mathrm{~d}, J_{\mathrm{CF}}=246.8 \mathrm{~Hz}, \mathrm{C}\right) ;{ }^{31} \mathrm{P}\{1 \mathrm{H}\} \mathrm{NMR}\left(121 \mathrm{MHz}, \mathrm{CDCl}_{3}\right) \delta=39.4$; HRMS Calcd. for $\mathrm{C}_{26} \mathrm{H}_{18} \mathrm{FOP}\left[\mathrm{M}+\mathrm{H}^{+}\right]$397.1152, Found: 397.1155.

3h: yellow solid (607 mg, 1.36 mmol, $68 \%$ yield from $\mathbf{1 h}$ ); mp:87-89 ${ }^{\circ} \mathrm{C} ;{ }^{1} \mathrm{H}$ NMR $\left(300 \mathrm{MHz}, \mathrm{CDCl}_{3}\right) \delta=7.10-7.31(\mathrm{~m}, 15 \mathrm{H}), 7.55-7.61(\mathrm{~m}, 1 \mathrm{H}), 7.66-7.73(\mathrm{~m}, 2 \mathrm{H}) ;{ }^{13} \mathrm{C}$ NMR $\left(75 \mathrm{MHz}, \mathrm{CDCl}_{3}\right) \delta=123.82\left(\mathrm{q}, J_{\mathrm{CF}}=270.5 \mathrm{~Hz}, \mathrm{C}\right), 124.43\left(\mathrm{~d}, J_{\mathrm{CP}}=10.8 \mathrm{~Hz}\right.$, $\mathrm{CH}), 125.09\left(\mathrm{q}, J_{\mathrm{CF}}=3.6 \mathrm{~Hz}, 2 \mathrm{CH}\right), 128.76(2 \mathrm{CH}), 128.90(\mathrm{CH}), 129.02\left(\mathrm{~d}, J_{\mathrm{CP}}=6.0\right.$ $\mathrm{Hz}, \mathrm{CH}), 129.07(2 \mathrm{CH}), 129.12(2 \mathrm{CH}), 129.17\left(\mathrm{~d}, J_{\mathrm{CP}}=5.6 \mathrm{~Hz}, 2 \mathrm{CH}\right), 129.12\left(\mathrm{~d}, J_{\mathrm{CP}}\right.$ $=99.9 \mathrm{~Hz}, \mathrm{C}), 129.62\left(\mathrm{~d}, J_{\mathrm{CP}}=10.7 \mathrm{~Hz}, \mathrm{CH}\right), 129.73\left(\mathrm{q}, J_{\mathrm{CF}}=31.7 \mathrm{~Hz}, \mathrm{C}\right), 130.78(\mathrm{~d}$, $\left.J_{\mathrm{CP}}=10.6 \mathrm{~Hz}, 2 \mathrm{CH}\right), 131.86\left(\mathrm{~d}, J_{\mathrm{CP}}=105.7 \mathrm{~Hz}, \mathrm{C}\right), 132.43\left(\mathrm{~d}, J_{\mathrm{CP}}=2.4 \mathrm{~Hz}, \mathrm{CH}\right)$, $132.86\left(\mathrm{~d}, J_{\mathrm{CP}}=95.2 \mathrm{~Hz}, \mathrm{C}\right), 133.12(\mathrm{CH}), 133.25\left(\mathrm{~d}, J_{\mathrm{CP}}=14.3 \mathrm{~Hz}, \mathrm{C}\right), 136.58\left(\mathrm{~d}, J_{\mathrm{CP}}\right.$ $=9.4 \mathrm{~Hz}, \mathrm{C}), 143.15\left(\mathrm{~d}, J_{\mathrm{CP}}=26.3 \mathrm{~Hz}, \mathrm{C}\right), 151.86\left(\mathrm{~d}, J_{\mathrm{CP}}=20.7 \mathrm{~Hz}, \mathrm{C}\right) ;{ }^{31} \mathrm{P}\{1 \mathrm{H}\}$ NMR (121 MHz, $\left.\mathrm{CDCl}_{3}\right) \delta=38.9$; HRMS Calcd. for $\mathrm{C}_{27} \mathrm{H}_{18} \mathrm{~F}_{3} \mathrm{OP}\left[\mathrm{M}+\mathrm{H}^{+}\right]$447.1120, Found: 447.1124; Anal. Calcd. for $\mathrm{C}_{27} \mathrm{H}_{18} \mathrm{~F}_{3} \mathrm{OP}$ : C, 72.65; H, 4.06. Found: C, 72.10; H, 4.09 .

\section{Synthesis of 5 a.}




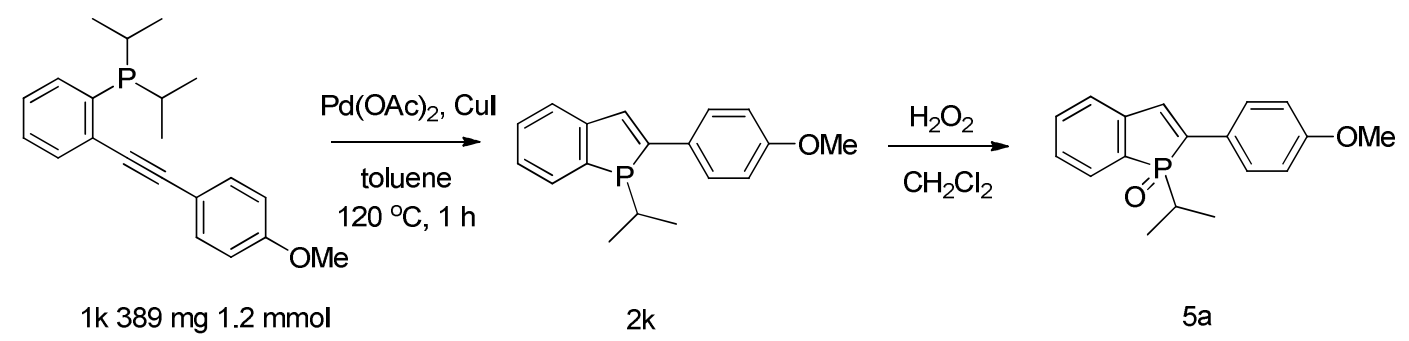

The procedure for the synthesis of benzophosphole $2 \mathbf{k}\left({ }^{31} \mathrm{P}\right.$ NMR: $\delta=13.1 \mathrm{ppm}$ in $\mathrm{CH}_{2} \mathrm{Cl}_{2}$ ) was according to 2, was chromatographed with Petroleum ether: $\mathrm{CH}_{2} \mathrm{Cl}_{2}$ (10:1), after oxided with $\mathrm{H}_{2} \mathrm{O}_{2}$, the mixture was filtered through a short pad of silica gel with EtOAc, the filtrate was evaporated, and the residue was chromatographed on silica gel with $\mathrm{CH}_{2} \mathrm{Cl}_{2}$ :EtOAc (1:1) as eluent to give pure benzophosphole oxide.

5a: light yellow oil (93 mg, $0.31 \mathrm{mmol}, 26 \%$ yield from 1k); ${ }^{1} \mathrm{H}$ NMR $(300 \mathrm{MHz}$, $\left.\mathrm{CDCl}_{3}\right) \delta=0.80-0.88(\mathrm{~m}, 3 \mathrm{H}), 1.22-1.30(\mathrm{~m}, 3 \mathrm{H}), 2.22-2.37(\mathrm{~m}, 1 \mathrm{H}), 3.81(\mathrm{~s}, 3 \mathrm{H})$, $6.91(\mathrm{~d}, 2 \mathrm{H}), 7.21-7.33(\mathrm{~m}, 3 \mathrm{H}), 7.41-7.47(\mathrm{~m}, 1 \mathrm{H}), 7.66-7.71(\mathrm{~m}, 1 \mathrm{H}), 7.75-7.78(\mathrm{~m}$, $2 \mathrm{H}) ;{ }^{13} \mathrm{C}$ NMR $\left(75 \mathrm{MHz}, \mathrm{CDCl}_{3}\right) \delta=15.15\left(\mathrm{~d}, J_{\mathrm{CP}}=2.5 \mathrm{~Hz}, \mathrm{CH}_{3}\right), 15.51\left(\mathrm{CH}_{3}\right), 28.07$ $\left(\mathrm{d}, J_{\mathrm{CP}}=66.4 \mathrm{~Hz}, \mathrm{CH}\right), 55.32\left(\mathrm{CH}_{3}\right), 114.38(2 \mathrm{CH}), 124.18\left(\mathrm{~d}, J_{\mathrm{CP}}=8.8 \mathrm{~Hz}, \mathrm{CH}\right)$, $126.32\left(\mathrm{~d}, J_{\mathrm{CP}}=10.4 \mathrm{~Hz}, \mathrm{C}\right), 128.00\left(\mathrm{~d}, J_{\mathrm{CP}}=8.6 \mathrm{~Hz}, 2 \mathrm{CH}\right), 128.04\left(\mathrm{~d}, J_{\mathrm{CP}}=2.7 \mathrm{~Hz}\right.$, $\mathrm{CH}), 129.00\left(\mathrm{~d}, J_{\mathrm{CP}}=9.4 \mathrm{~Hz}, \mathrm{CH}\right), 129.12\left(\mathrm{~d}, J_{\mathrm{CP}}=98.6 \mathrm{~Hz}, \mathrm{C}\right), 132.92(\mathrm{CH})$, $134.26\left(\mathrm{~d}, J_{\mathrm{CP}}=18.2 \mathrm{~Hz}, \mathrm{CH}\right), 136.34\left(\mathrm{~d}, J_{\mathrm{CP}}=85.3 \mathrm{~Hz}, \mathrm{C}\right), 142.29\left(\mathrm{~d}, J_{\mathrm{CP}}=25.6 \mathrm{~Hz}\right.$, C), $160.14(\mathrm{C}) ;{ }^{31} \mathrm{P}\{1 \mathrm{H}\}$ NMR $\left(121 \mathrm{MHz}, \mathrm{CDCl}_{3}\right) \delta=57.8$; HRMS Calcd. for $\mathrm{C}_{18} \mathrm{H}_{19} \mathrm{O}_{2} \mathrm{P}\left[\mathrm{M}+\mathrm{H}^{+}\right]$299.1195, Found: 299.1194

\section{Synthesis of $\mathbf{5 b}$.}

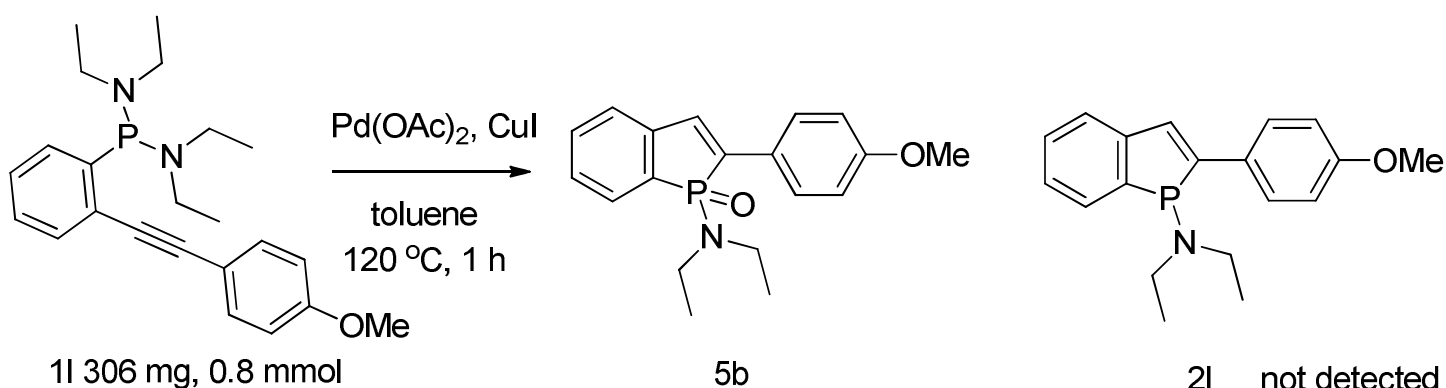

The procedure for the synthesis of benzophosphole $\mathbf{5 b}$ was according to $\mathbf{2}$, the mixture was filtered through a short pad of silica gel with EtOAc, the filtrate was evaporated, and the residue was chromatographed on silica gel with $\mathrm{CH}_{2} \mathrm{Cl}_{2}$ :EtOAc (5:1) as eluent to give pure benzophosphole oxide. In the reaction process, ${ }^{31} \mathrm{P}$ NMR of $\mathbf{2 l}$ was not observed, maybe it was oxided by the small amount of oxygen in the reaction system.

5b: light yellow solid (105 mg, $0.32 \mathrm{mmol}, 40 \%$ yield from 11); ${ }^{1} \mathrm{H}$ NMR (300 MHz, $\left.\mathrm{CDCl}_{3}\right) \delta=0.87\left(\mathrm{t}, J_{\mathrm{HH}}=8.1 \mathrm{~Hz}, 6 \mathrm{H}\right), 2.87-3.10(\mathrm{~m}, 4 \mathrm{H}), 3.72(\mathrm{~s}, 3 \mathrm{H}), 6.81-6.86(\mathrm{~m}$, 
2H), 7.08-7.32 (m, 4H), 7.43-7.62 (m, 1H), $7.64\left(\mathrm{~d}, J_{\mathrm{HH}}=8.4 \mathrm{~Hz}, 2 \mathrm{H}\right) ;{ }^{13} \mathrm{C} \mathrm{NMR}(75$

$\left.\mathrm{MHz}, \mathrm{CDCl}_{3}\right) \delta=14.15\left(\mathrm{~d}, J_{\mathrm{CP}}=2.0 \mathrm{~Hz}, \mathrm{CH}_{3}\right), 37.37\left(\mathrm{~d}, J_{\mathrm{CP}}=5.5 \mathrm{~Hz}, \mathrm{CH}_{2}\right), 55.20$

$\left(\mathrm{CH}_{3}\right), 114.17(2 \mathrm{CH}), 124.17\left(\mathrm{~d}, J_{\mathrm{CP}}=10.7 \mathrm{~Hz}, \mathrm{CH}\right), 125.64\left(\mathrm{~d}, J_{\mathrm{CP}}=10.0 \mathrm{~Hz}, \mathrm{C}\right)$,

$127.43\left(\mathrm{~d}, J_{\mathrm{CP}}=9.7 \mathrm{~Hz}, \mathrm{CH}\right), 127.88\left(\mathrm{~d}, J_{\mathrm{CP}}=6.5 \mathrm{~Hz}, 2 \mathrm{CH}\right), 128.28\left(\mathrm{~d}, J_{\mathrm{CP}}=10.6 \mathrm{~Hz}\right.$, $\mathrm{CH}), 130.64\left(\mathrm{~d}, J_{\mathrm{PC}}=124.7 \mathrm{~Hz}, \mathrm{C}\right), 132.62\left(\mathrm{~d}, J_{\mathrm{CP}}=1.9 \mathrm{~Hz}, \mathrm{CH}\right), 133.53\left(\mathrm{~d}, J_{\mathrm{CP}}=\right.$ $23.6 \mathrm{~Hz}, \mathrm{CH}), 136.13$ (d, $\left.J_{\mathrm{CP}}=111.9 \mathrm{~Hz}, \mathrm{C}\right), 140.89$ (d, $\left.J_{\mathrm{CP}}=32.3 \mathrm{~Hz}, \mathrm{C}\right), 159.91(\mathrm{C})$;

${ }^{31} \mathrm{P}\{1 \mathrm{H}\}$ NMR $\left(121 \mathrm{MHz}, \mathrm{CDCl}_{3}\right) \delta=43.7$; HRMS Calcd. for $\mathrm{C}_{19} \mathrm{H}_{22} \mathrm{NO}_{2} \mathrm{P}\left[\mathrm{M}+\mathrm{H}^{+}\right]$ 328.1461, Found: 328.1466 .

\section{Reference:}

1. Oskooie, H. A.; Heravi, M. M.; Behbahani, F. K. Molecules 2007, 12, 1438.

2. Chen, X.-K.; Zou, L.-Y.; Ren, A.-M.; Fan, J.-X. Phys. Chem.Chem. Phys. 2011, 13, 19490.

3. Xu, Y.-Z.; Wang, Z.-H.; Gan, Z.-J.; Xi, Q.-Z.; Duan, Z.; Mathey, F. Org. Lett. 2015, 17,1732 .

\section{X-ray Crystallographic Studies of Compound 4:}

- The single crystal of 4 suitable for X-ray analysis was grown in solution of dichloromethane, hexane. Data collections for $5 \mathrm{~b}$ were performed at $291.15 \mathrm{~K}$ on an oxford diffraction Gemini E diffractometer using graphite-monochromated Mo $\mathrm{Ka}$ radiation ( $\lambda=0.71073 \AA$ ). The structure of 4 was solved by use of SHELXTL program. Refinement was performed on F2 anisotropically for all the non-hydrogen atoms by the full-matrix least-squares method. Crystallographic data has been deposited with the Cambridge Crystallographic Data Center. CCDC reference number: 1057909 


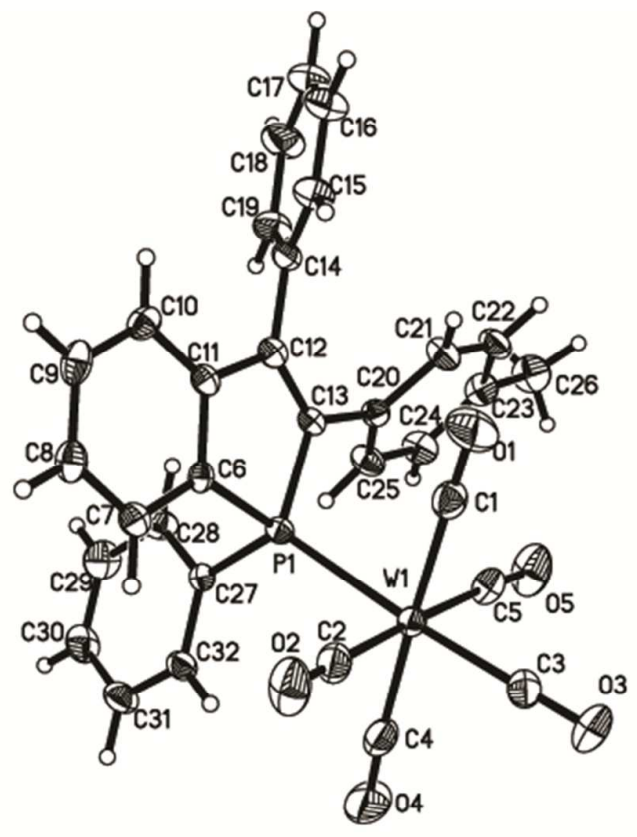

Table 1 Crystal data and structure refinement for 4 .

Identification code

4

Empirical formula

$\mathrm{C}_{32} \mathrm{H}_{21} \mathrm{O}_{5} \mathrm{PW}$

Formula weight

700.31

Temperature/K

291.15

Crystal system

monoclinic

Space group

$\mathrm{P} 2{ }_{1} / \mathrm{c}$

$\mathrm{a} / \AA$

$11.5210(4)$

$\mathrm{b} / \AA$

$11.9824(4)$

$\mathrm{c} / \AA$

$20.3830(6)$

$\alpha /{ }^{\circ}$

90

$\beta /{ }^{\circ}$

98.020(3)

$\gamma /{ }^{\circ}$

90

Volume $/ \AA^{3}$

2786.34(16)

Z

$\rho_{\text {calc }} \mathrm{g} / \mathrm{cm}^{3}$

4

$\mu / \mathrm{mm}^{-1}$

1.669

$\mathrm{F}(000)$

4.243

Crystal size $/ \mathrm{mm}^{3}$

1368.0

Radiation

$0.2 \times 0.17 \times 0.16$

$\operatorname{MoK} \alpha(\lambda=0.71073)$

$2 \Theta$ range for data collection $/{ }^{\circ} 6.682$ to 52.742 
Index ranges

Reflections collected $\quad 11762$

Independent reflections $\quad 5686\left[\mathrm{R}_{\text {int }}=0.0315, \mathrm{R}_{\text {sigma }}=0.0486\right]$

Data/restraints/parameters $\quad 5686 / 0 / 353$

Goodness-of-fit on $\mathrm{F}^{2} \quad 1.038$

Final $\mathrm{R}$ indexes $[\mathrm{I}>=2 \sigma(\mathrm{I})] \quad \mathrm{R}_{1}=0.0318, \mathrm{wR}_{2}=0.0562$

Final $\mathrm{R}$ indexes [all data] $\quad \mathrm{R}_{1}=0.0460, \mathrm{wR}_{2}=0.0610$

Largest diff. peak/hole / e $\AA^{-3} 0.52 /-0.91$

Table 1 Bond Lengths for 4.

\begin{tabular}{|c|c|c|c|c|c|}
\hline Atom & Atom & Length/Å & Atom & Atom & Length $/ \AA ̊$ \\
\hline $\mathrm{C} 1$ & $\mathrm{O} 1$ & $1.135(5)$ & C14 & C15 & $1.375(5)$ \\
\hline $\mathrm{C} 1$ & W1 & $2.038(5)$ & $\mathrm{C} 14$ & C19 & $1.381(5)$ \\
\hline $\mathrm{C} 2$ & $\mathrm{O} 2$ & $1.125(5)$ & $\mathrm{C} 15$ & $\mathrm{C} 16$ & $1.367(6)$ \\
\hline $\mathrm{C} 2$ & W1 & $2.046(5)$ & $\mathrm{C} 16$ & $\mathrm{C} 17$ & $1.383(7)$ \\
\hline C3 & $\mathrm{O} 3$ & $1.149(6)$ & $\mathrm{C} 17$ & C18 & $1.360(7)$ \\
\hline $\mathrm{C} 3$ & W1 & $1.988(5)$ & $\mathrm{C} 18$ & C19 & $1.385(6)$ \\
\hline $\mathrm{C} 4$ & $\mathrm{O} 4$ & $1.145(6)$ & $\mathrm{C} 20$ & $\mathrm{C} 21$ & $1.392(5)$ \\
\hline $\mathrm{C} 4$ & W1 & $2.021(5)$ & $\mathrm{C} 20$ & $\mathrm{C} 25$ & $1.391(5)$ \\
\hline $\mathrm{C} 5$ & $\mathrm{O} 5$ & $1.133(5)$ & $\mathrm{C} 21$ & $\mathrm{C} 22$ & $1.382(5)$ \\
\hline $\mathrm{C} 5$ & W1 & $2.041(5)$ & $\mathrm{C} 22$ & $\mathrm{C} 23$ & $1.383(5)$ \\
\hline C6 & $\mathrm{C} 7$ & $1.395(5)$ & $\mathrm{C} 23$ & $\mathrm{C} 24$ & $1.373(6)$ \\
\hline C6 & $\mathrm{C} 11$ & $1.403(5)$ & $\mathrm{C} 23$ & $\mathrm{C} 26$ & $1.514(5)$ \\
\hline C6 & $\mathrm{P} 1$ & $1.808(4)$ & $\mathrm{C} 24$ & $\mathrm{C} 25$ & $1.379(5)$ \\
\hline $\mathrm{C} 7$ & $\mathrm{C} 8$ & $1.389(6)$ & $\mathrm{C} 27$ & $\mathrm{C} 28$ & $1.383(5)$ \\
\hline $\mathrm{C} 8$ & C9 & $1.370(6)$ & $\mathrm{C} 27$ & $\mathrm{C} 32$ & $1.379(5)$ \\
\hline C9 & $\mathrm{C} 10$ & $1.391(5)$ & $\mathrm{C} 27$ & $\mathrm{P} 1$ & $1.834(3)$ \\
\hline $\mathrm{C} 10$ & C11 & $1.379(5)$ & $\mathrm{C} 28$ & $\mathrm{C} 29$ & $1.381(6)$ \\
\hline C11 & $\mathrm{C} 12$ & $1.490(5)$ & $\mathrm{C} 29$ & $\mathrm{C} 30$ & $1.368(6)$ \\
\hline $\mathrm{C} 12$ & $\mathrm{C} 13$ & $1.347(5)$ & C30 & C31 & $1.374(6)$ \\
\hline C12 & C14 & $1.483(5)$ & C31 & $\mathrm{C} 32$ & $1.383(5)$ \\
\hline C13 & $\mathrm{C} 20$ & $1.475(5)$ & $\mathrm{P} 1$ & W1 & $2.5173(11)$ \\
\hline C13 & $\mathrm{P} 1$ & $1.825(4)$ & & & \\
\hline
\end{tabular}

Table 2 Bond Angles for 4.

$\begin{array}{llllllll}\text { Atom } & \text { Atom } & \text { Atom } & \text { Angle }^{\circ} & \text { Atom } & \text { Atom } & \text { Atom } & \text { Angle }^{\circ} \\ \text { O1 } & \text { C1 } & \text { W1 } & 179.2(4) & \text { C22 } & \text { C23 } & \text { C26 } & 120.9(4) \\ \text { O2 } & \text { C2 } & \text { W1 } & 178.7(4) & \text { C24 } & \text { C23 } & \text { C22 } & 117.6(3) \\ \text { O3 } & \text { C3 } & \text { W1 } & 179.5(5) & \text { C24 } & \text { C23 } & \text { C26 } & 121.5(4) \\ \text { O4 } & \text { C4 } & \text { W1 } & 176.9(5) & \text { C23 } & \text { C24 } & \text { C25 } & 121.5(4)\end{array}$




$\begin{array}{llllllll}\mathrm{O} 5 & \mathrm{C} 5 & \mathrm{~W} 1 & 176.7(5) & \mathrm{C} 24 & \mathrm{C} 25 & \mathrm{C} 20 & 121.6(4) \\ \mathrm{C} 7 & \mathrm{C} 6 & \mathrm{C} 11 & 120.4(4) & \mathrm{C} 28 & \mathrm{C} 27 & \mathrm{P} 1 & 121.2(3) \\ \mathrm{C} 7 & \mathrm{C} 6 & \mathrm{P} 1 & 128.9(3) & \mathrm{C} 32 & \mathrm{C} 27 & \mathrm{C} 28 & 119.1(3) \\ \mathrm{C} 11 & \mathrm{C} 6 & \mathrm{P} 1 & 110.6(3) & \mathrm{C} 32 & \mathrm{C} 27 & \mathrm{P} 1 & 119.4(3) \\ \mathrm{C} 8 & \mathrm{C} 7 & \mathrm{C} 6 & 118.9(4) & \mathrm{C} 29 & \mathrm{C} 28 & \mathrm{C} 27 & 119.9(4) \\ \mathrm{C} 9 & \mathrm{C} 8 & \mathrm{C} 7 & 120.4(4) & \mathrm{C} 30 & \mathrm{C} 29 & \mathrm{C} 28 & 120.9(4) \\ \mathrm{C} 8 & \mathrm{C} 9 & \mathrm{C} 10 & 121.1(4) & \mathrm{C} 29 & \mathrm{C} 30 & \mathrm{C} 31 & 119.4(4) \\ \mathrm{C} 11 & \mathrm{C} 10 & \mathrm{C} 9 & 119.4(4) & \mathrm{C} 30 & \mathrm{C} 31 & \mathrm{C} 32 & 120.3(4) \\ \mathrm{C} 6 & \mathrm{C} 11 & \mathrm{C} 12 & 112.5(4) & \mathrm{C} 27 & \mathrm{C} 32 & \mathrm{C} 31 & 120.4(4) \\ \mathrm{C} 10 & \mathrm{C} 11 & \mathrm{C} 6 & 119.7(3) & \mathrm{C} 6 & \mathrm{P} 1 & \mathrm{C} 13 & 90.96(18) \\ \mathrm{C} 10 & \mathrm{C} 11 & \mathrm{C} 12 & 127.7(4) & \mathrm{C} 6 & \mathrm{P} 1 & \mathrm{C} 27 & 100.88(17) \\ \mathrm{C} 13 & \mathrm{C} 12 & \mathrm{C} 11 & 114.6(3) & \mathrm{C} 6 & \mathrm{P} 1 & \mathrm{~W} 1 & 116.38(12) \\ \mathrm{C} 13 & \mathrm{C} 12 & \mathrm{C} 14 & 125.9(3) & \mathrm{C} 13 & \mathrm{P} 1 & \mathrm{C} 27 & 106.75(16) \\ \mathrm{C} 14 & \mathrm{C} 12 & \mathrm{C} 11 & 119.5(4) & \mathrm{C} 13 & \mathrm{P} 1 & \mathrm{~W} 1 & 114.06(13) \\ \mathrm{C} 12 & \mathrm{C} 13 & \mathrm{C} 20 & 126.2(3) & \mathrm{C} 27 & \mathrm{P} 1 & \mathrm{~W} 1 & 122.65(14) \\ \mathrm{C} 12 & \mathrm{C} 13 & \mathrm{P} 1 & 111.1(3) & \mathrm{C} 1 & \mathrm{~W} 1 & \mathrm{C} 2 & 89.80(18) \\ \mathrm{C} 20 & \mathrm{C} 13 & \mathrm{P} 1 & 122.6(3) & \mathrm{C} 1 & \mathrm{~W} 1 & \mathrm{C} 5 & 90.00(18) \\ \mathrm{C} 15 & \mathrm{C} 14 & \mathrm{C} 12 & 121.5(4) & \mathrm{C} 1 & \mathrm{~W} 1 & \mathrm{P} 1 & 84.92(14) \\ \mathrm{C} 15 & \mathrm{C} 14 & \mathrm{C} 19 & 117.8(4) & \mathrm{C} 2 & \mathrm{~W} 1 & \mathrm{P} 1 & 90.53(14) \\ \mathrm{C} 19 & \mathrm{C} 14 & \mathrm{C} 12 & 120.7(3) & \mathrm{C} 3 & \mathrm{~W} 1 & \mathrm{C} 1 & 91.17(19) \\ \mathrm{C} 16 & \mathrm{C} 15 & \mathrm{C} 14 & 122.0(4) & \mathrm{C} 3 & \mathrm{~W} 1 & \mathrm{C} 2 & 89.71(19) \\ \mathrm{C} 15 & \mathrm{C} 16 & \mathrm{C} 17 & 119.5(4) & \mathrm{C} 3 & \mathrm{~W} 1 & \mathrm{C} 4 & 87.2(2) \\ \mathrm{C} 18 & \mathrm{C} 17 & \mathrm{C} 16 & 119.6(5) & \mathrm{C} 3 & \mathrm{~W} 1 & \mathrm{C} 5 & 87.64(19) \\ \mathrm{C} 17 & \mathrm{C} 18 & \mathrm{C} 19 & 120.5(5) & \mathrm{C} 3 & \mathrm{~W} 1 & \mathrm{P} 1 & 176.08(14) \\ \mathrm{C} 14 & \mathrm{C} 19 & \mathrm{C} 18 & 120.6(4) & \mathrm{C} 4 & \mathrm{~W} 1 & \mathrm{C} 1 & 177.8(2) \\ \mathrm{C} 21 & \mathrm{C} 20 & \mathrm{C} 13 & 121.5(3) & \mathrm{C} 4 & \mathrm{~W} 1 & \mathrm{C} 2 & 91.7(2) \\ \mathrm{C} 25 & \mathrm{C} 20 & \mathrm{C} 13 & 122.0(3) & \mathrm{C} 4 & \mathrm{~W} 1 & \mathrm{C} 5 & 88.4(2) \\ \mathrm{C} 25 & \mathrm{C} 20 & \mathrm{C} 21 & 116.5(3) & \mathrm{C} 4 & \mathrm{~W} 1 & \mathrm{P} 1 & 96.74(15) \\ \mathrm{C} 22 & \mathrm{C} 21 & \mathrm{C} 20 & 121.5(4) & \mathrm{C} 5 & \mathrm{~W} 1 & \mathrm{C} 2 & 177.34(19) \\ \mathrm{C} 21 & \mathrm{C} 22 & \mathrm{C} 23 & 121.2(4) & \mathrm{C} 5 & \mathrm{~W} 1 & \mathrm{P} 1 & 92.10(14)\end{array}$




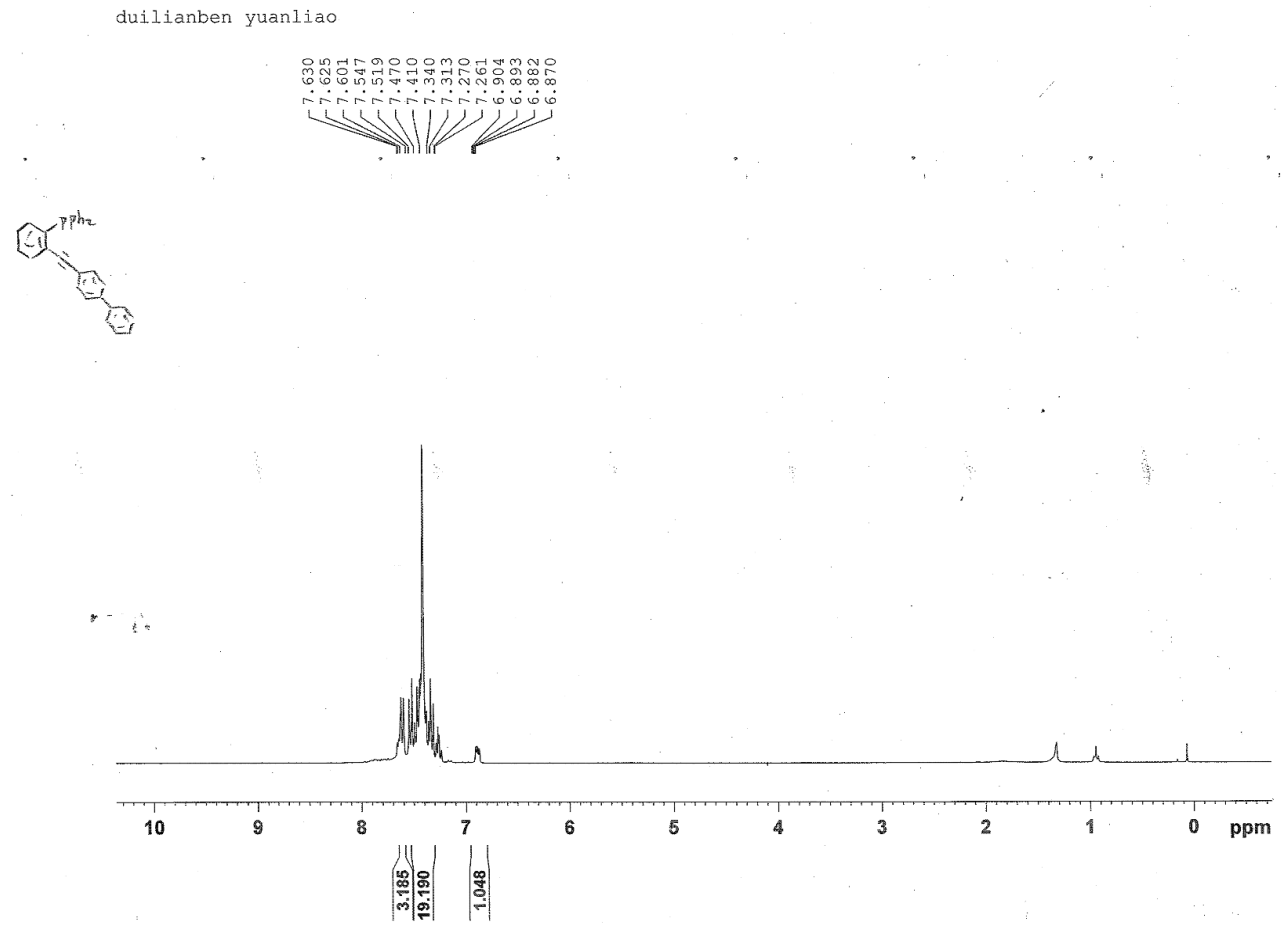

1d ${ }^{1} \mathrm{H}$ NMR

duilianben yuanliao

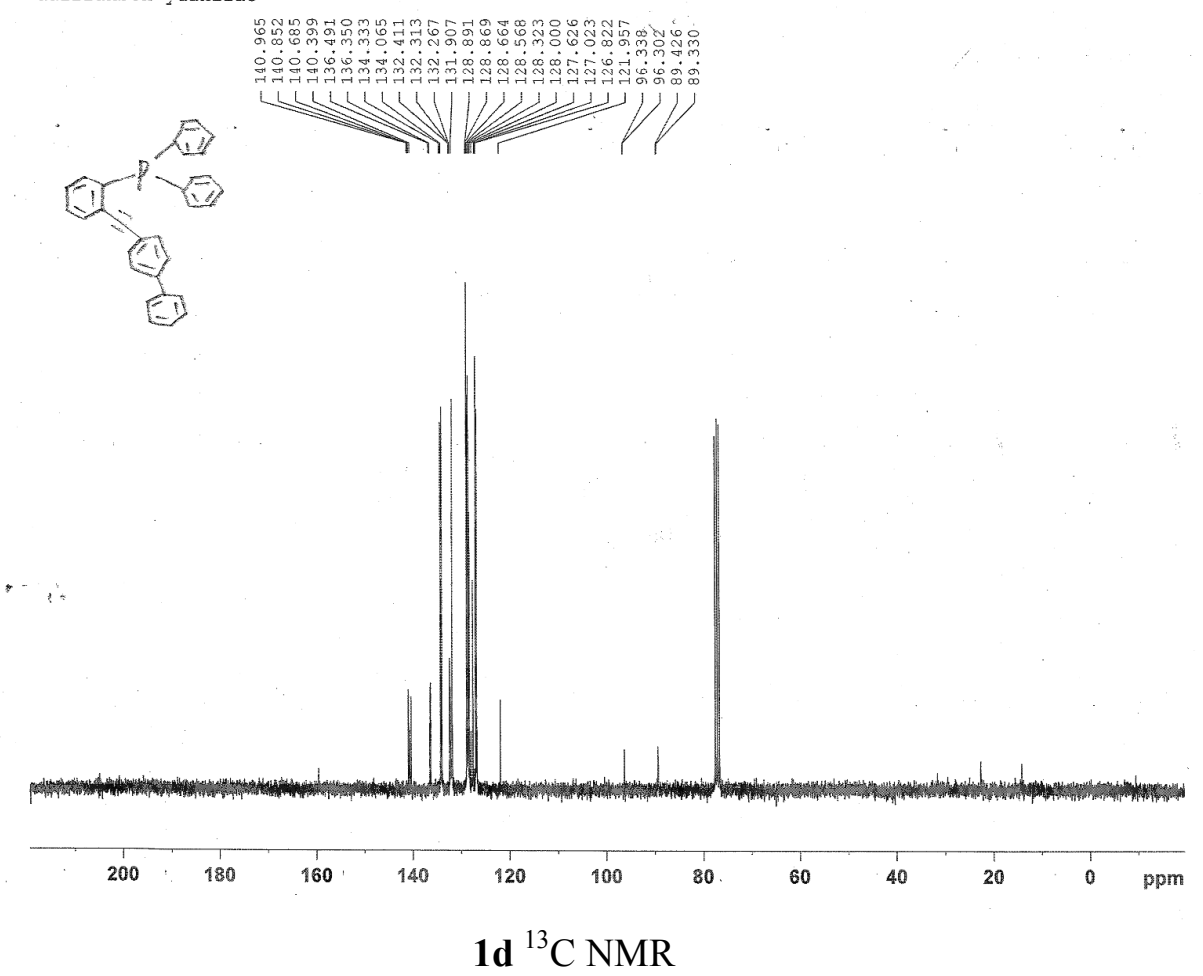

S-13 


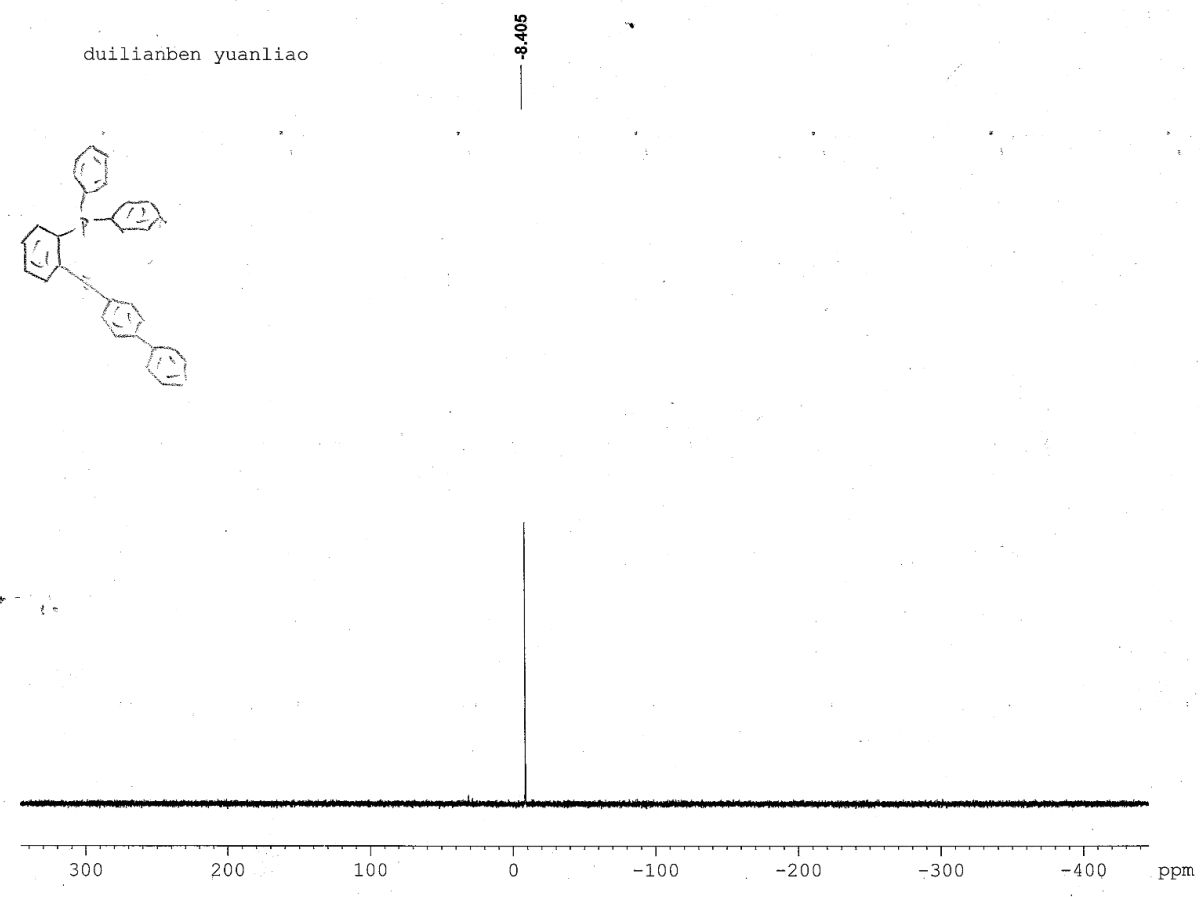

\section{1d ${ }^{31} \mathrm{P}$ NMR}

sanbenan yuaniiao

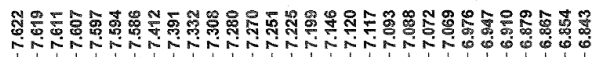

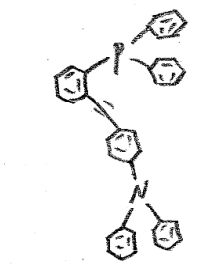

1f ${ }^{1} \mathrm{H}$ NMR

S-14 
sanbenan yuaniiao C1 $3 C P D$

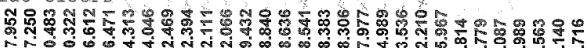

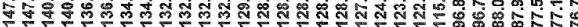

+1
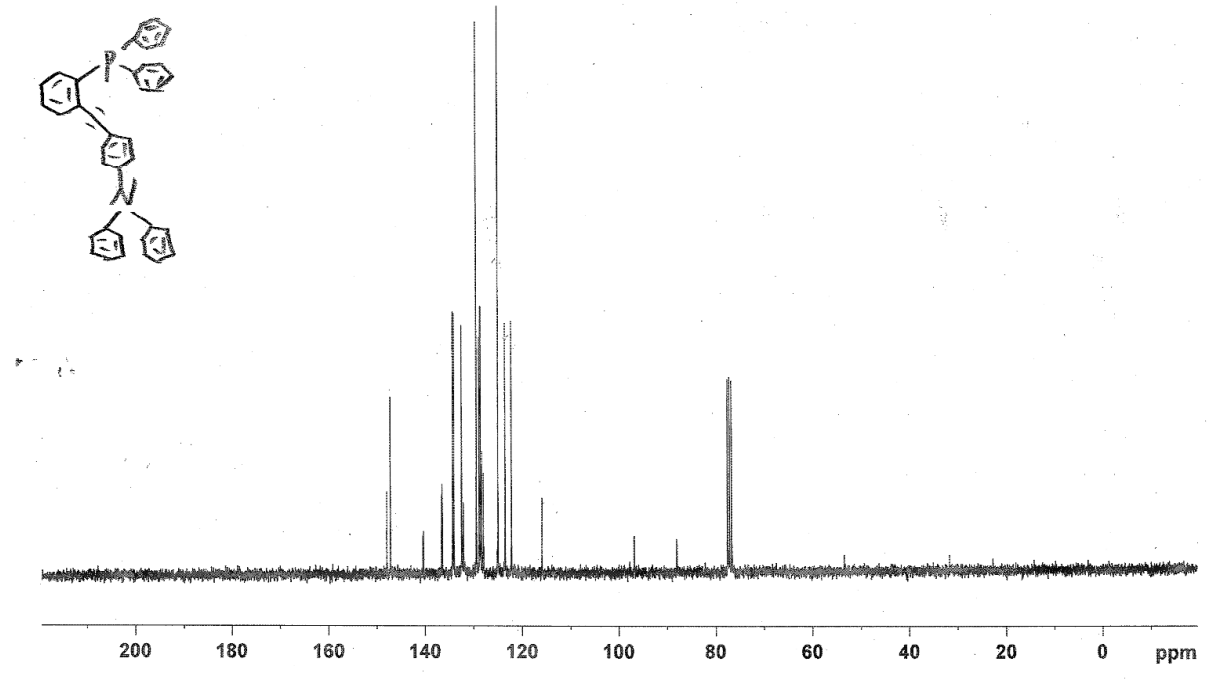

1f ${ }^{13} \mathrm{C}$ NMR

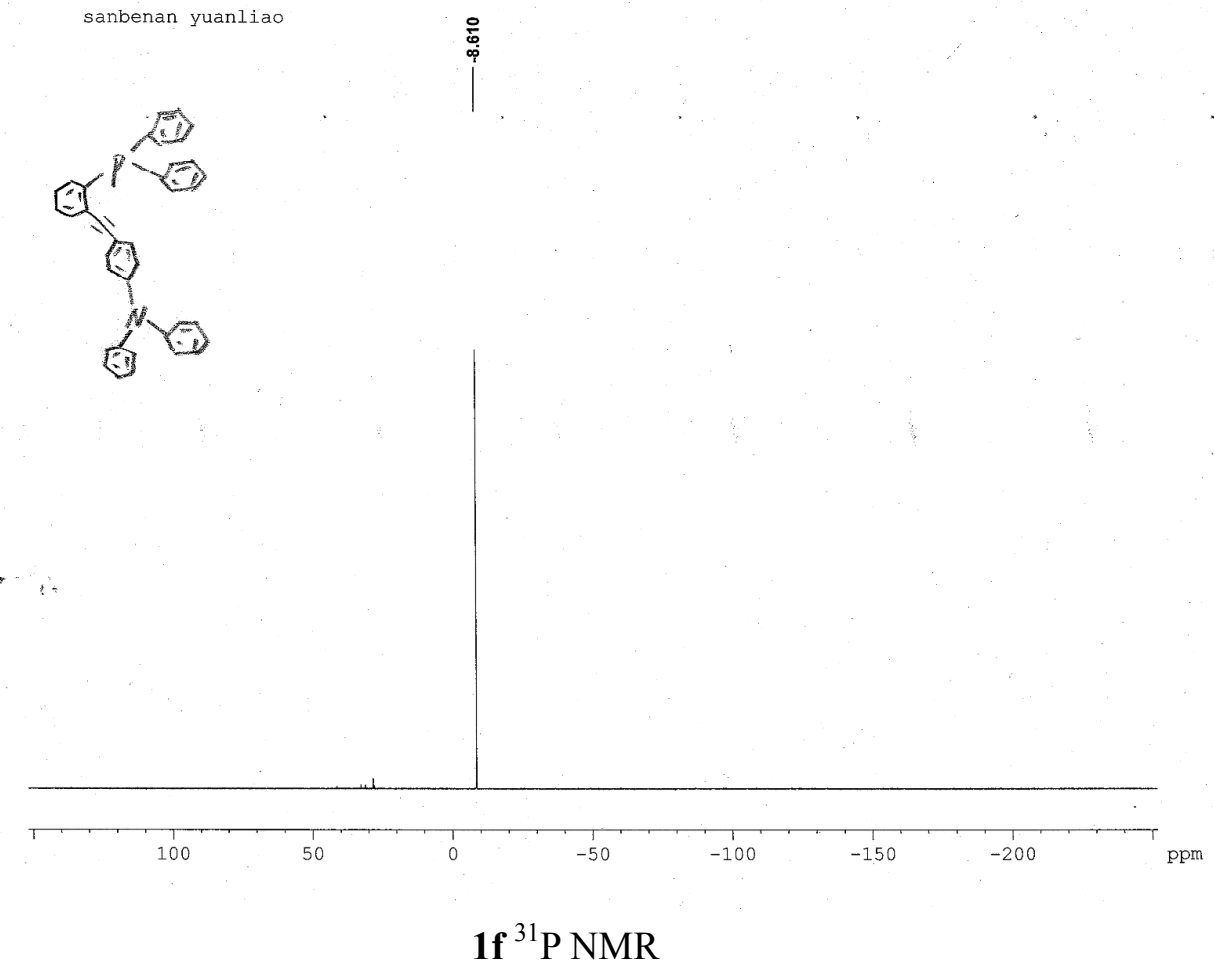

S-15 


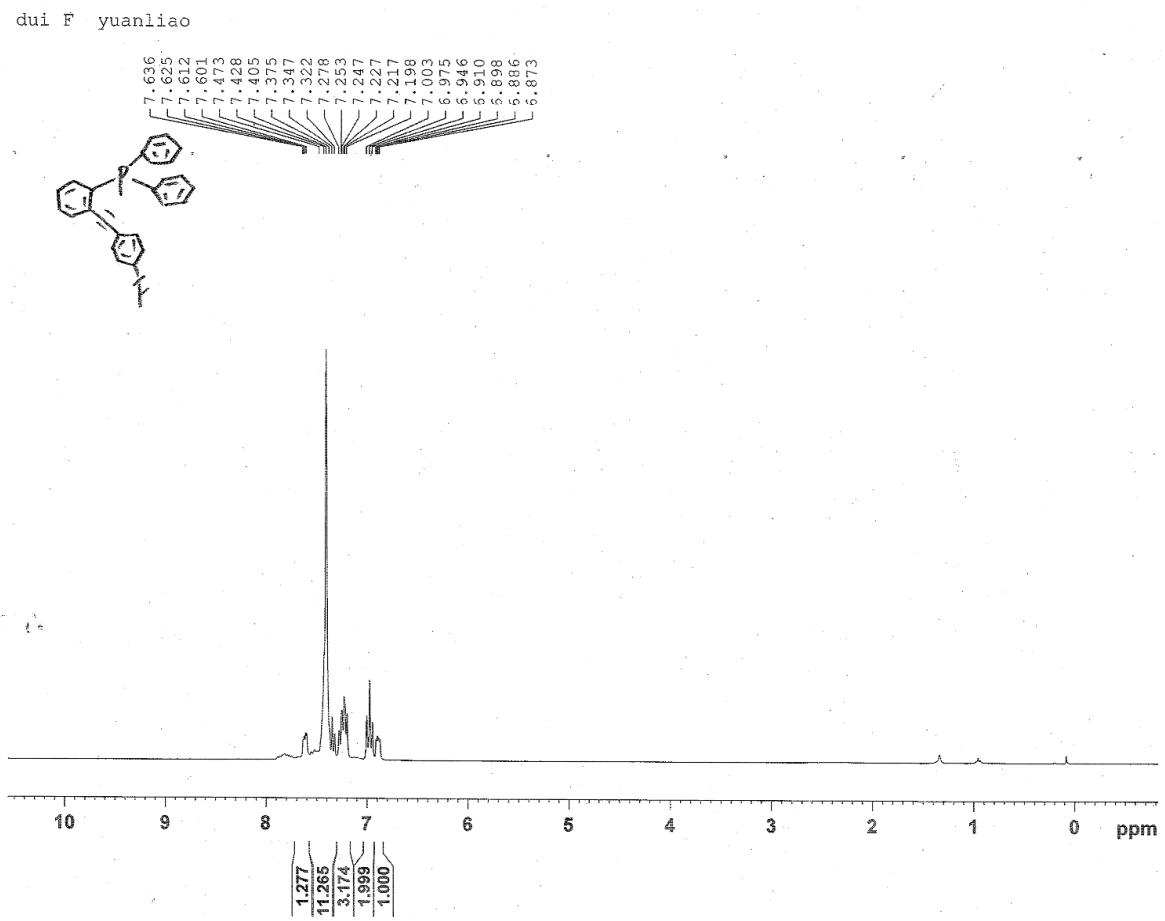

1g ${ }^{1} \mathrm{H}$ NMR
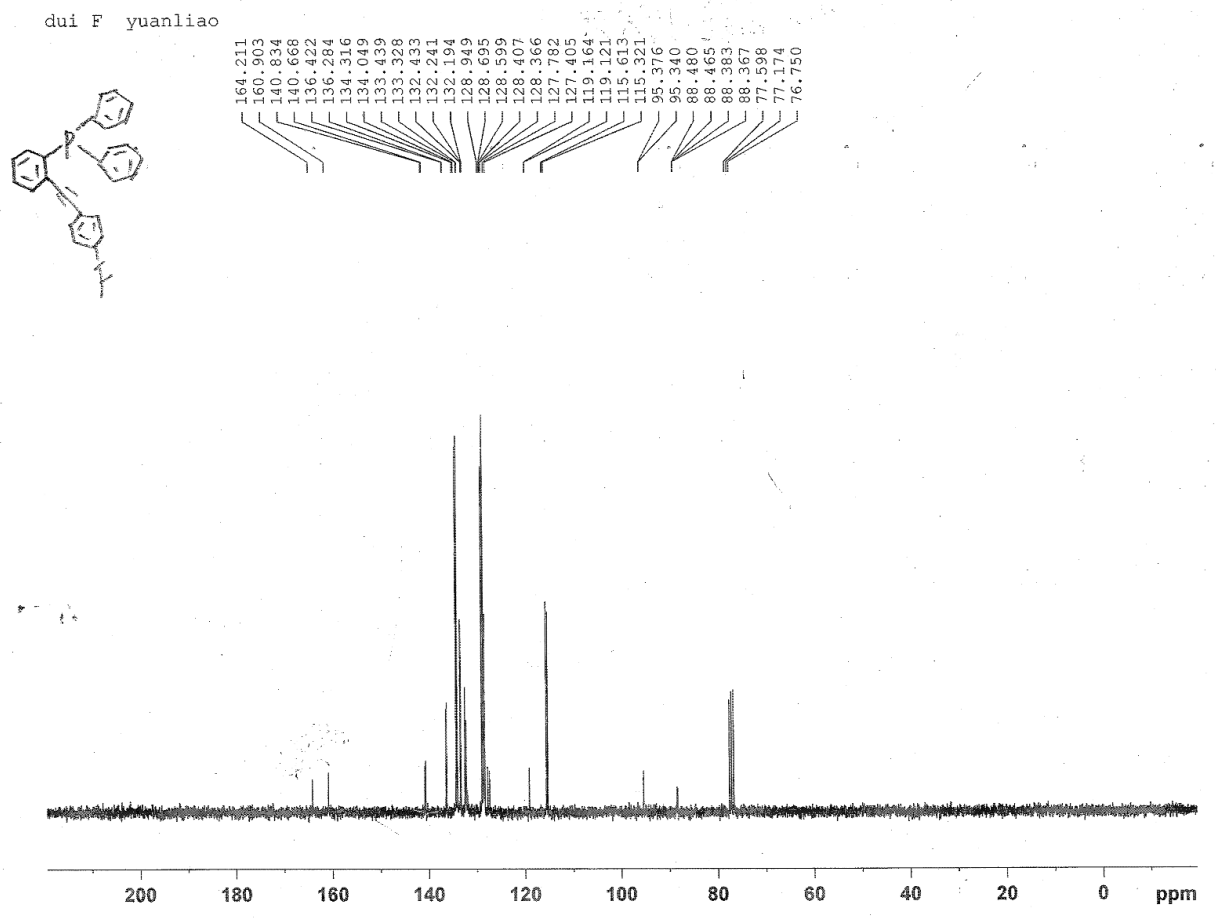

1g ${ }^{13} \mathrm{C}$ NMR 


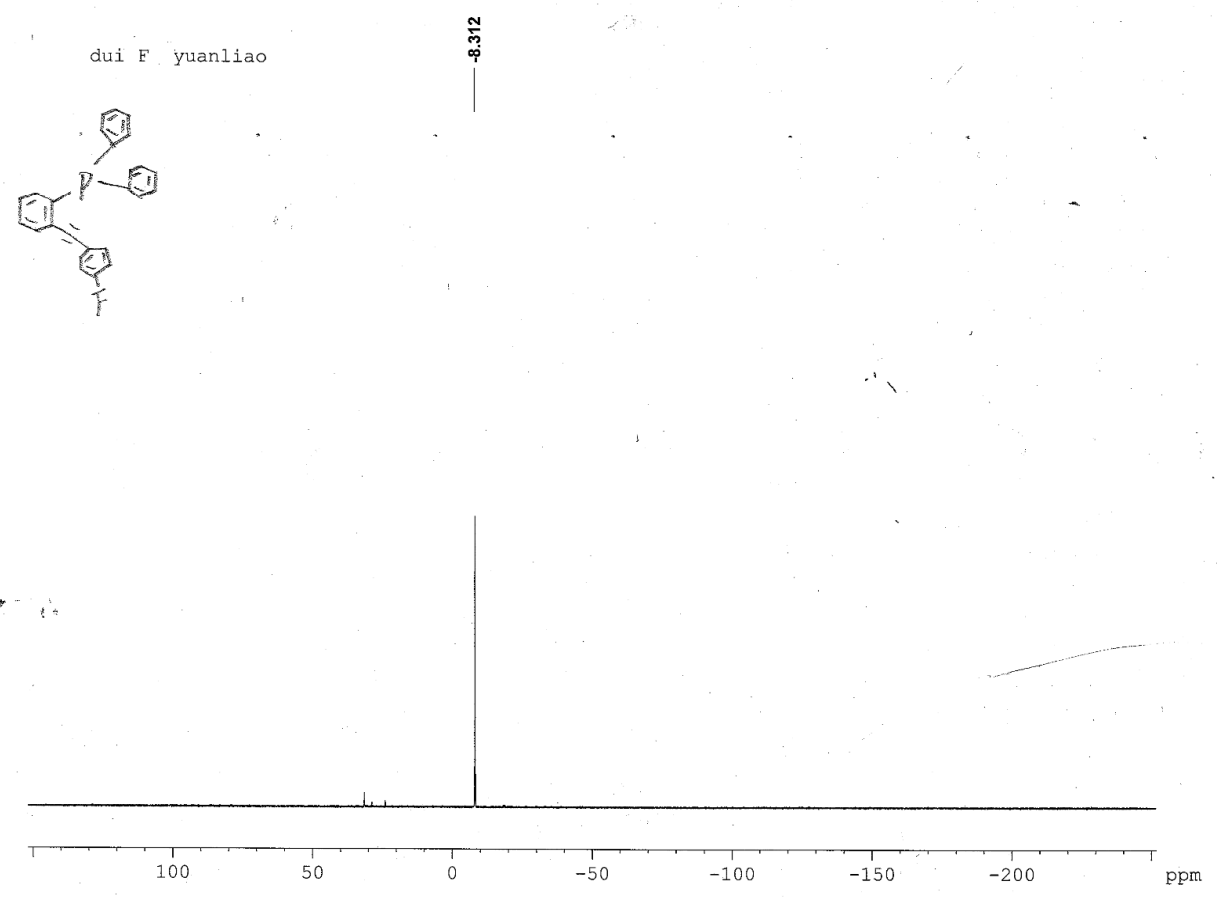

$1{ }^{31}$ P NMR

dui san F jiaji yuanliao

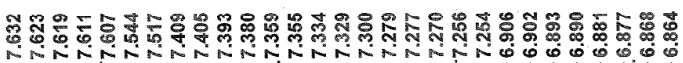

$a^{2}=2$

ats
$\therefore$

10
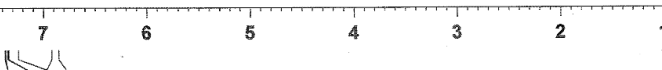

$0 \mathrm{ppm}$

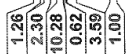

1h ${ }^{1} \mathrm{H}$ NMR 
subo C13CPD dui san F jiaji yuanliao
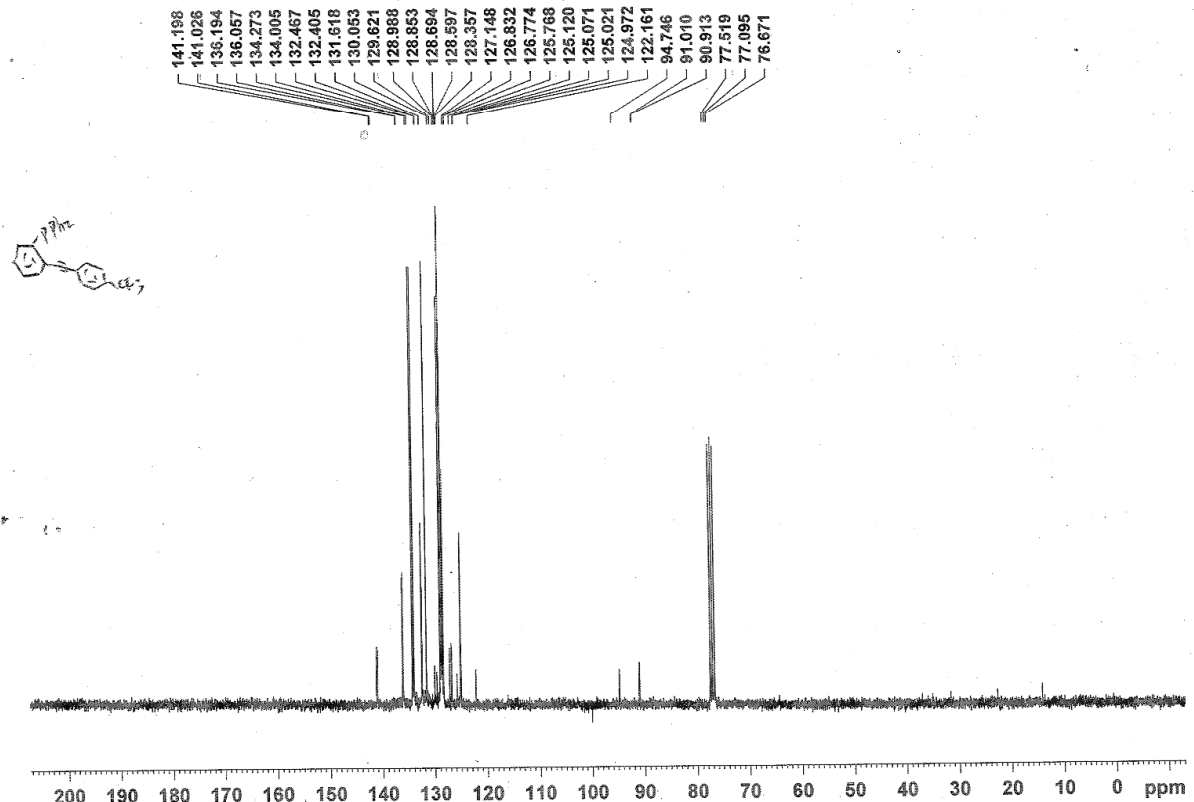

1h ${ }^{13} \mathrm{C}$ NMR

su bo P31 dui san F jiaji yuanliao

a

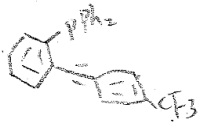

के

$\because$

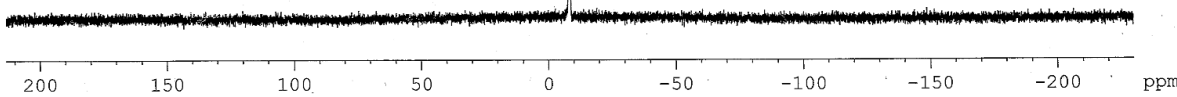

1h ${ }^{31}$ P NMR 
yibingjiyuanliaoyanghuawl

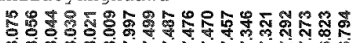

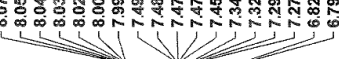

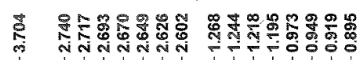<smiles></smiles>

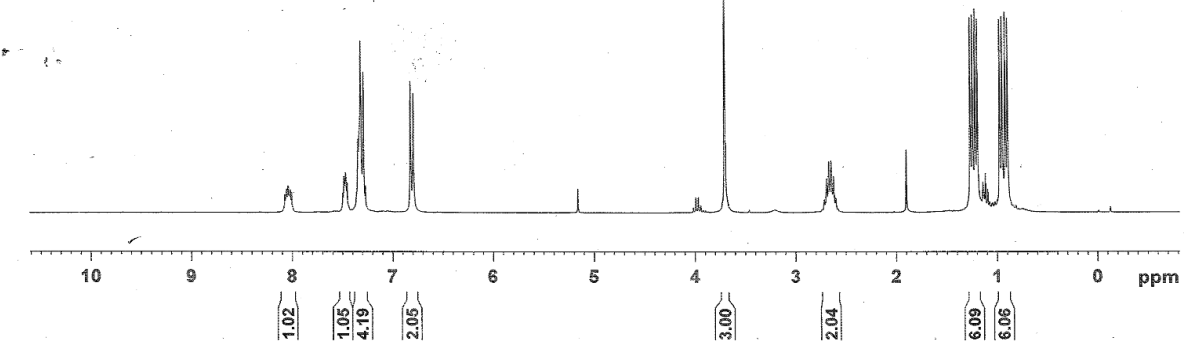

1k $P$-oxide ${ }^{1} \mathrm{H}$ NMR

yibingji yuanliaoyanghuawu
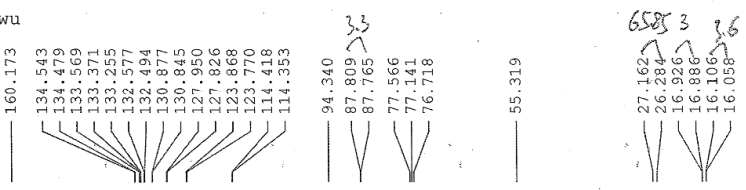

$\mathrm{C}^{2}$

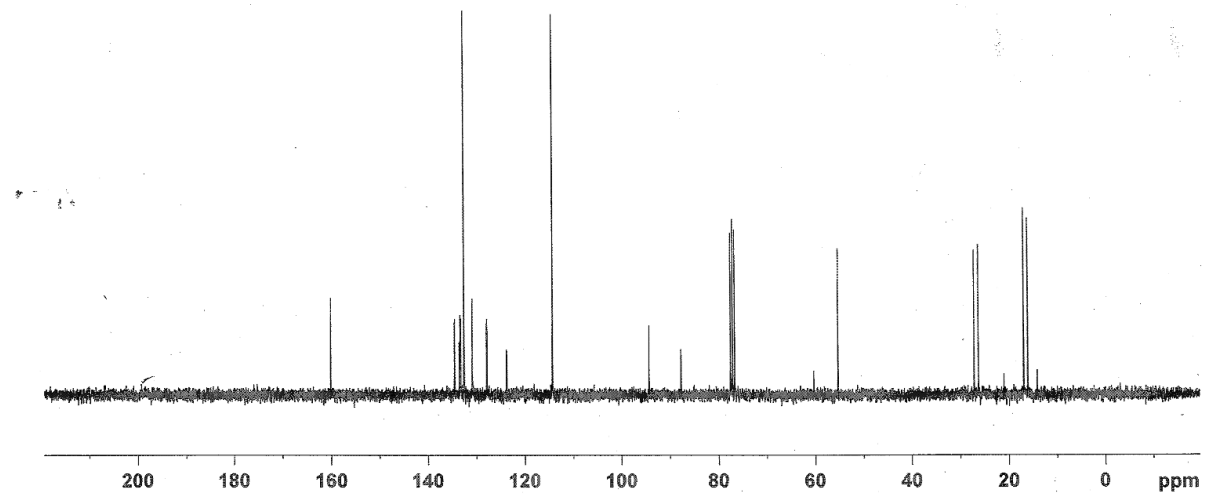

1k $P$-oxide ${ }^{13} \mathrm{C}$ NMR 
yibingjiyuanliaoyanghuawu
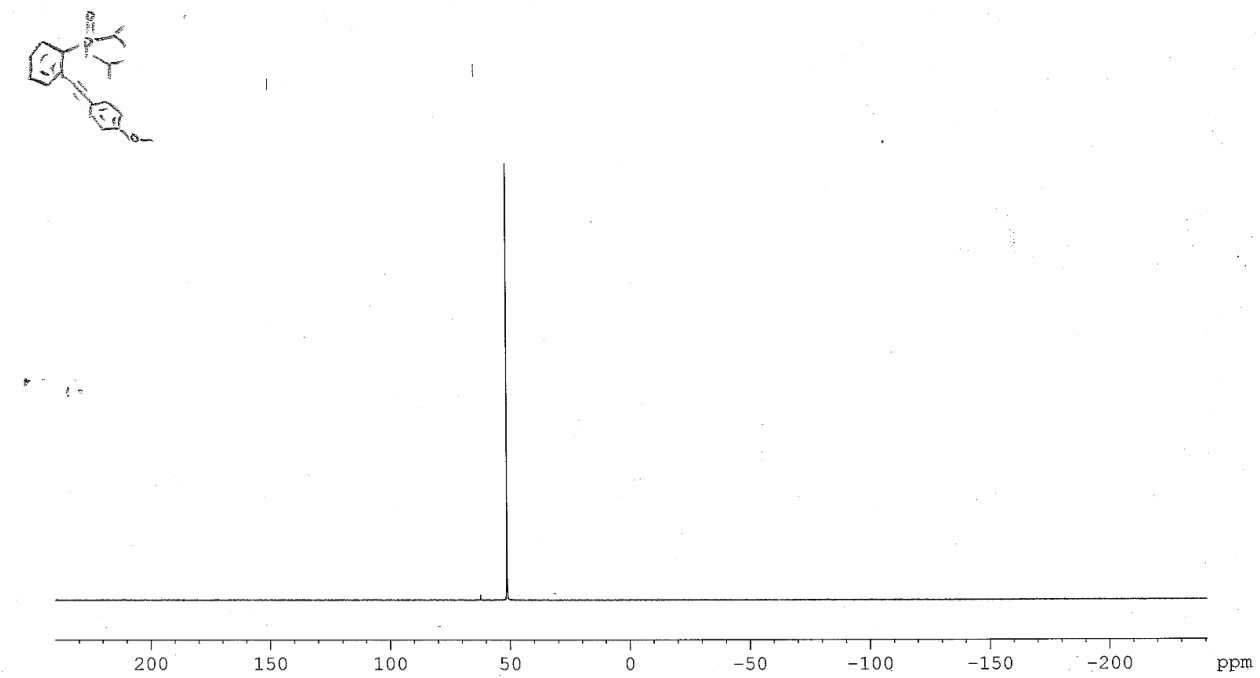

1k $P$-oxide ${ }^{31} \mathrm{P}$ NMR

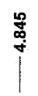
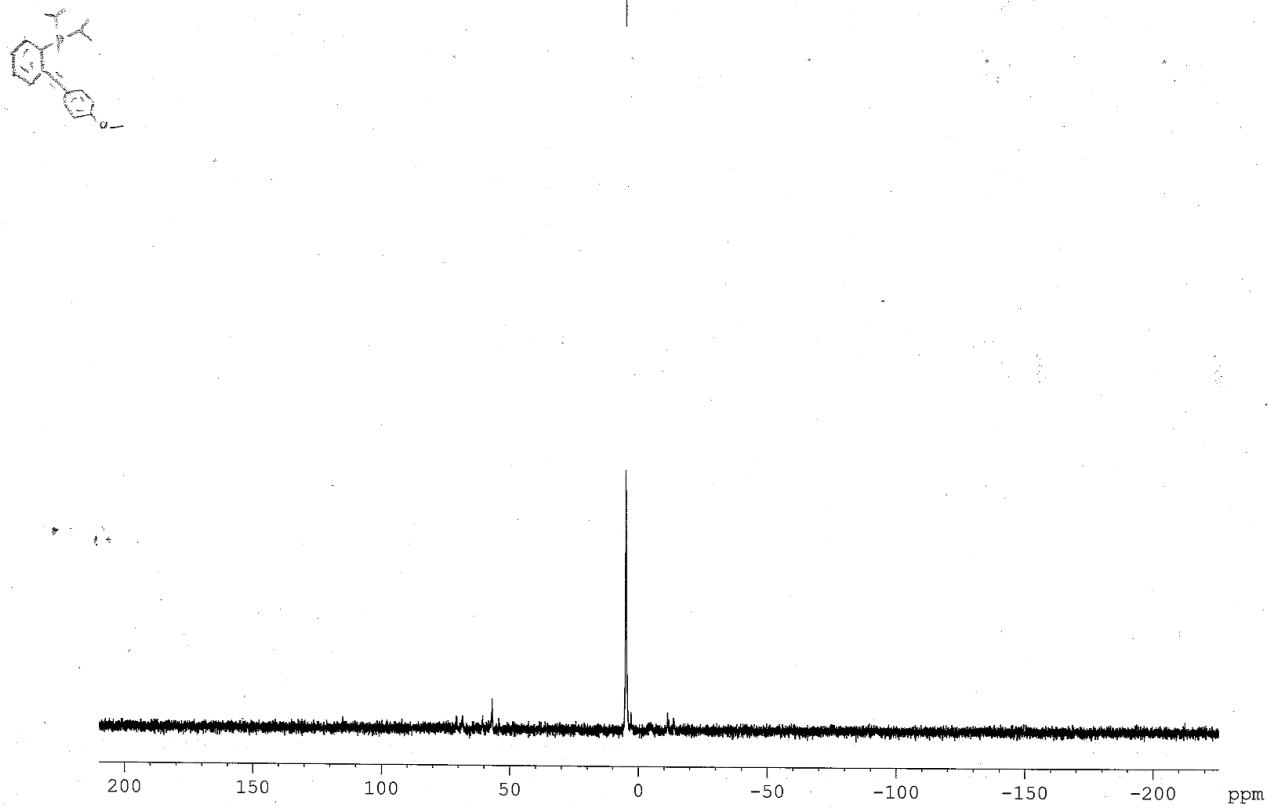

$\mathbf{1 k}{ }^{31} \mathrm{P}$ NMR 


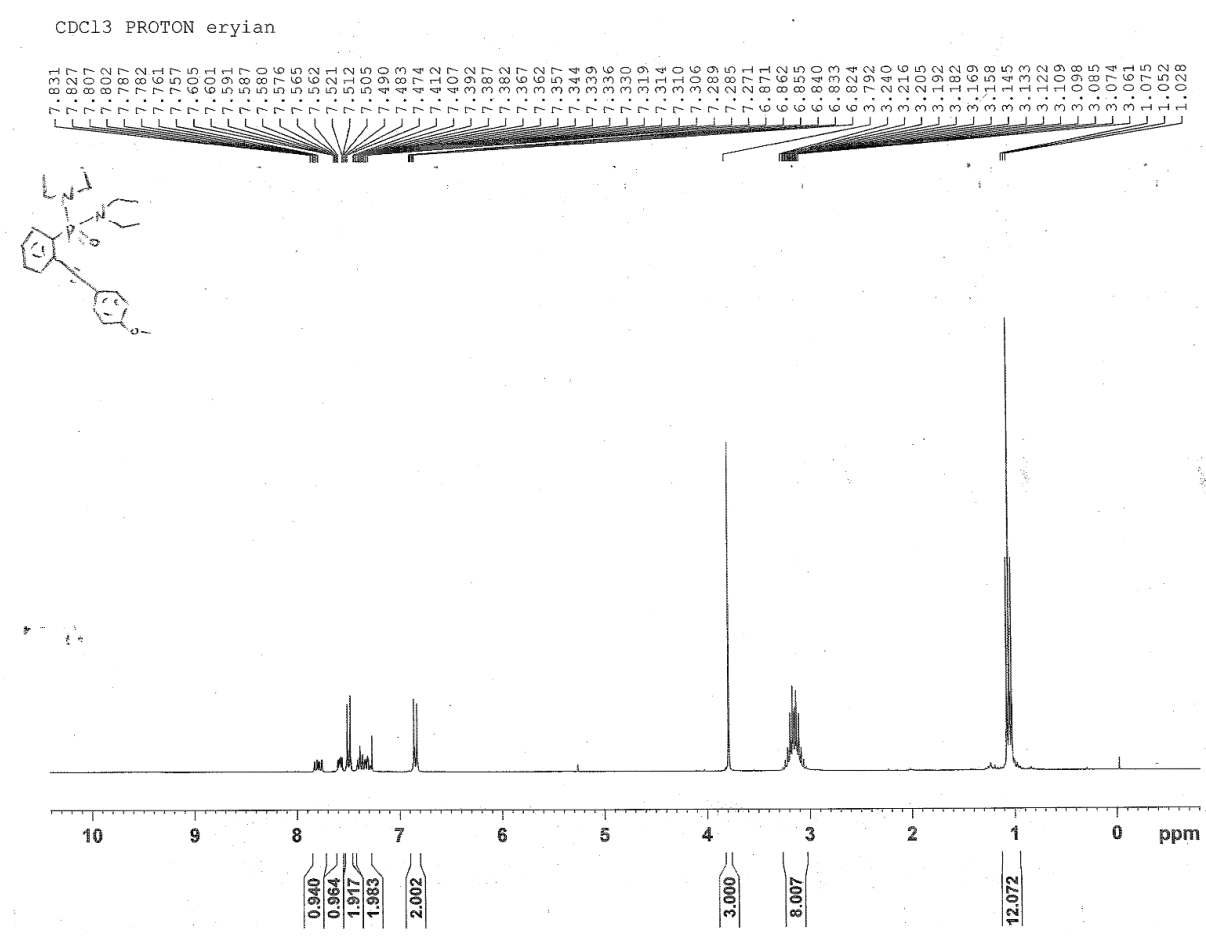

11 -oxide ${ }^{1} \mathrm{H}$ NMR
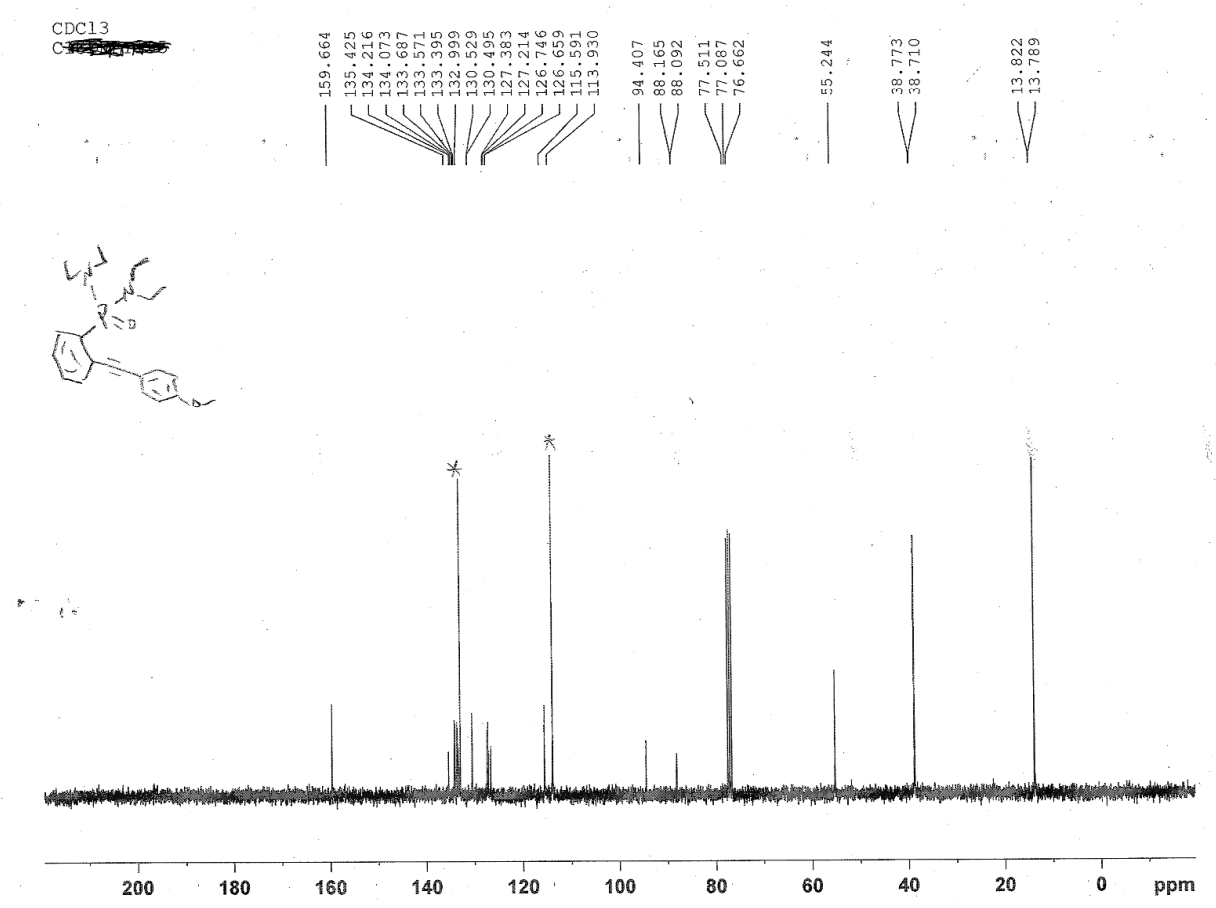

11 -oxide ${ }^{13} \mathrm{C}$ NMR 

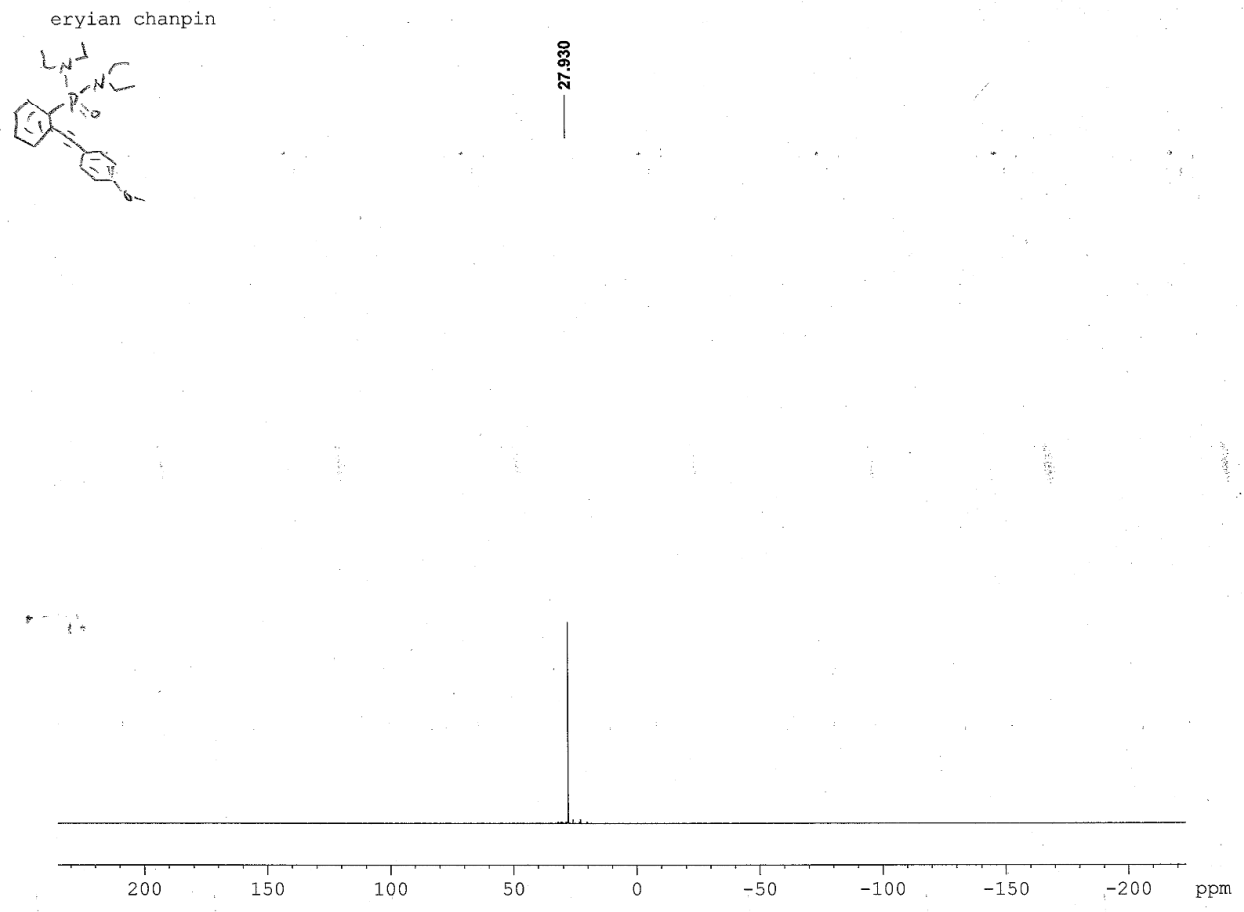

$11 P$-oxide ${ }^{31} \mathrm{P}$ NMR

|艹
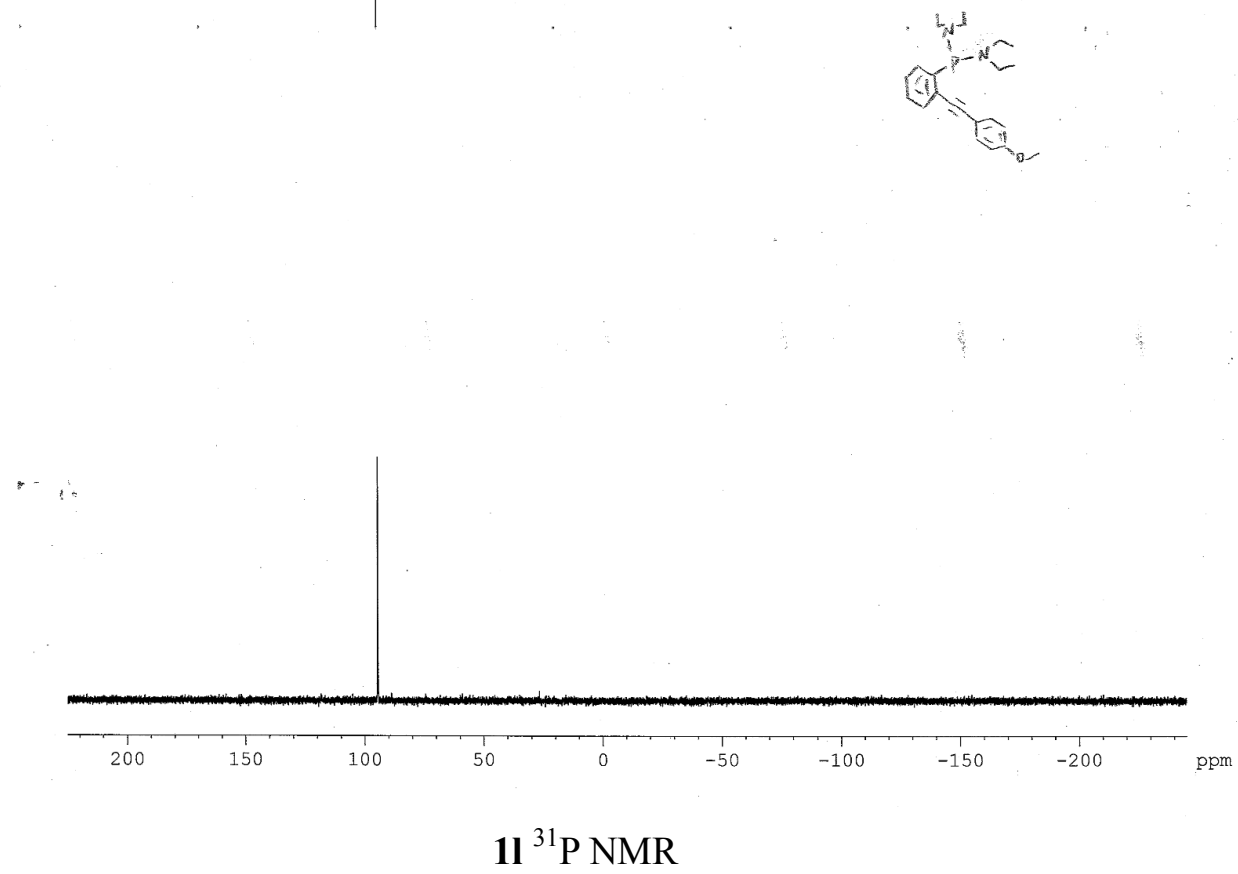

S-22 
cuihuajiajipeiwuwu

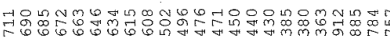

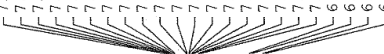

(witys

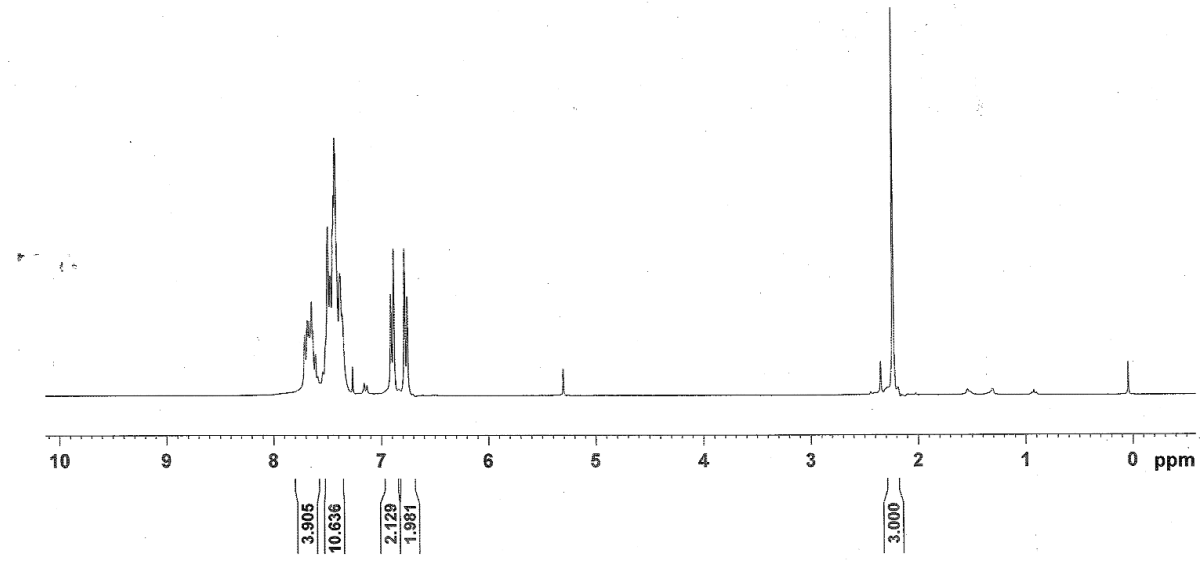

$4{ }^{1} \mathrm{H}$ NMR

cuihuajiajipeiwuwu C13CPD

\%

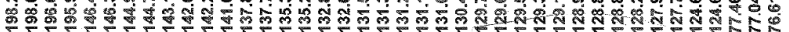
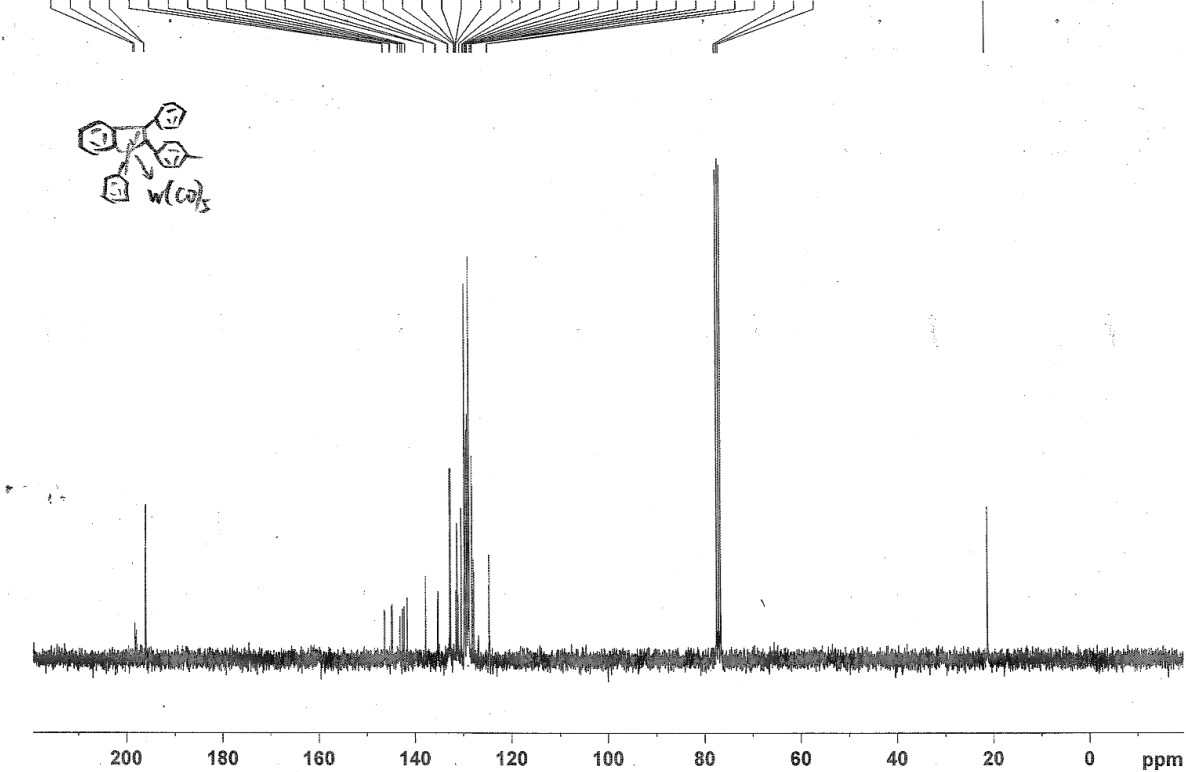

$4{ }^{13} \mathrm{C}$ NMR 


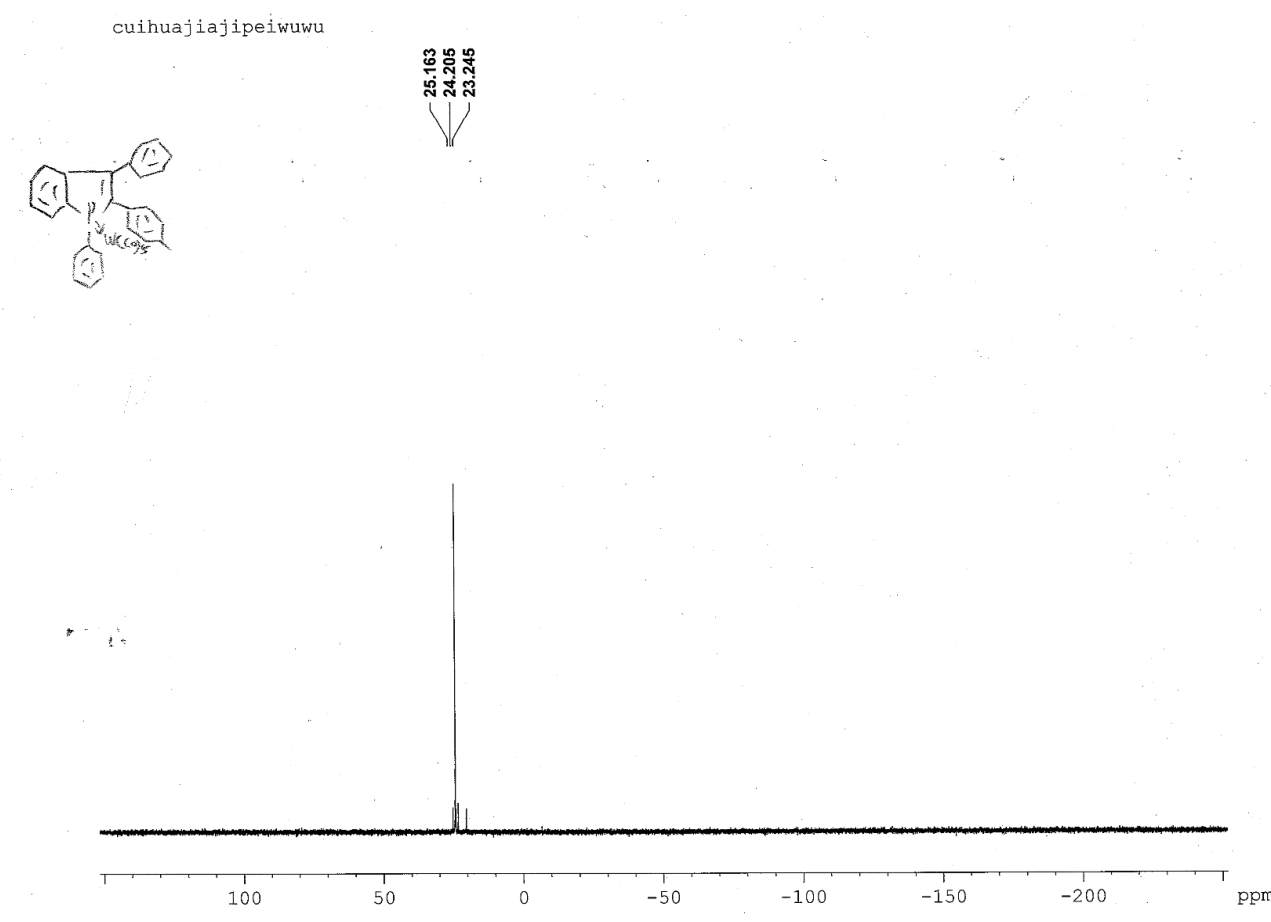

\section{$4^{31} \mathrm{P}$ NMR}
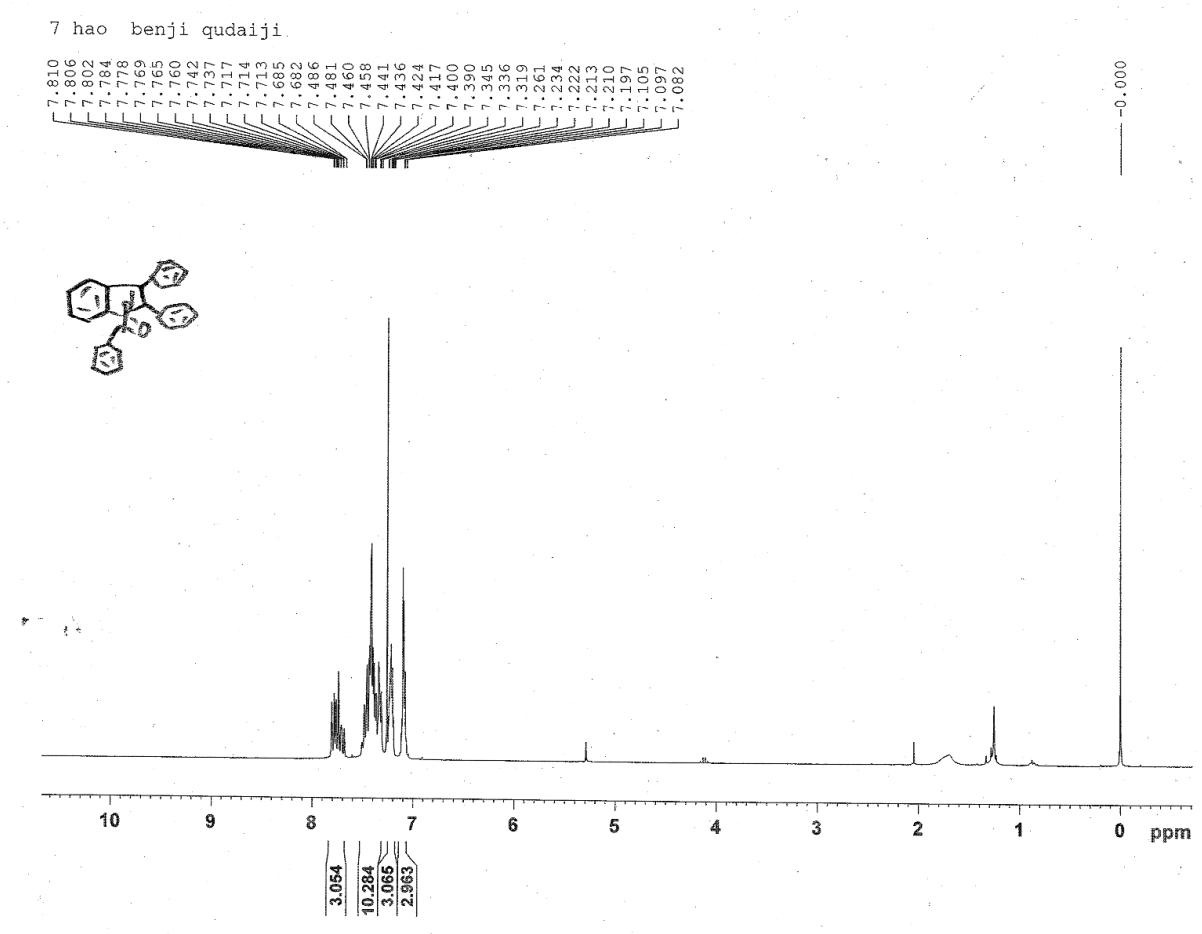

3a ${ }^{1} \mathrm{H}$ NMR 


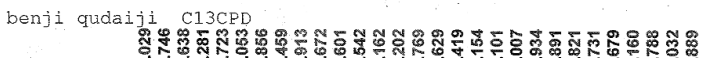

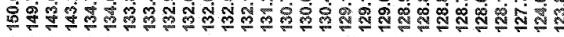

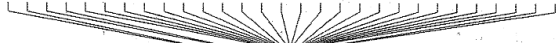

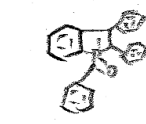

(1)

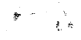

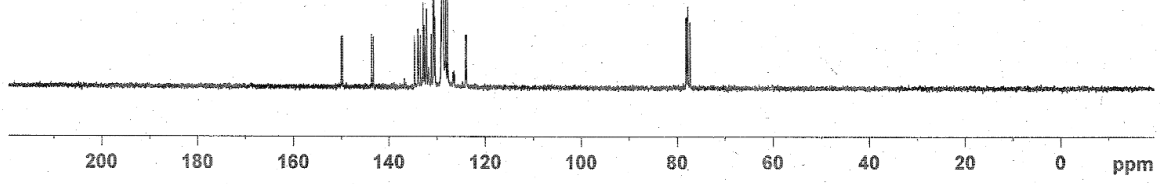

3a ${ }^{13}$ C NMR
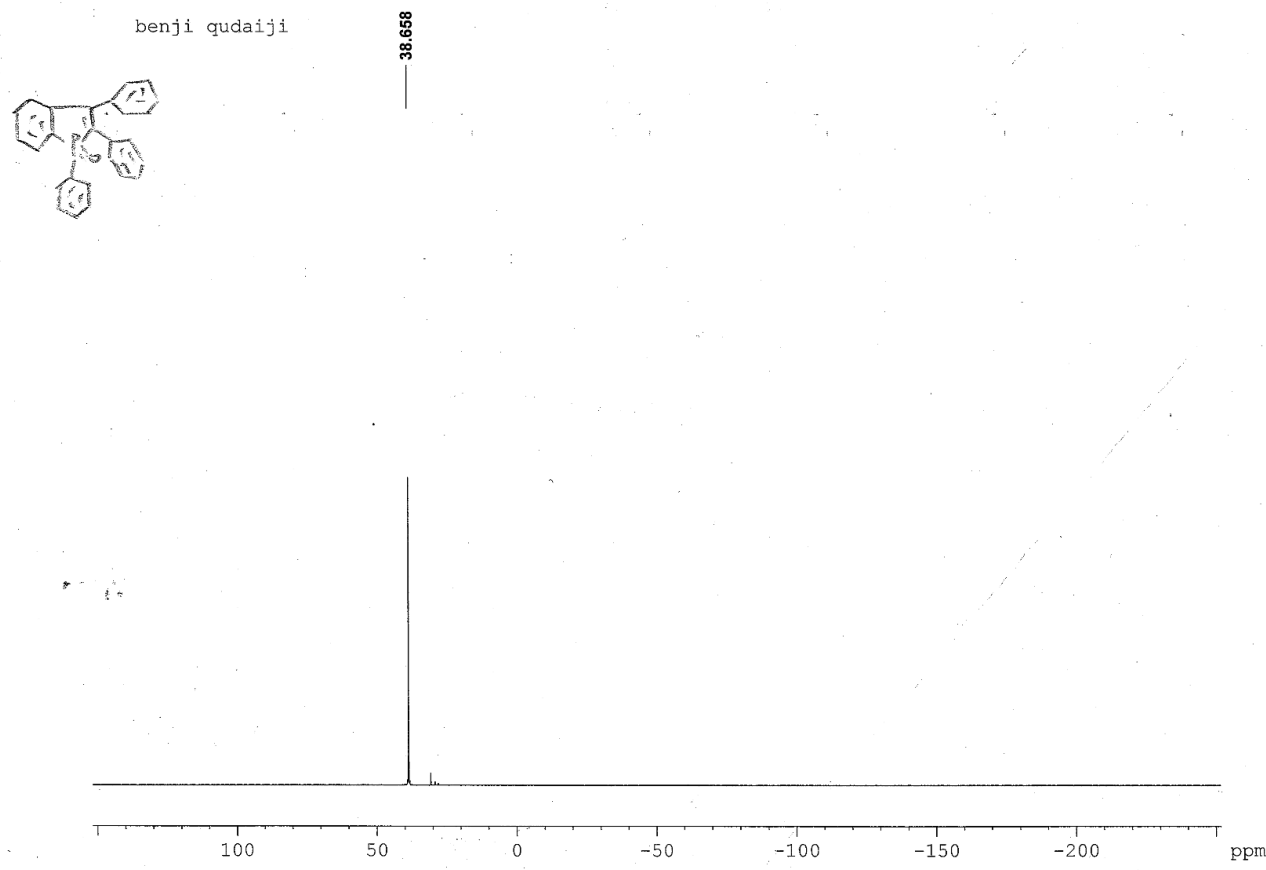

3a ${ }^{31}$ P NMR

S-25 


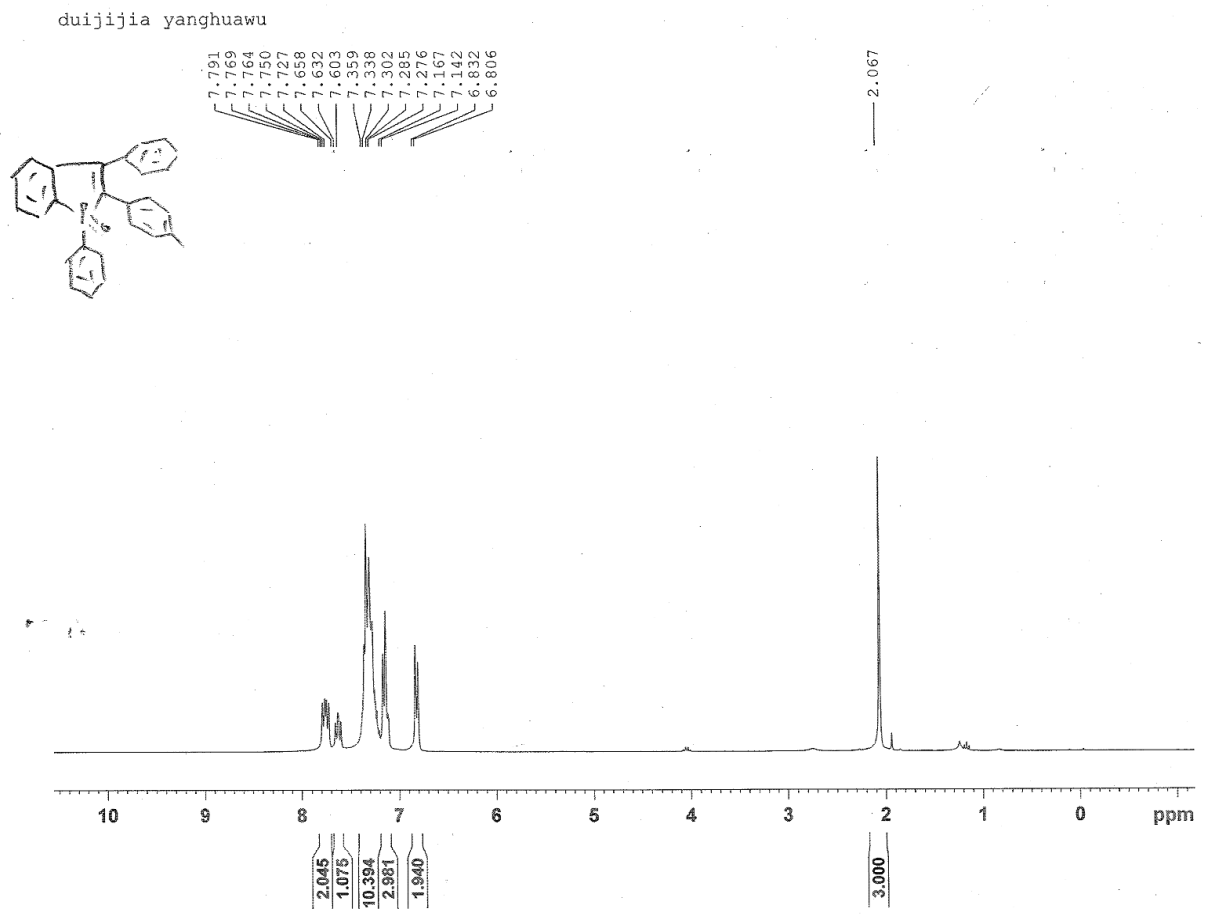

\section{3b ${ }^{1} \mathrm{H}$ NMR}

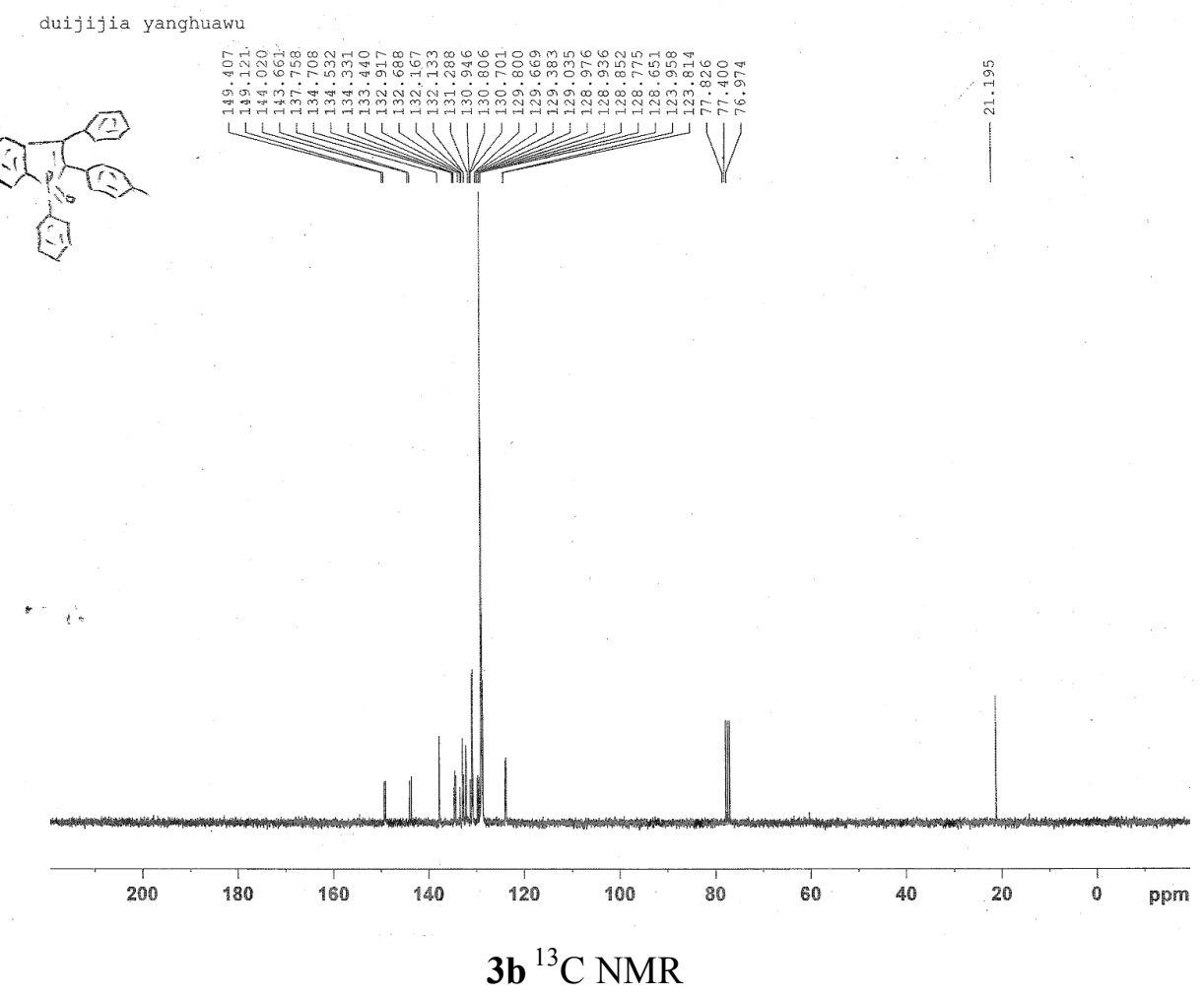



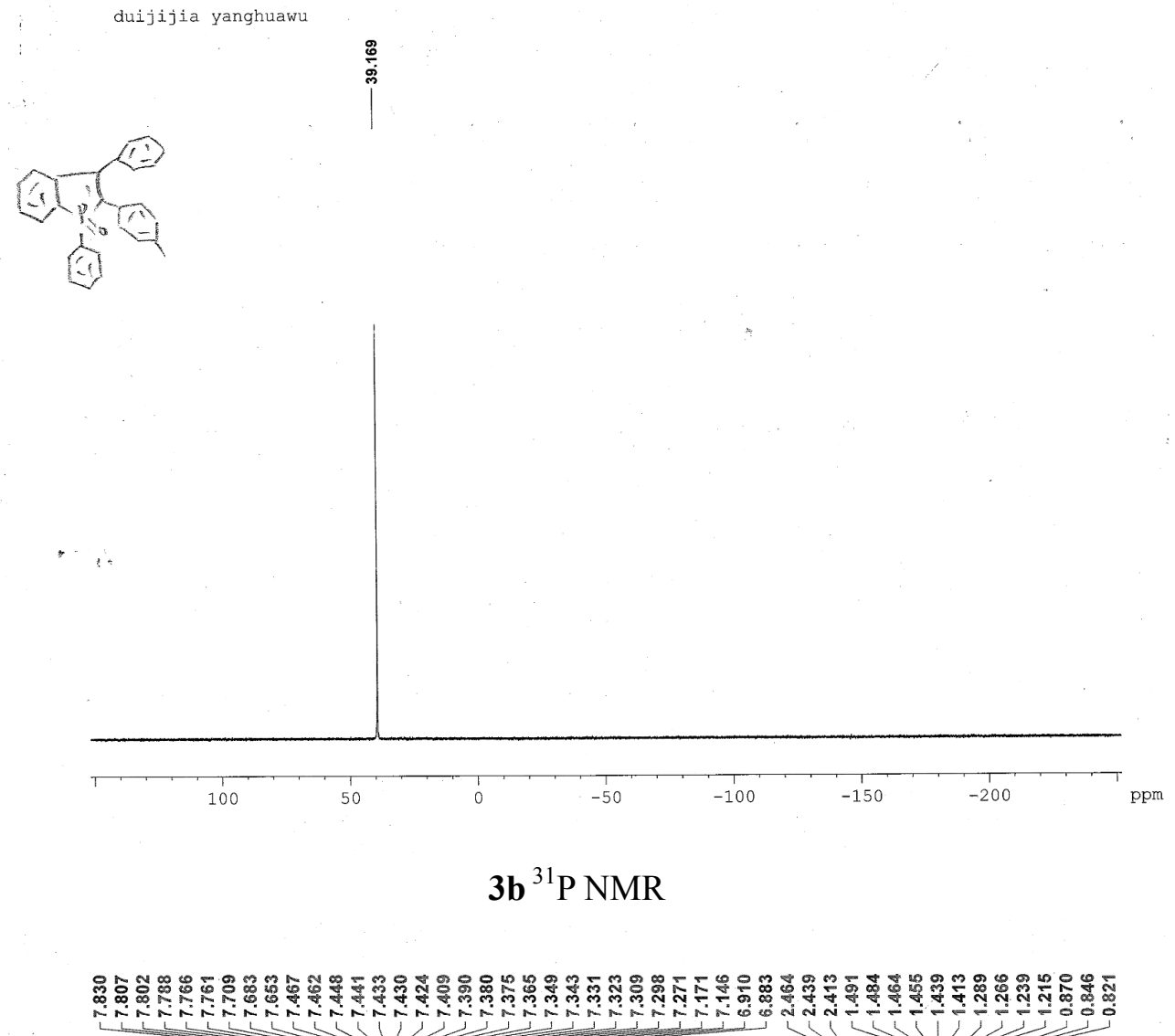

subo HI zhengdingji

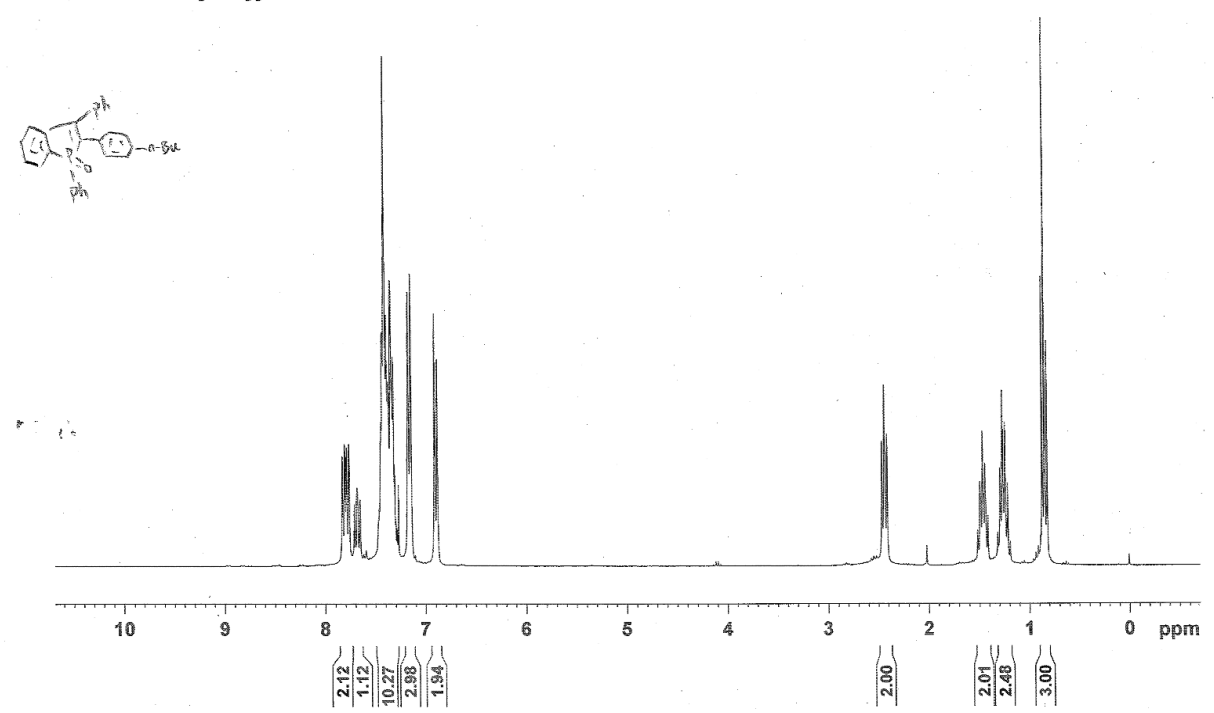

$3 c^{1} \mathrm{H}$ NMR 


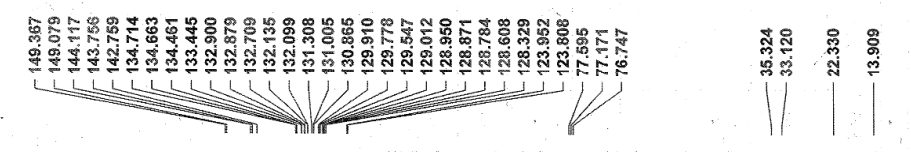

C13CPD dingji

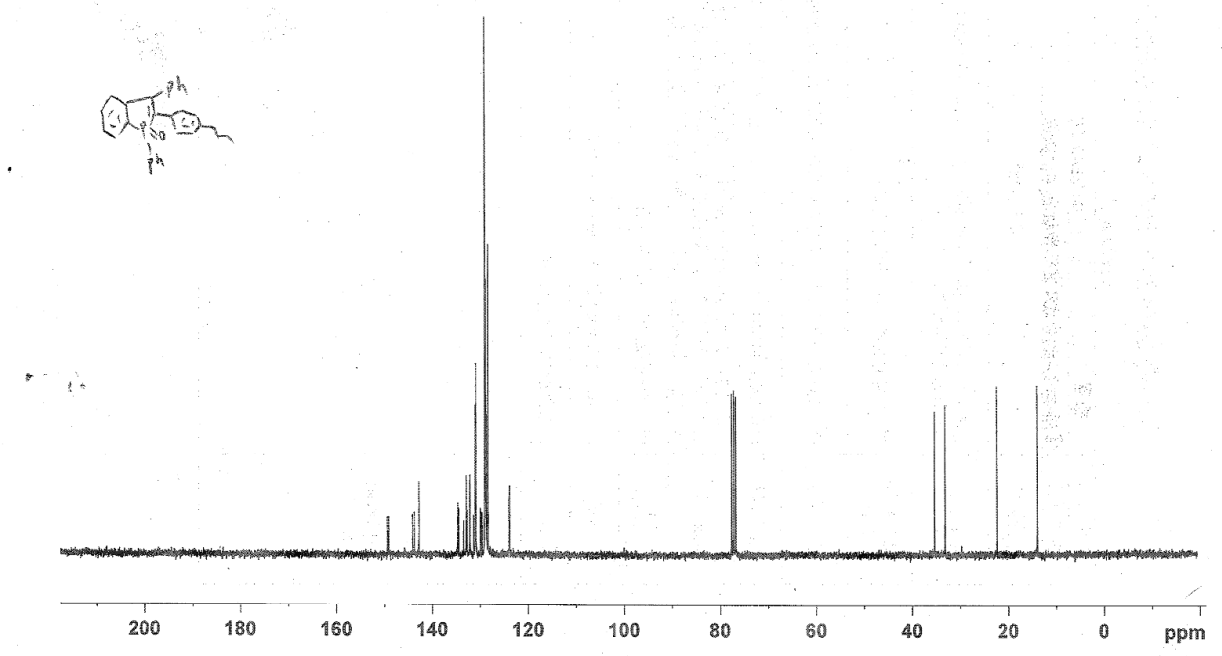

\section{3c ${ }^{13} \mathrm{C}$ NMR}

subo P31 zhengdingji qudaiji कूष

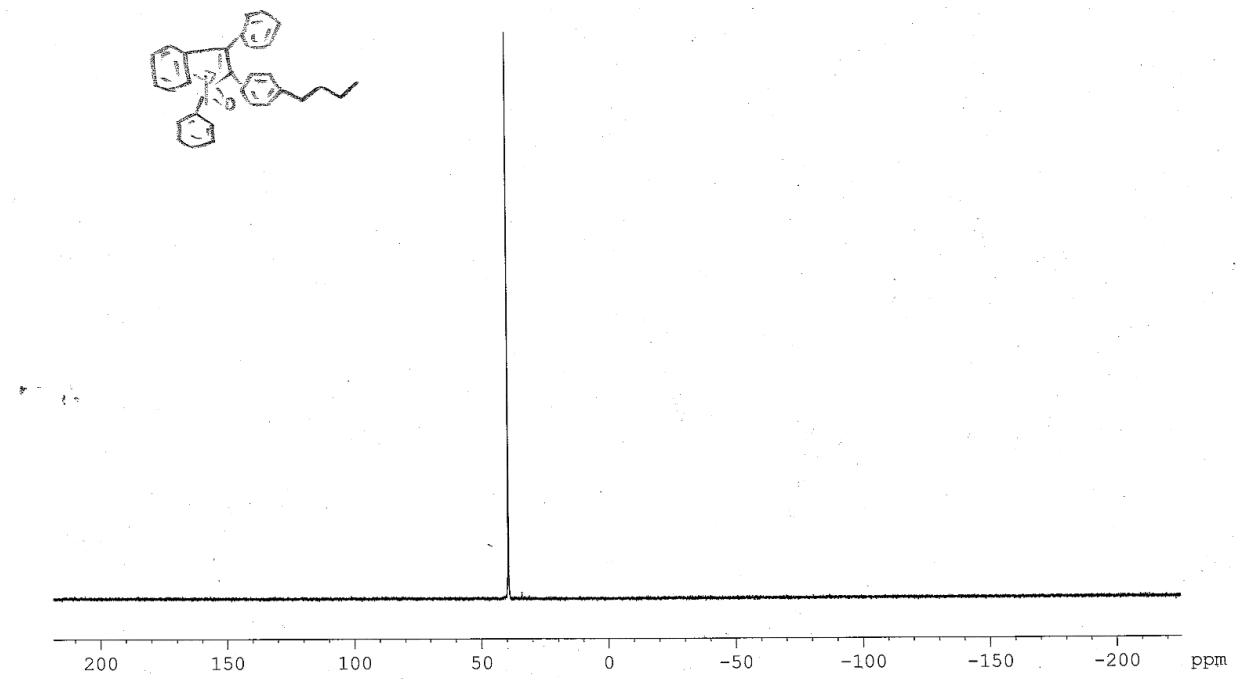

\section{$3 c^{31}$ P NMR}




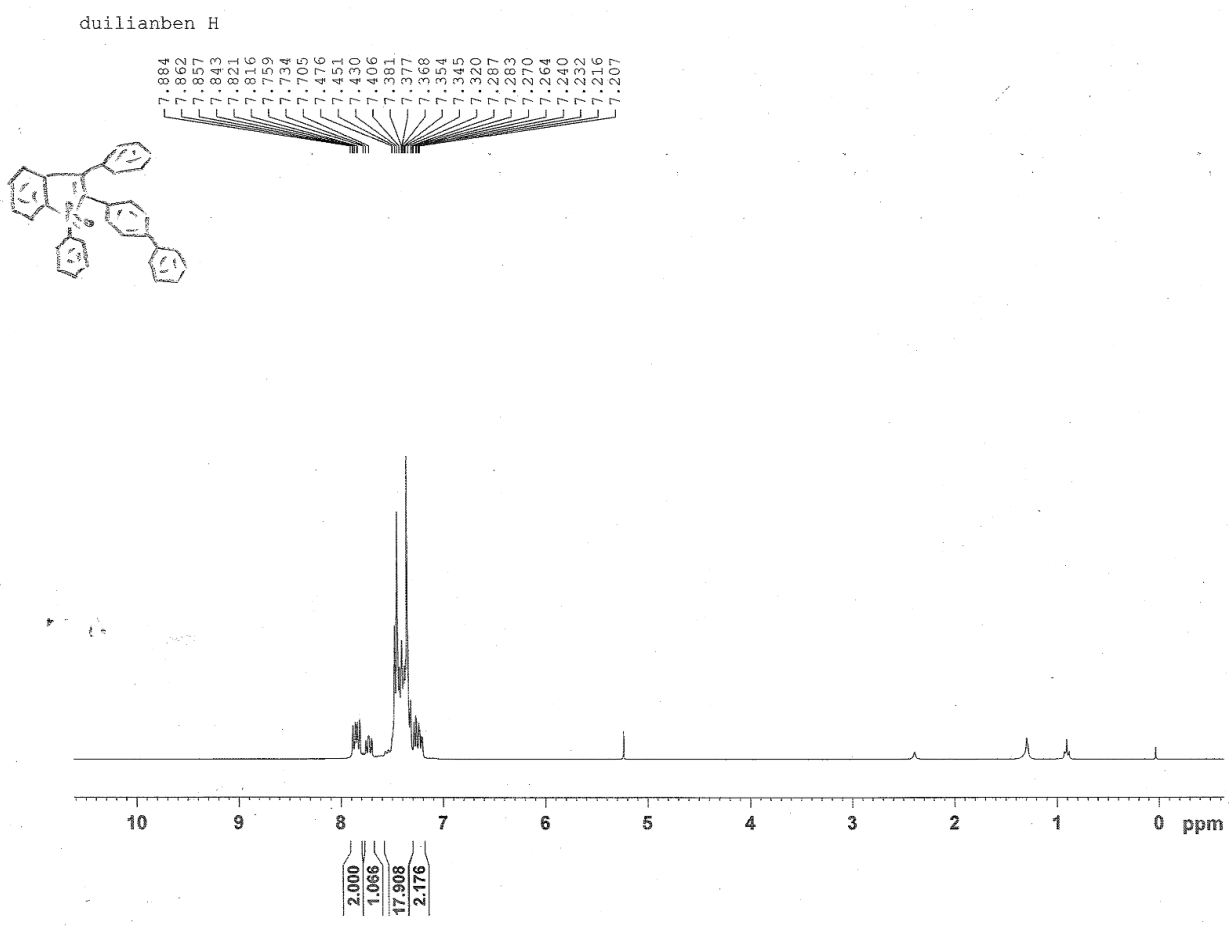

3d ${ }^{1} \mathrm{H}$ NMR

duilianben C13CPD

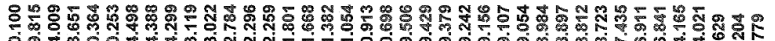

5.7\%

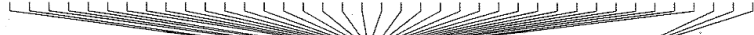

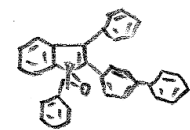

:

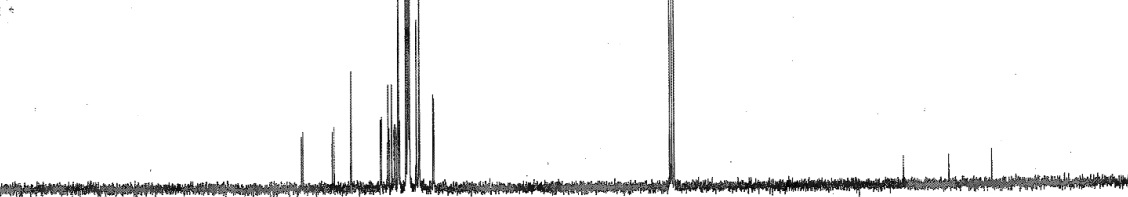

$\begin{array}{lllllllllllllllllllllll}210 & 200 & 190 & 180 & 170 & 160 & 150 & 140 & 130 & 120 & 110 & 100 & 90 & 80 & 70 & 60 & 50 & 40 & 30 & 20 & 10 & 0 & 0\end{array}$

3d ${ }^{13} \mathrm{C}$ NMR 

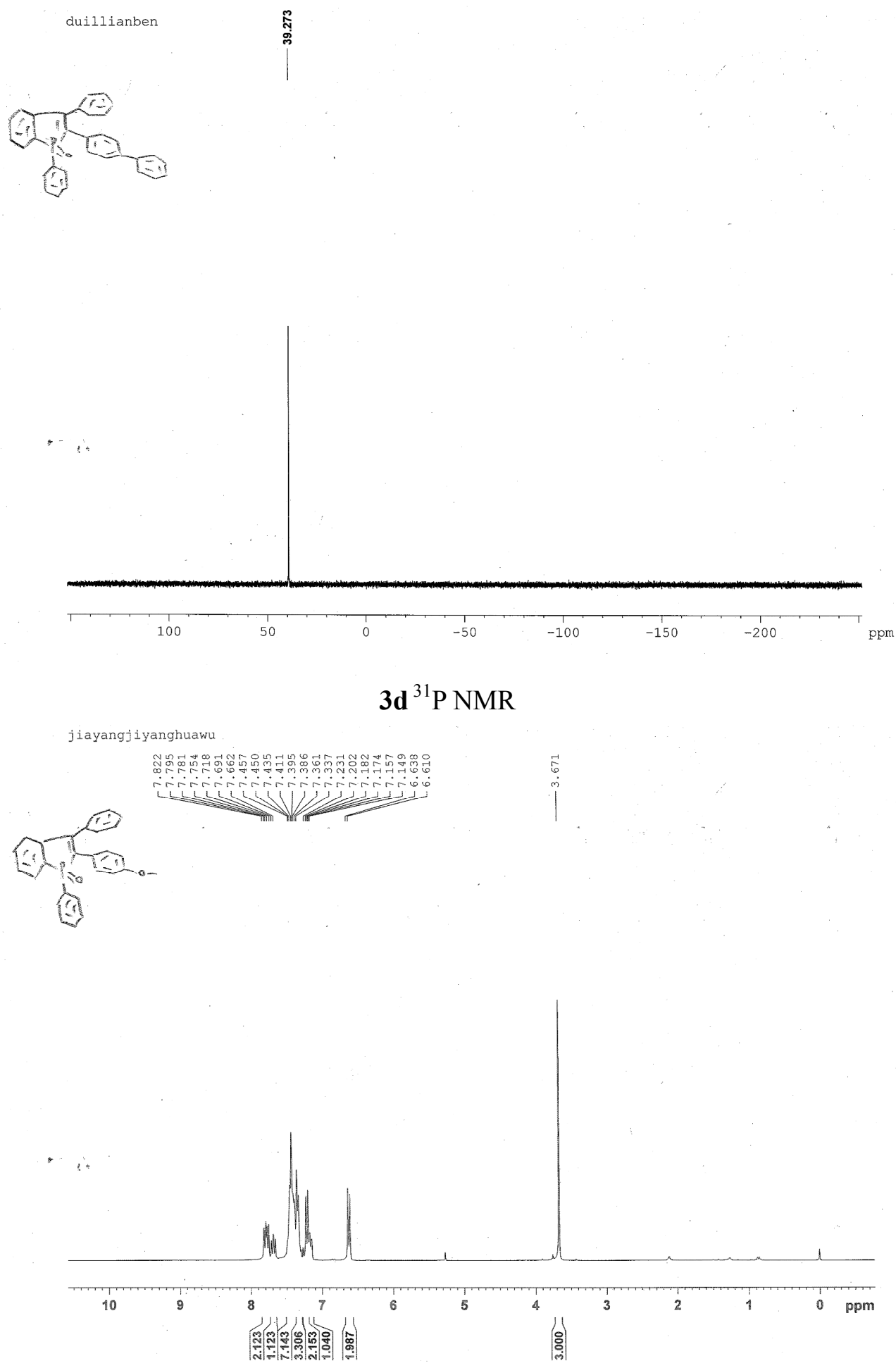

3e ${ }^{1} \mathrm{H}$ NMR 


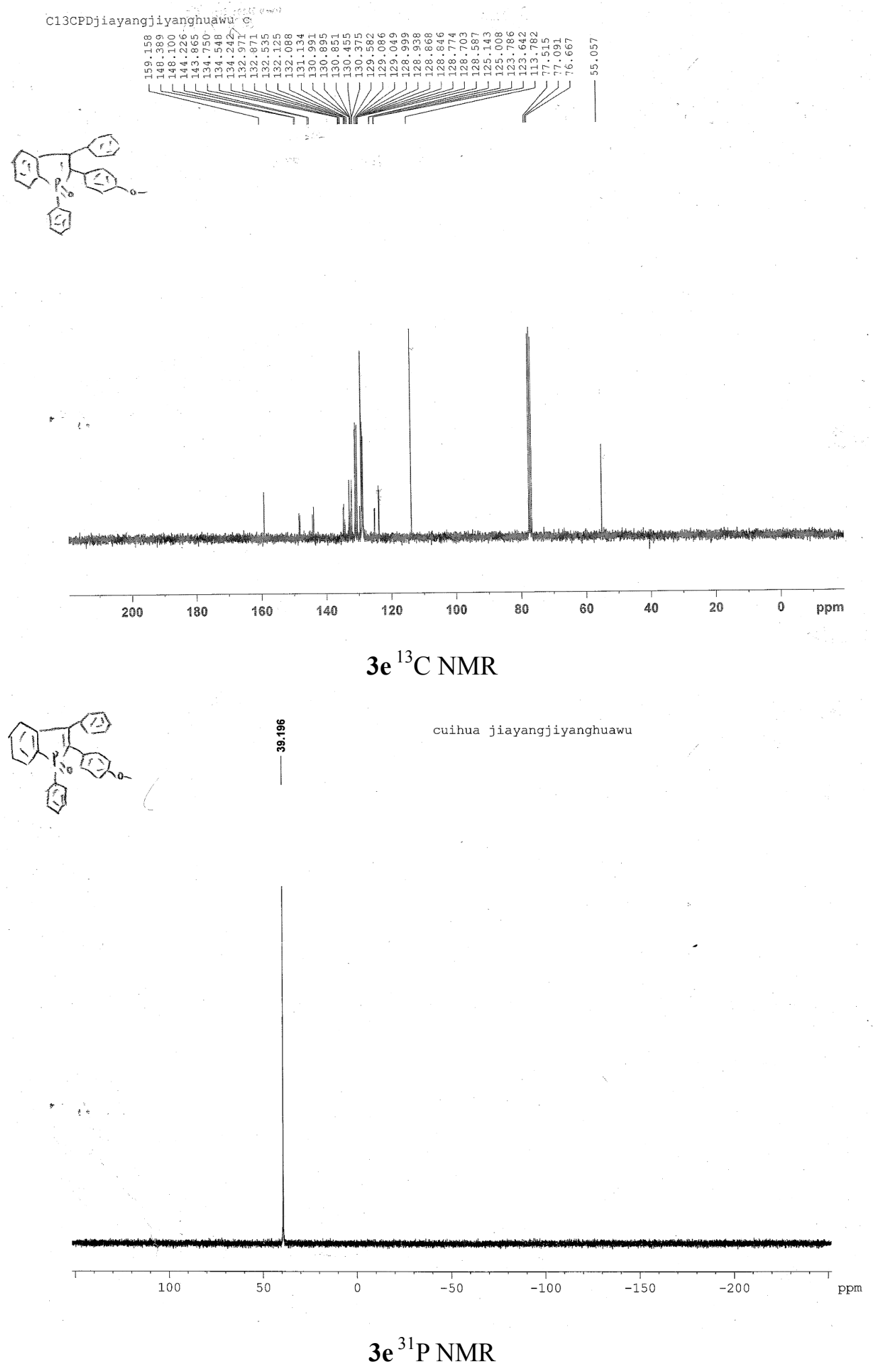

S-31 


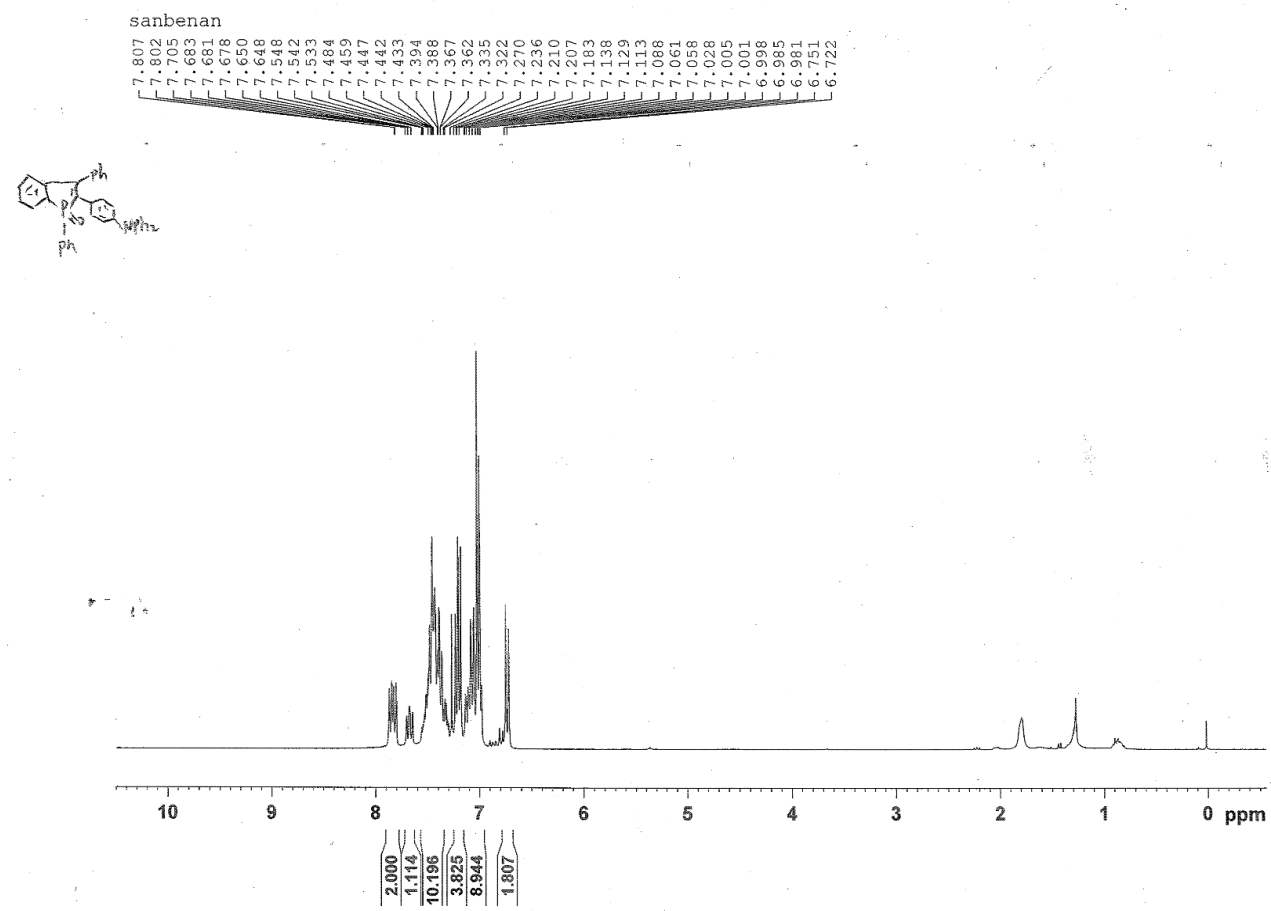

3f $^{1} \mathrm{H}$ NMR

sanbenan

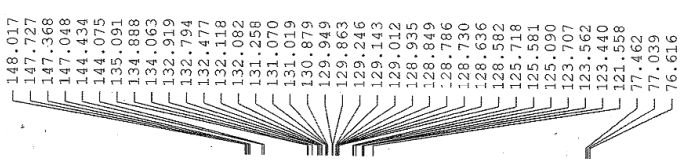

负险ph

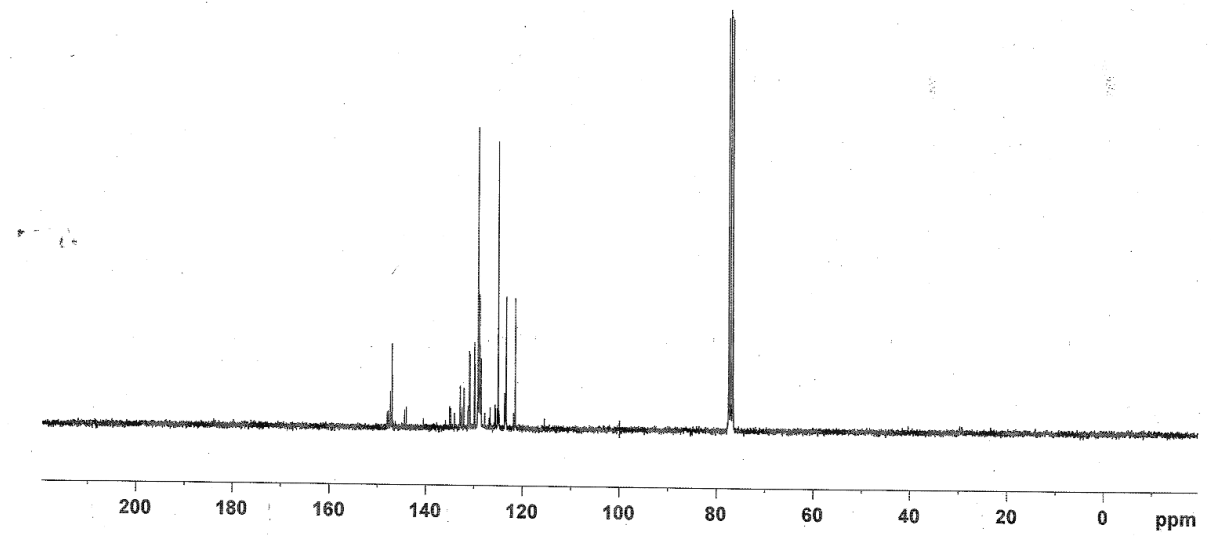

$\mathbf{3 f}^{13} \mathrm{C}$ NMR 
sanbenan chanpin

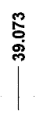

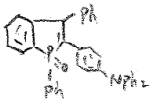
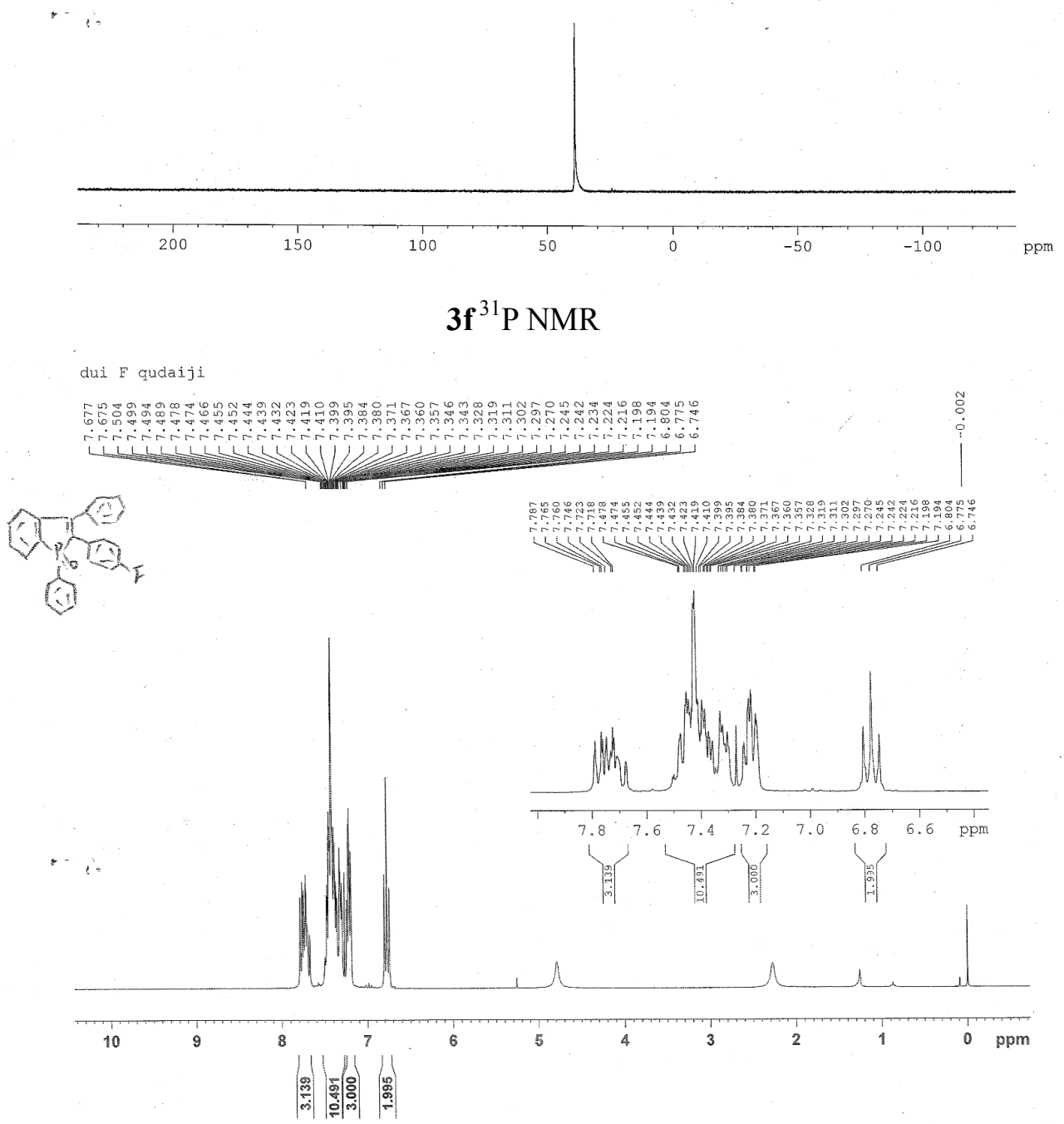

$3{ }^{1} \mathrm{H}$ NMR

S-33 


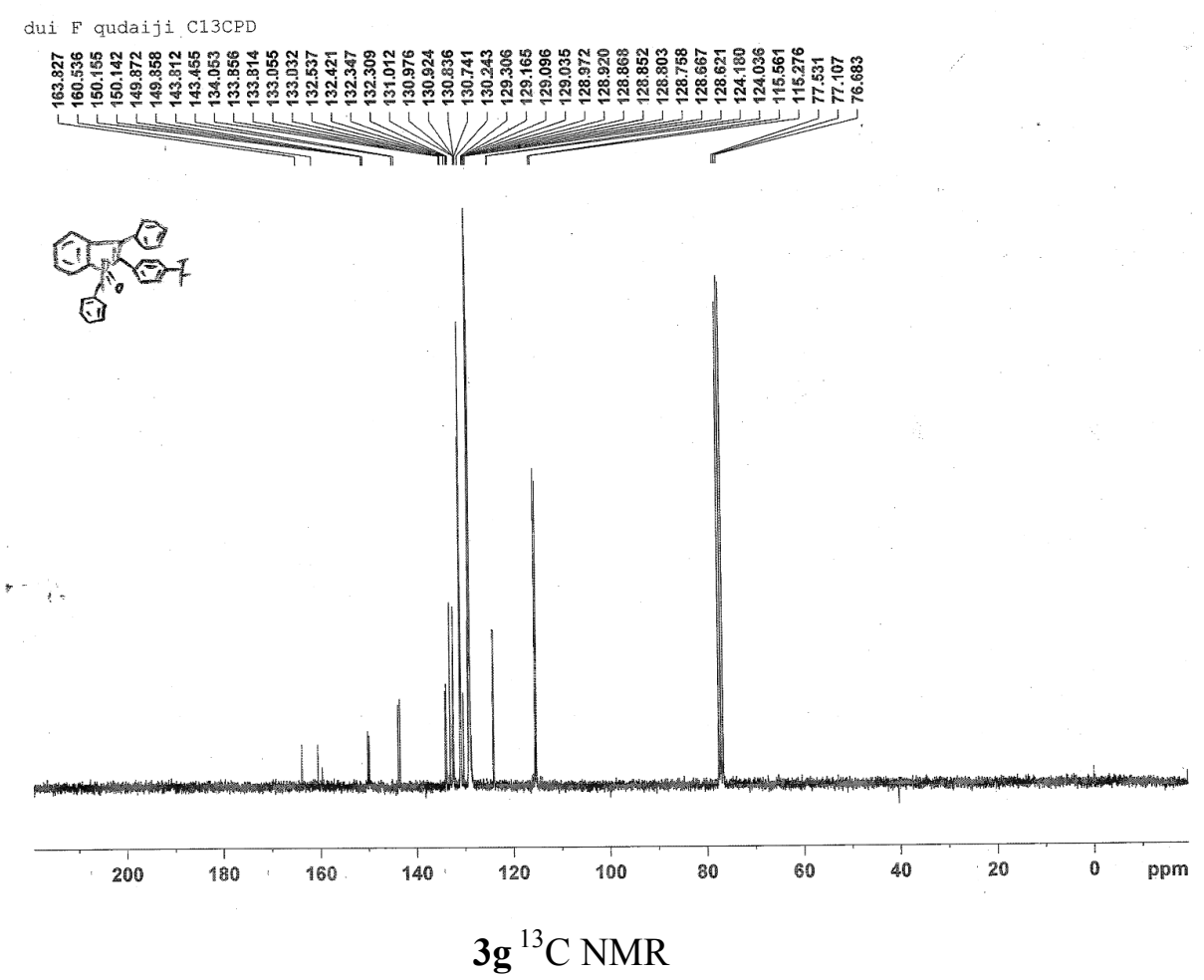

dui $F$ qudaiji
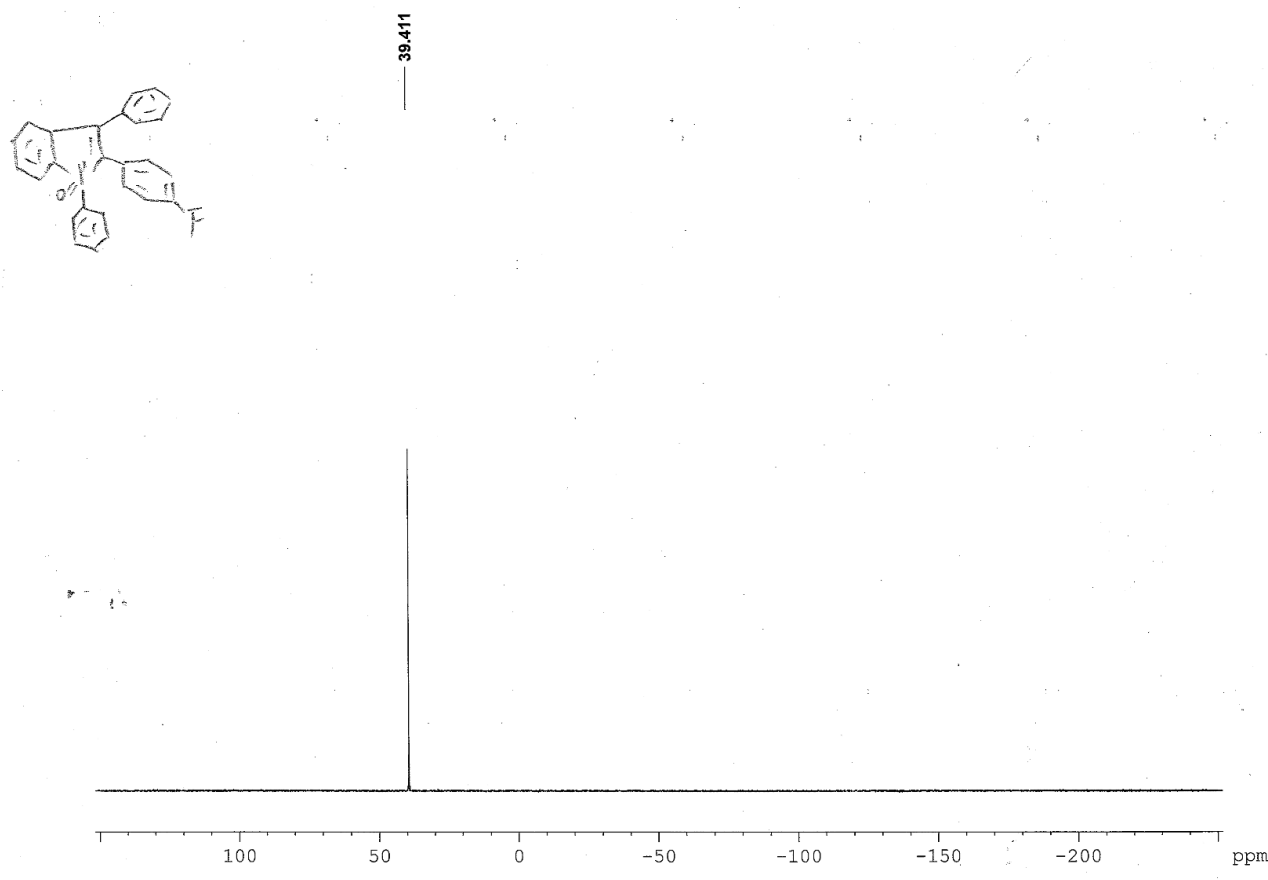

$3 g^{31}$ P NMR

S-34 


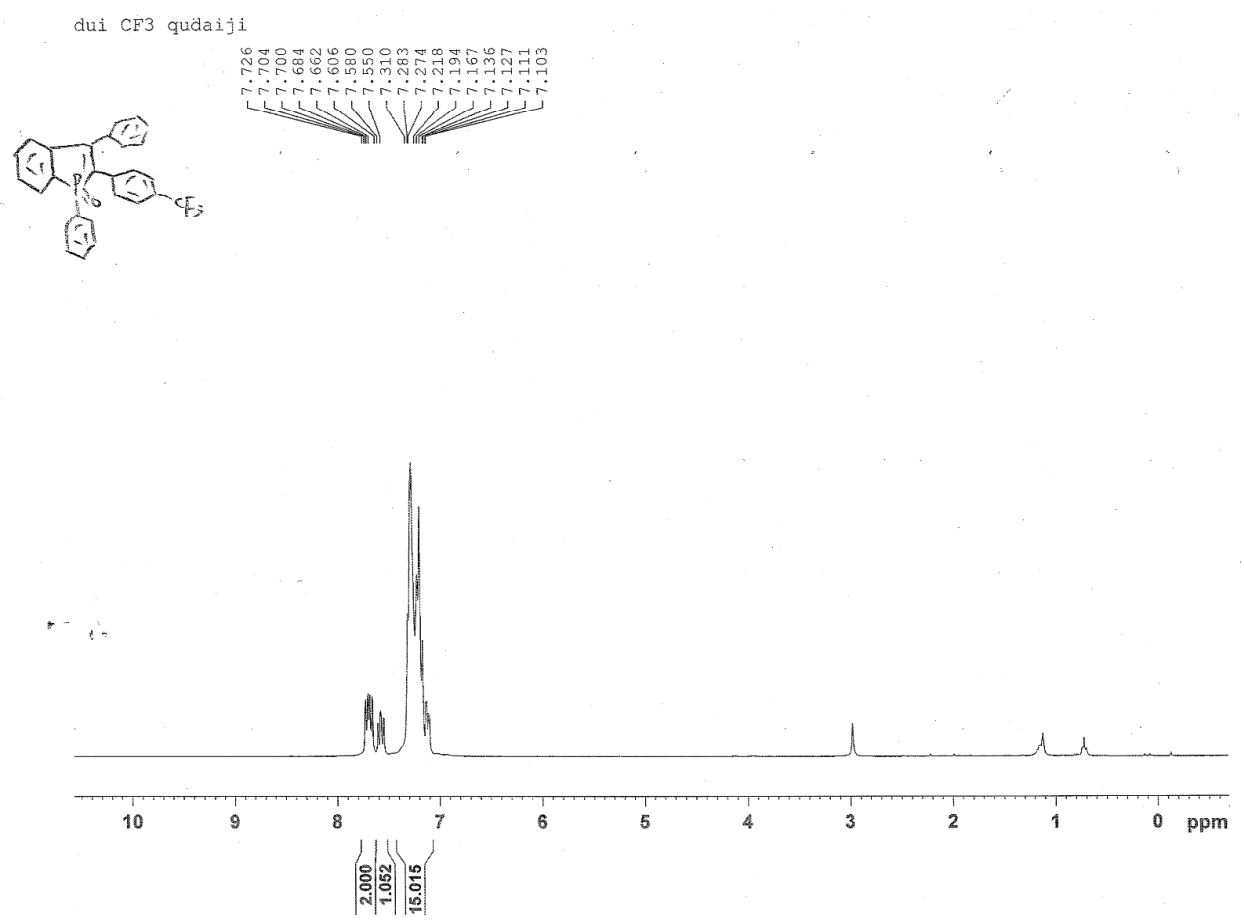

3h ${ }^{1} \mathrm{H}$ NMR
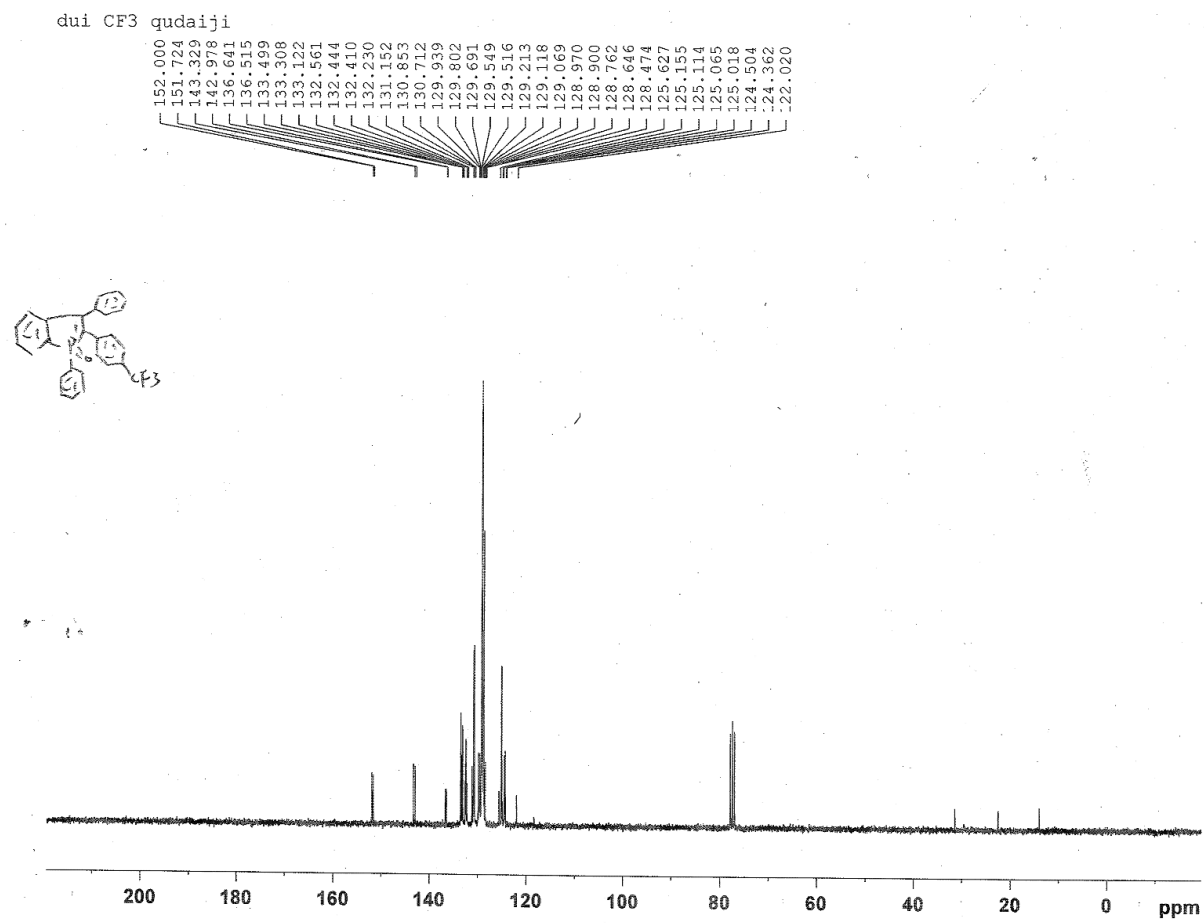

3h ${ }^{13} \mathrm{C}$ NMR 

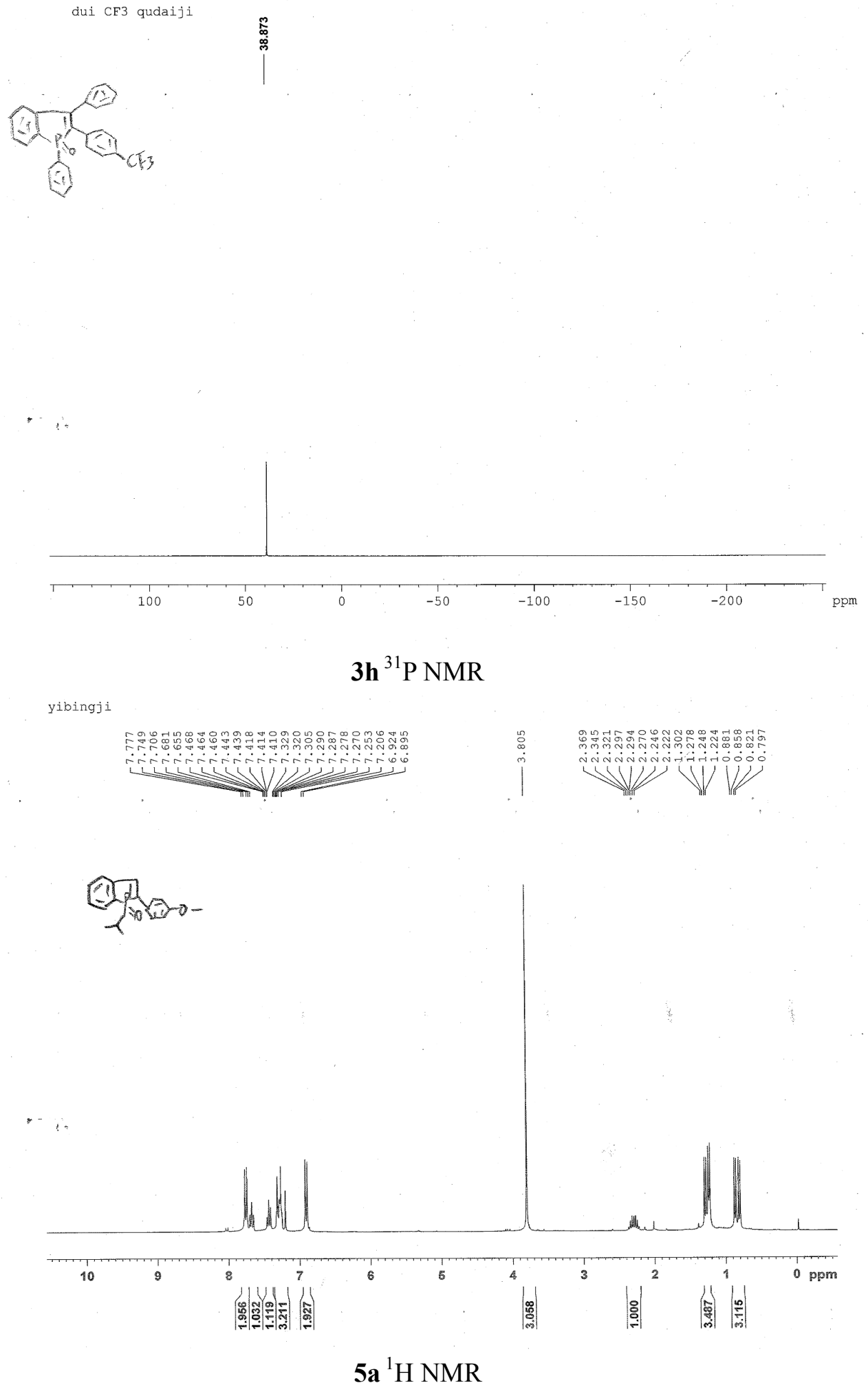

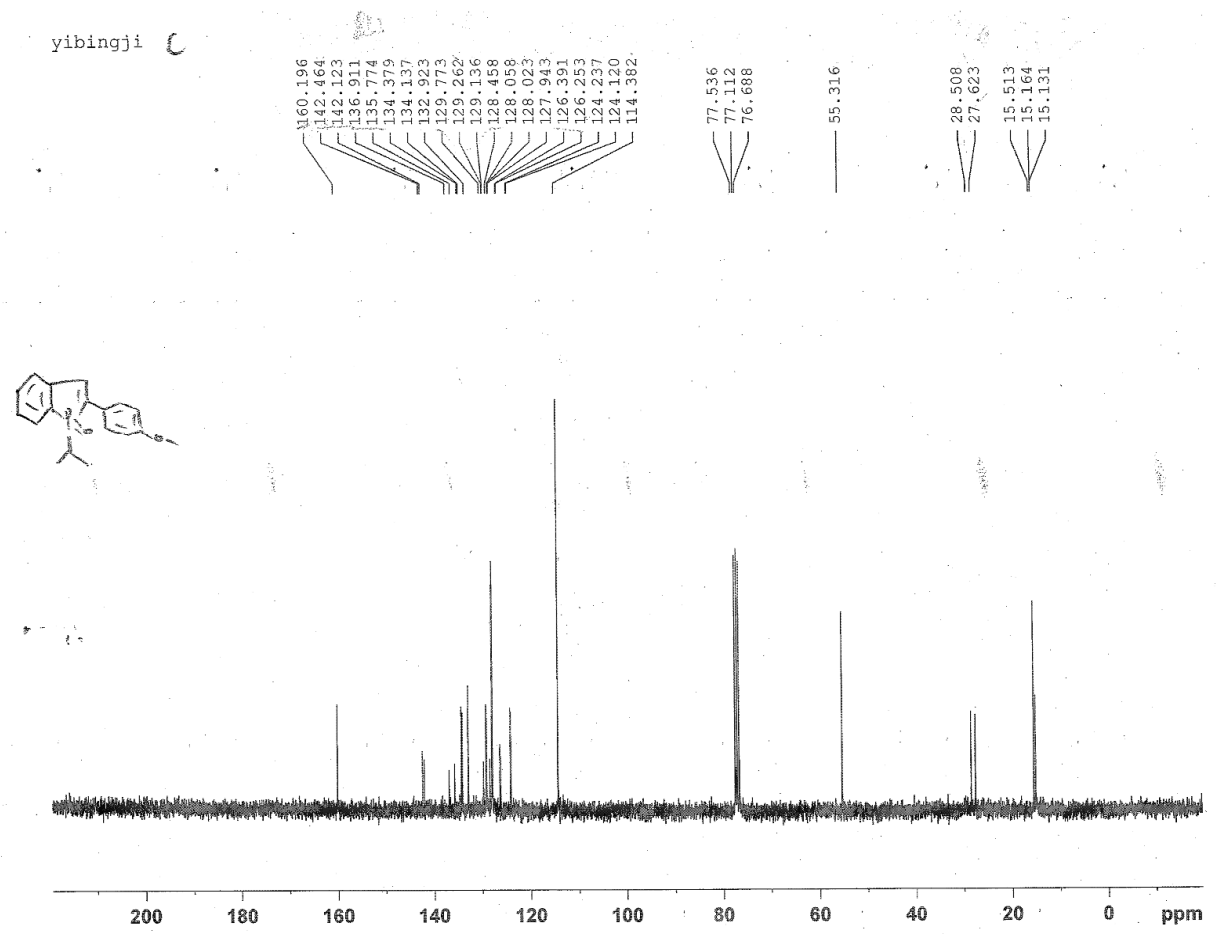

5a ${ }^{13}$ C NMR

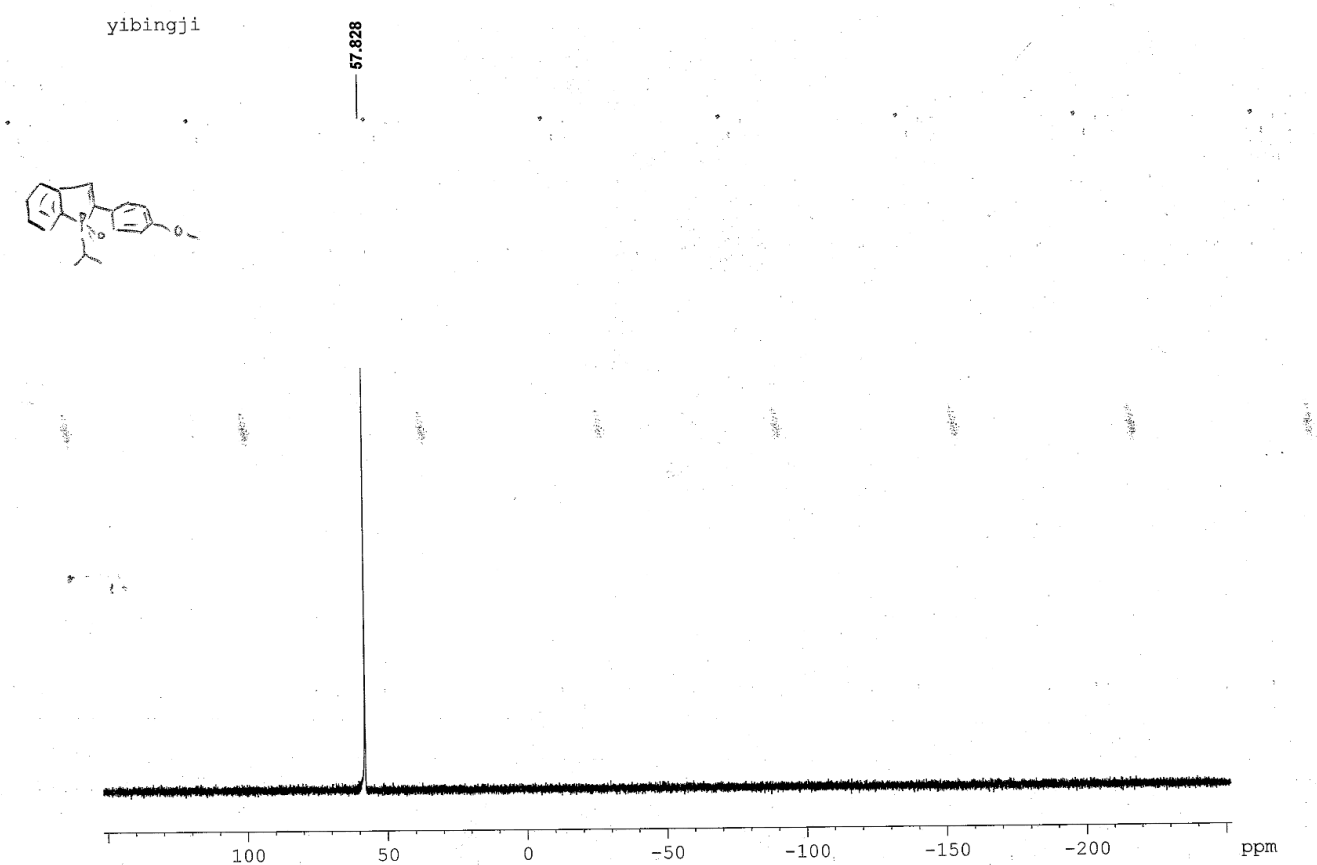

$\mathbf{5 a}^{31} \mathrm{P}$ NMR 


$$
\text { eryian chanpin }
$$

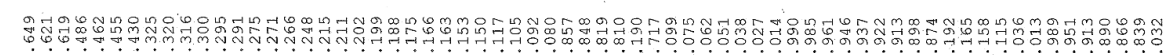

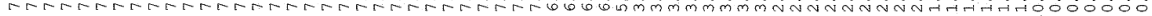

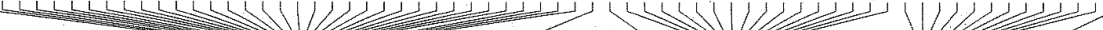
तो जो

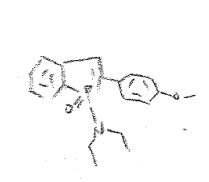

$\cdots$

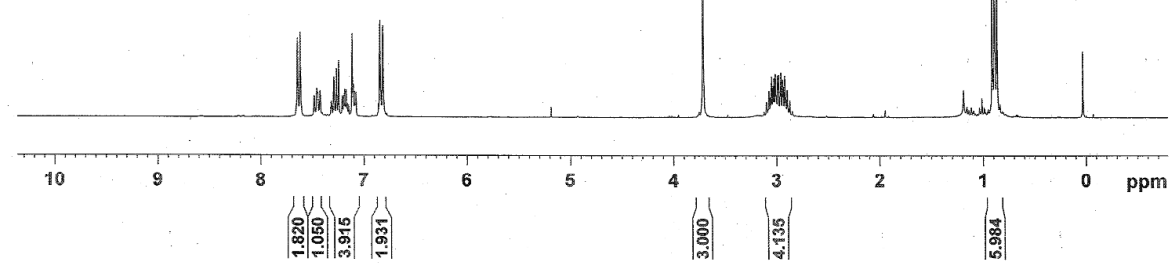

\section{5b ${ }^{1} \mathrm{H}$ NMR}
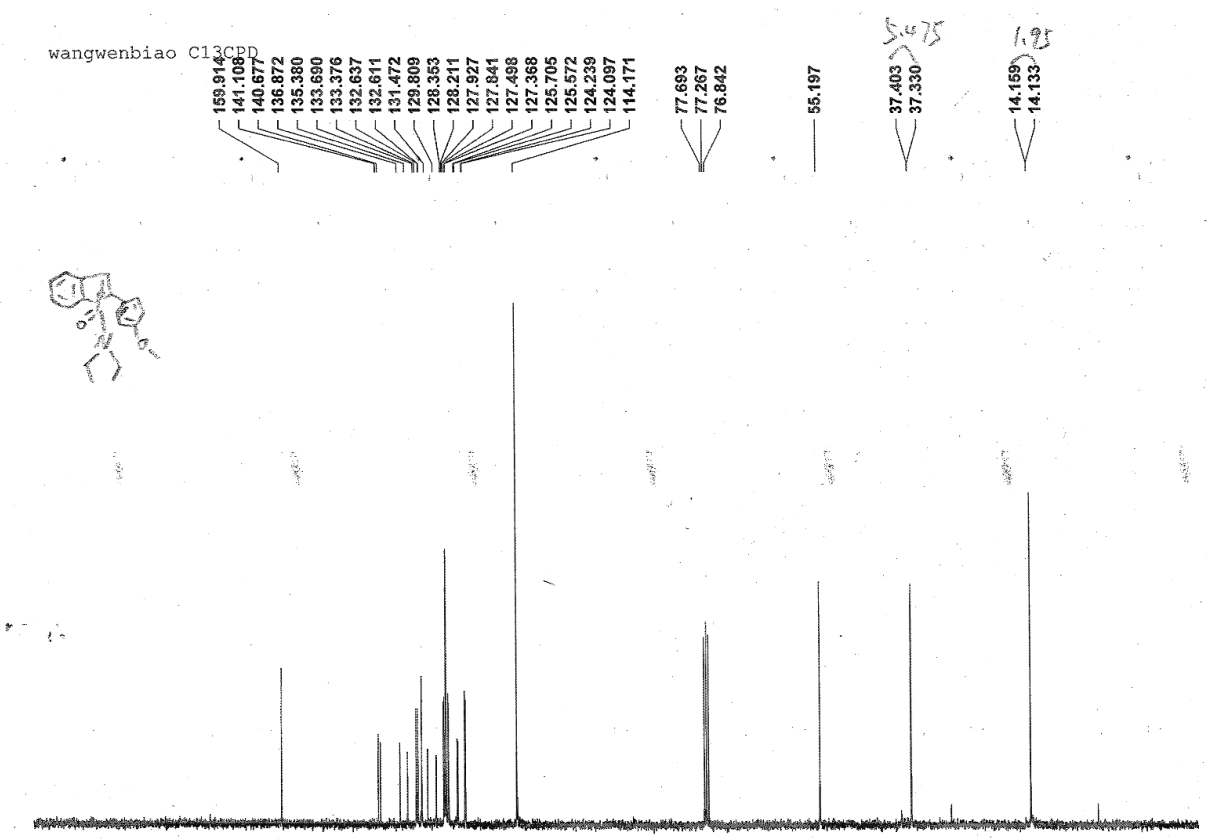

$\begin{array}{llllllllllllllllllllll}200 & 190 & 180 & 170 & 160 & 150 & 140 & 130 & 120 & 110 & 100 & 90 & 80 & 70 & 60 & 50 & 40 & 30 & 20 & 10 & 0 & \mathrm{ppm}\end{array}$

\section{5b ${ }^{13} \mathrm{C}$ NMR}




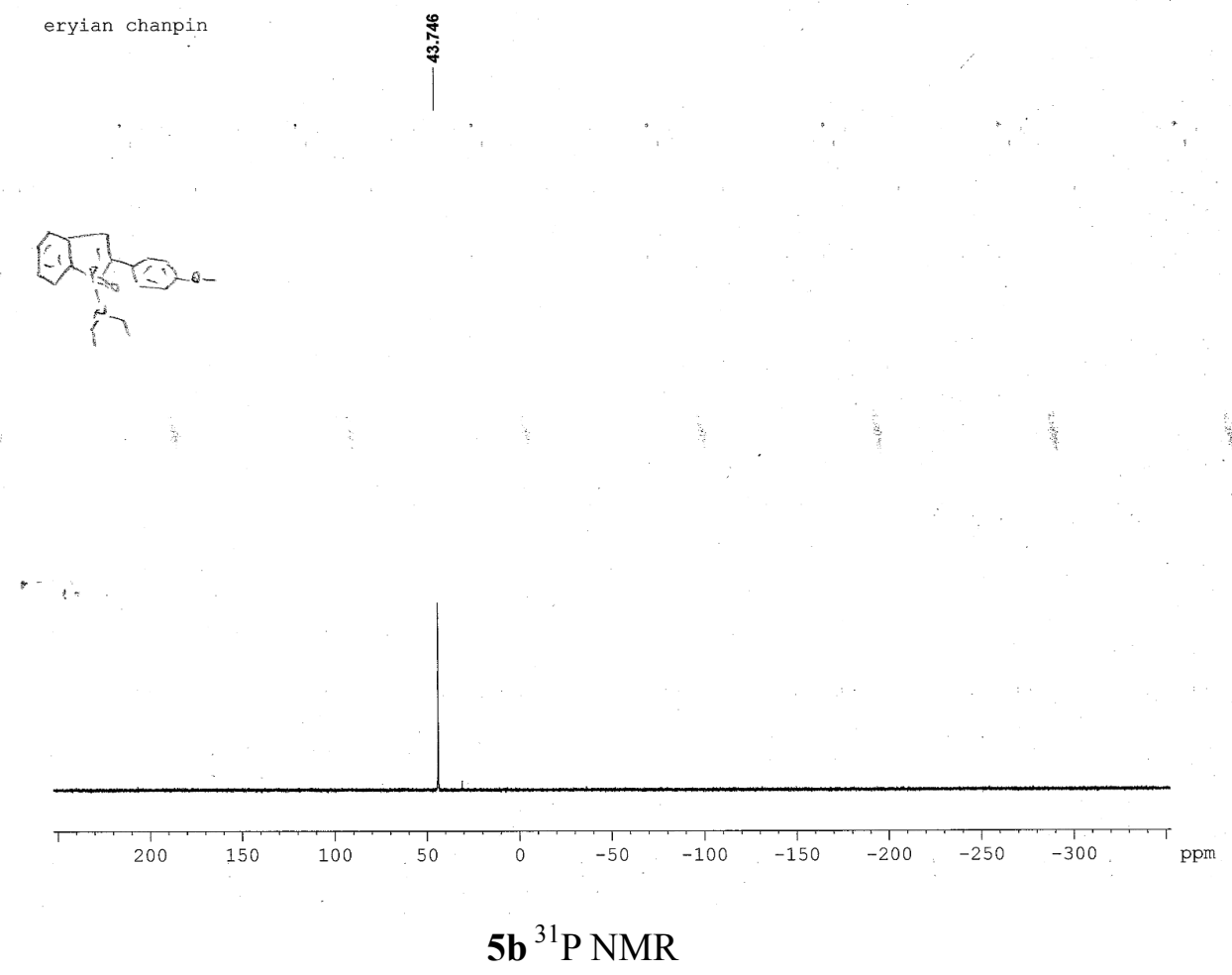

